\title{
WestVirginiaUniversity
}

THE RESEARCH REPOSITORY @ WVU

Graduate Theses, Dissertations, and Problem Reports

2010

\section{Selective placement of actin filaments on protein patterned surfaces}

Lenin J. Leon

West Virginia University

Follow this and additional works at: https://researchrepository.wvu.edu/etd

\section{Recommended Citation}

Leon, Lenin J., "Selective placement of actin filaments on protein patterned surfaces" (2010). Graduate Theses, Dissertations, and Problem Reports. 3019.

https://researchrepository.wvu.edu/etd/3019

This Thesis is protected by copyright and/or related rights. It has been brought to you by the The Research Repository @ WVU with permission from the rights-holder(s). You are free to use this Thesis in any way that is permitted by the copyright and related rights legislation that applies to your use. For other uses you must obtain permission from the rights-holder(s) directly, unless additional rights are indicated by a Creative Commons license in the record and/ or on the work itself. This Thesis has been accepted for inclusion in WVU Graduate Theses, Dissertations, and Problem Reports collection by an authorized administrator of The Research Repository @ WVU. For more information, please contact researchrepository@mail.wvu.edu. 


\title{
Selective Placement of Actin Filaments on Protein Patterned Surfaces
}

\author{
by \\ Lenin J. Leon
}

Thesis submitted to the College of Engineering and Mineral Resources at West Virginia University in partial fulfillment of the requirements for the degree of

\author{
Master of Science \\ In \\ Electrical Engineering
}

Professor Parviz Famouri, PhD., Chair

Professor R. Lloyd Carroll, PhD (Chemistry)

Professor Lawrence Hornak PhD

Lane Department of Computer Science and Electrical Engineering

\author{
Morgantown, West Virginia \\ 2010
}

Keywords: Patterning, Actin, Streptavidin, Biotin 


\section{ABSTRACT \\ Selective Placement of Actin Filaments on Protein Patterned Surfaces \\ by}

\section{Lenin J. Leon}

Motors proteins are used by living organisms to convert chemical energy into mechanical energy. The human body uses such motors proteins to transport materials through cells and, in the case of the biomolecular motor system of actin and myosin, to contract muscle. By understanding how these biological motors work, artificial motors with improved function may be possible and may be engineered to work in complex biological and non-biological environments. Recent research efforts have focused on understanding how to harness the power of, and manipulate the functioning of biological motors for integration into useful nanoscale systems. One important step towards this integration is the binding of motor proteins onto substrates and the full characterization of the system. The aim of this thesis was to study the feasibility of selective immobilization of actin filament motor protein based on the bioaffinity reaction between patterned streptavidin on a substrate and biotinylated actin filaments on an aminopropyltriethoxysilane (APTES)-functionalized glass surface. Gelsolin was used to cap the barbed/positive end of actin and to link actin to biotin molecules on the functionalized surface. Results demonstrate significant binding of actin filaments on streptavidin patterned surfaces via bioaffinity immobilization. Fluorescent microscopy and image processing software were used to characterize these results. Characterization of the APTES-functionalized surface was conducted using atomic force microscopy (AFM). The relationship between actin and gelsolin capping protein was examined as well as non-specific binding control of actin filaments. 


\section{DEDICATION}

I would like to dedicate this thesis to my wife Rachel, for her unconditional support and love, to my son Saul who is the most precious gift given to me, and finally to mi madre querida. 


\section{ACKNOWLEDGMENTS}

I would like to express my sincere gratitude to all the people who made this work possible, especially to my advisor Dr. Parviz Famouri for his advice and for giving me the opportunity to work in his research lab. Also, I would like to express my appreciation to the two other members of my committee Dr. Lloyd Carroll and Dr. Lawrence Hornak and also Dr. Lisa Holland for their suggestions and invaluable technical support.

I want to extend my gratitude to the people in our research lab, Mr. Yongkuk Lee for his help and for providing some of the key chemical elements needed in this research, to Dr. Mingyuan Wei and Mr. Christian White for their technical advice. Finally, I want to thank Dr. Kolin Brown for his help and technical assistance with the equipment in the WVU shared facilities. 


\section{CONTENTS}

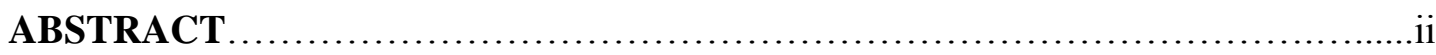

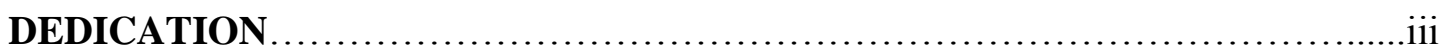

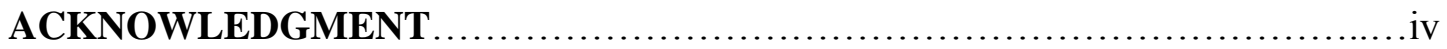

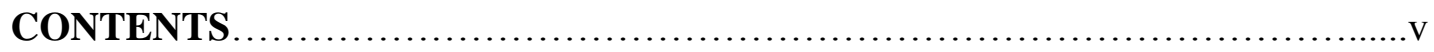

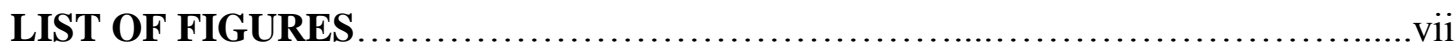

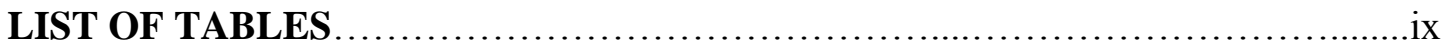

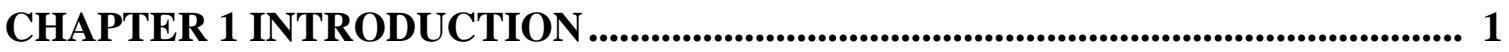

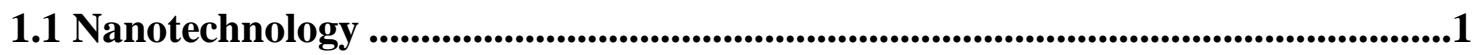

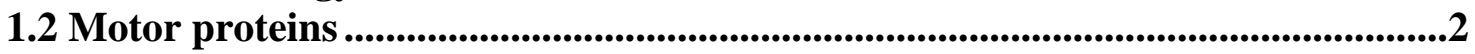

1.3 Biotin and Streptavidin system ..............................................................................3

1.4 Proposed work and motivation ...................................................................................4

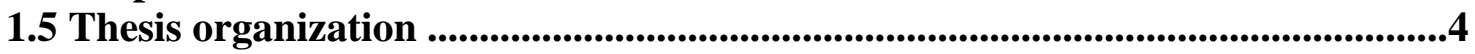

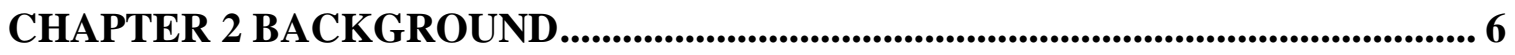

2.1 Types of Protein Immobilization .............................................................................6

2.1.1 Physical Adsorption ....................................................................... 7

2.1.2 Covalent Binding Immobilization........................................................ 8

2.1.3 Bioaffinity Immobilization ................................................................. 9

2.2 Methods of Protein Immobilization ................................................................10

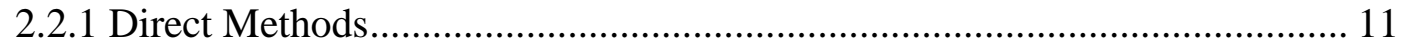

2.2.2 Indirect Methods ..................................................................................... 15

2.3 Actin Motor Protein Structure and Functionality ..............................................19

2.4 Biotin-Streptavidin Structures and Functionality ...............................................22

2.3 Gelsolin Regulatory Capping Protein ......................................................................24

CHAPTER 3 METHODS AND PROTOCOLS .................................................... 27

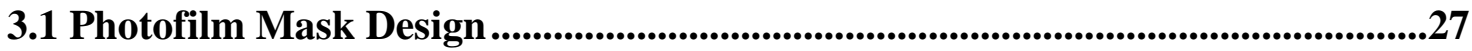

3.2 Photochemical Immobilization Process....................................................................28

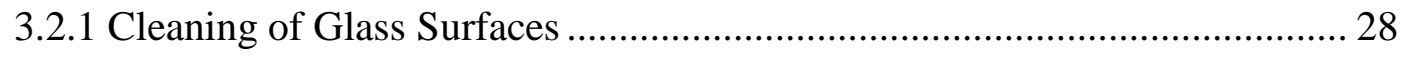

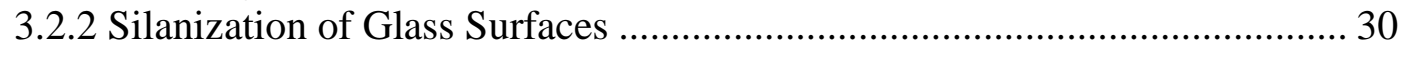

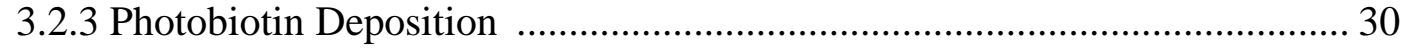

3.3 Microcontact Immobilization of proteins ...........................................................32

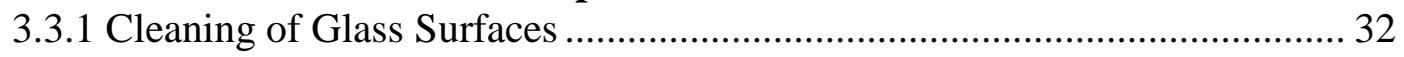

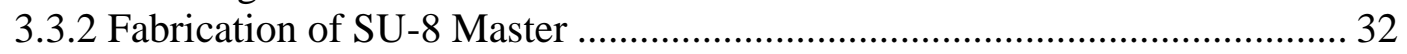

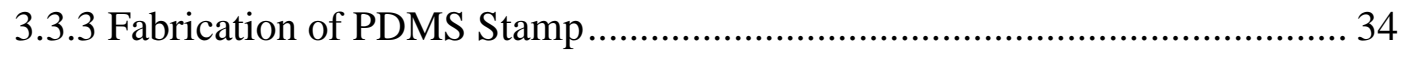

3.3.4Transferring of Protein from PDMS to Glass Substrate ............................. 35 
3.4 Laying Actin Filaments on Protein Patterned Surfaces ...........................................37

3.4.1 Actin Filament Length vs Gelsolin Concentration ......................................... 37

3.4.2 Binding of Actin Filaments in Streptavidin Patterned Surfaces ....................... 38

CHAPTER 4 EXPERIMENTAL SEQUENTIAL DIAGRAMS................................... 39

4.1 Functionalization of Glass Slides with Amino-Silane Groups .............................39

4.2 Immobilization of Photobiotin ....................................................................................40

4.3 Immobilization of Fluorescent Streptavidin...........................................................41

4.4 Conjugation of Actin with Biotinylated Gelsolin and Selective Immobilization

of Actin .........................................................................................................................................42

CHAPTER 5 EXPERIMENTAL RESULTS .................................................................... 44

5.1 Characterization of APTES Coated Slides .......................................................44

5.2 Patterning of Biotin on Amino-Silane Glass Surfaces............................................ 48

5.3 Fluorescent Intensity-Streptavidin Concentration Correlation ............................52

5.4 Protein Patterning by Microcontact Printing .........................................................54

5.5 Optimization of Actin Length For Attachment to Biotin-Patterned Surfaces 56

5.6 Characterization of Non-Specific Binding of Actin ...............................................59

5.7 Attachment of Actin on Streptavidin Patterned Surfaces .....................................65

CHAPTER 6 CONCLUSION AND FUTURE DIRECTION....................................... 72

REFERENCES.............................................................................................................. 75

APPENDIX A EXPERIMENTAL SETUP .................................................................. 81

APPENDIX B PURIFICATION OF ACTIN _...................................................95 


\section{LIST OF FIGURES}

Figure 2.1 Types of bioaffinity immobilization.................................................... 10

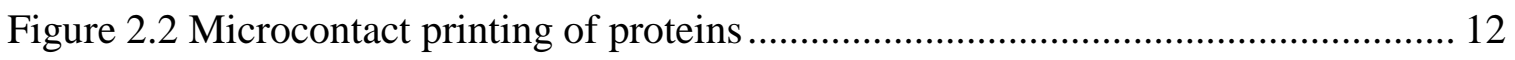

Figure 2.3 Schematic representation of the dip nanotechnology printing technique........ 15

Figure 2.4 Schematic process of patterning of biomolecules using chemical modification of SU-8 photoresist 17

Figure 2.5 Schematic process of patterning of streptavidin using photochemical activation

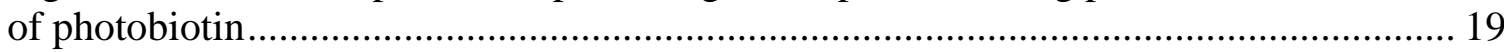

Figure 2.6 Representation of the molecular structure of globular actin ........................ 20

Figure 2.7 Representation of the polymerization of actin filaments .............................. 21

Figure 2.8 Depiction of the molecular structure of streptavidin ................................. 23

Figure 2.9 Process of activation, severing, capping and uncapping of gelsolin .............. 26

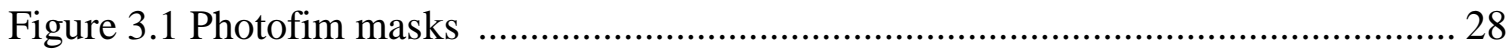

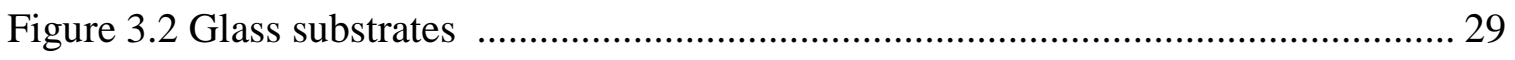

Figure 3.3 Schematic of the flow cell used during experiments .................................. 31

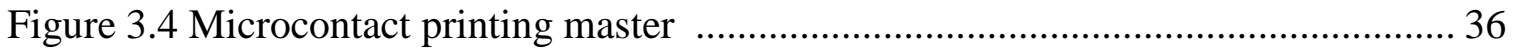

Figure 3.5 Microscope image of fabricated PDMS stamp ........................................... 36

Figure 4.1 Functionalization of slides with amine groups ........................................ 40

Figure 4.2 Immobilization of photobiotin on amine modified surface through activation

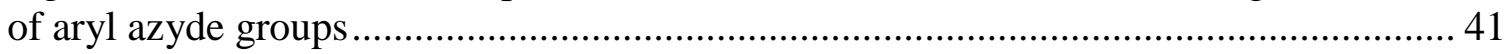

Figure 4.3 Binding of fluorescent streptavidin on patterned photobiotin via bioaffinity

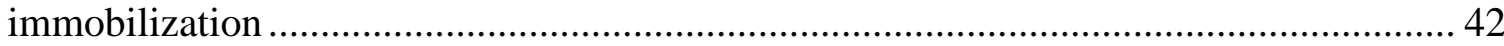

Figure 4.4 Cross sectional view of proposed selective attachment of actin filaments .... 43

Figure 5.1 Contact Mode AFM image of a bare glass surface ..................................... 46

Figure 5.2 AFM images of APTES functionalized glass surfaces ............................... 47

Figure 5.3 Fluorescent characterization of protein patterning ................................... 50

Figure 5.4 Fluorescent images of patterned proteins with distinct geometries................ 51

Figure 5.5 Calculation of pixel intensity values in the region of interest by using Wasabi.

Figure 5.6 Intensity vs. concentration of streptavidin on patterned surfaces................... 53

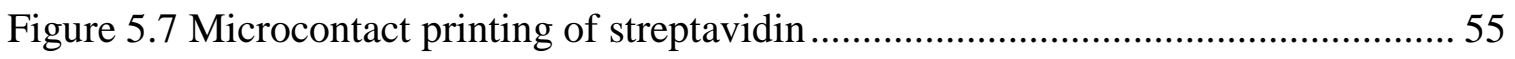

Figure 5.8 Determination of field of view in micron dimensions ................................ 56

Figure 5.9 Actin filaments length calculation at different gelsolin concentrations .......... 58 
Figure 5.10 Relationship between actin filament length and gelsolin concentration ....... 59

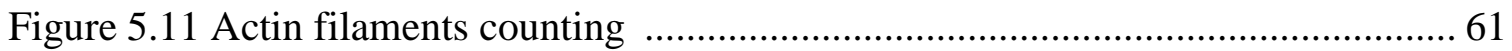

Figure 5.12 Comparison between blocking agents in protein patterned samples .......... 62

Figure 5.13 Non-specific binding blocking agents experimental results ........................ 64

Figure 5.14 Patterning of actin in streptavidin coated surfaces. .................................... 65

Figure 5.15 Determination of actin filaments by direct counting ................................. 68

Figure 5.16 Determination of number of actin filaments after applying pressure driven

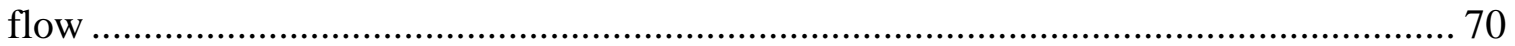

Figure 5.17 Characterization of bound biotinylated gelsolin-capped actin filaments ..... 71 


\section{LIST OF TABLES}

Table 2.1 Functional groups in a protein and the required functional groups in the surface

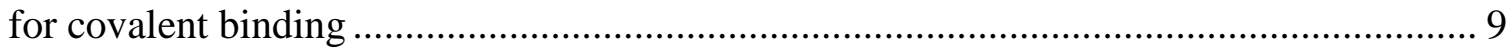

Table 3.1 Parameters used for oxygen plasma treatment of PDMS stamps .................... 35

Table 5.1 Characterization results of APTES functionalization of glass slides with distinct

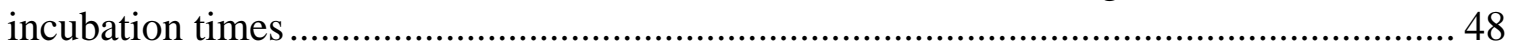




\section{CHAPTER 1. INTRODUCTION}

\subsection{Nanotechnology}

Nanotechnology is a branch of engineering focusing on development of nanosized $\left(10^{-9}\right.$ meters $)$ materials and systems. The vision of this technology was first discussed by Richard Feynman in his famous lecture, "There is plenty of room at the Bottom," given at CALTECH in 1959 [1]. In that lecture, Dr. Feynman mentioned the challenges of manipulating materials at the atomic level, but discussed the endless possibilities of being able to do so. This branch of engineering was first known as "molecular nanotechnology" and was described in Engines of the Creation, by Eric Drexler. Published in 1987, this book served to spread curiosity about the developing field of nanotechnology.

Applications of nanotechnology are numerous, particularly in the areas of medicine and computer science. However, despite recent advancements and the fabrication of innovative nano-sized devices, the field nanotechnology is still in its infancy, with great potential for further developments [2]. Nanotechnology is multidisciplinary, combining aspects of engineering, physics, chemistry, computer science and medicine. Unique properties of materials at the atomic level require knowledge from each of these fields, particularly to create useful systems with those materials in the nanoscale range. Nanoscale systems have widespread potential applications, including being used for disease therapeutics and for development of new, more powerful and space efficient electronic devices. 


\subsection{Motor Proteins}

Motor protein systems are one area of nanotechnology with numerous future applications. Also known as biomolecular motors, these proteins are nanosized motors used in living things to convert chemical energy into mechanical energy. The human body uses such biomolecular motors to transport materials through cells and to contract muscle [3]. By understanding how these biological motors work, artificial motors with improved function may be possible and may be engineered to work in complex biological and non-biological environments. Recent research efforts have focused on understanding how to harness the power of, and manipulate the functioning of biological motors for integration into useful nanoscale systems. A unique property of biomolecular motors is their potential ability to transport a molecular cargo [4], which has potential use for targeted drug delivery.

Actin is a commonly used biomolecular motor system. In living organisms, actin is part of the cellular cytoskeleton and participates in the process of cell motility. This protein plays a key role in defining the shape and mechanical properties of cells [5]. Motion produced by actin is possible due to the interaction with another motor protein known as myosin. Myosin transforms the chemical energy from adenosine triphosphate (ATP) into mechanical movement that allows myosin to "walk" along actin filaments.

Actin filaments are strands of polymerized globular actin to which additional proteins can attach. Tropomyosin and troponin attach to actin filaments and serve to cover the sites on actin molecules where myosin attaches [6].

Assembled actin filaments have a defined polarity. This characteristic is important due to the fact that all but one type of myosin (myosin VI) "walks" towards the positive 
or barbed end of the filament [7]. This characteristic myosin motion is the fundamental concept in the development of actin-based devices that can be powered by the interaction of these two motor proteins. Other biomolecular motor protein systems with similar properties, such as kinesin-microtubule, have been extensively studied. Applications such as transportation of cargo in isopolar arranged microtubules has been demonstrated $[8,9$, 10]. More recently studies have focused on the myosin-actin motor protein pair due to their advantages over kinesin and microtubule motors. Among those advantages is their smaller size, which demonstrates the trend of device miniaturization $[10,11]$. Another important advantage is the flexibility of actin filaments that enables the development of devices with complex geometries.

\subsection{Biotin and Streptavidin System}

Biotin and streptavidin are one of the most widely used ligand-binding systems for research and development of nanoscale devices [12]. These proteins can be used in a wide array of applications due to their uncomplicated chemistry. One important advantage of biotin-streptavidin is the high affinity between the two molecules. The disassociation constant for this bond has been determined to be approximately $10^{15}$, which is the highest (non-covalent binding) disassociation constant known in chemistry [13]. Another important advantage is the fact that streptavidin possesses four binding sites making it particularly suited for the attachment of multiple biotinylated moeities [12]. This system is discussed in detail in Chapter 2. 


\subsection{Proposed Work and Motivation}

The ultimate goal of the research project described here is to create selectively patterned surfaces with immobilized actin. One important task necessary to meet the objectives of the project is to create a bond between motor proteins and glass substrate. In this thesis, we present data to demonstrate this interaction, including fluorescent characterization of actin binding onto protein-coated surfaces. This data was acquired by selectively patterning a biomolecular complex to serve as the linkage between actin motor proteins and the substrate. Biotin and streptavidin served as biomolecular linkages and were chosen for this purpose due to the stable and consistent chemistry required for their surface immobilization. The high specificity of this system ensures a strong and ordered molecular linkage structure for the immobilization of actin motor proteins. The approach used to achieve this interaction involved a photoderivative of biotin, which undergoes structural changes upon UV exposure, and a photofilm mask to selectively irradiate specific areas. The preserved photobiotin was then incubated with fluorescenttagged streptavidin for visualization of the pattern and for linkage with actin conjugated to gelsolin capping protein. Gelsolin not only caps the positive end of actin protein, but also serves to hold biotin molecules that are used to link the filaments to the immobilized streptavidin on the surface via bioaffinity interaction

\subsection{Thesis Organization}

The following thesis will be organized as described below: 
Chapter 2. This chapter will review relevant concepts such as methods for micropatterning of proteins, structure and functionality of the biotin-streptavidin system, structure and functionality of the actin motor protein and the function of gelsolin as capping and length regulatory protein.

Chapter 3. This chapter will explain the methods and procedures used during the experiments.

Chapter 4. This chapter will describe the experiments sequence using diagrams to explains every step of the selective immobilization process.

Chapter 5. This chapter will explain the methods for data interpretation, analysis and discussion of results.

Chapter 6. This chapter will discuss the conclusions made based on the results obtained in Chapter 5 and propose future directions of the research. 


\section{CHAPTER 2 BACKGROUND}

In this chapter fundamental concepts essential to the understanding of studies outlined in this thesis are presented. The chapter begins with a discussion about the different types of protein immobilization, such as physical adsorption, covalent binding, and bioaffinity immobilization. The chapter continues with a description of specific methods developed from types of immobilization followed by a discussion of fundamental theory about motor proteins. The motor protein actin is discussed in detail, including its role in cell motion and transportation. The underlying principles of the interaction of the small vitamin biotin and the protein streptavidin are also described. Finally, the chapter ends with a discussion of the regulation of actin filament length by the capping protein gelsolin.

\subsection{Types of Protein Immobilization}

Protein immobilization consists of the placement of protein in a substrate which depending of the type of immobilization used can allow proteins to keep their functionality [14]. The loss of protein functionality is caused by the random attachment of the protein in the surface, which affects its natural structure. In order to keep their functionality proteins needs to be attached in such ordered way that their original structure can be preserved. The type of protein and the intended place of immobilization are the most important factors that ultimately determine the immobilization strategy to be implemented. 


\subsubsection{Physical Adsorption}

This type of immobilization relies only in the intermolecular forces (i.e van der Waals forces, hydrophobic and polar interactions) between the molecule and the surface in which protein is immobilized. The placement of protein is this type of immobilization is completely random due to the interaction between the molecules and the surface, which in some cases affects or totally inhibits the functionality of the proteins. Despite the simplicity of this method, it is probably the most challenging to understand and model [15]. Initial conditions of the assay have a big impact in the molecule or protein coverage due to changes in surface charges on the surface or net charge distribution in proteins, therefore altering the interaction between molecules and surface [16].

Proteins have high affinity to be physically adsorbed by hydrophobic surfaces. Such interaction has been demonstrated in several investigations, an example is the work done by Yoon and coworkers in 1996. They conducted experiments using carboxyl modified beads and BSA to determine which kind of intermolecular force was more predominant in the physical adsorption of the BSA on the bead surface [17]. They concluded that the adsorption of protein was mainly dominated by hydrophopic interactions and hydrogen bonding. Another example of the physical adsorption in hydrophobic surfaces was shown by Ayhan et al. They concluded that higher levels of protein adsorption occurred when hydrophobicity levels were increased on the surfaces (in their case PMMA surfaces) [18].

This method is perhaps the simplest protein immobilization strategy experimentally but it has several disadvantages among the most important are the fact 
that the protein functionality can be compromised and also the attachment to the surface is rather weak and proteins can be removed by some types of washing buffers.

\subsubsection{Covalent Binding Immobilization}

The covalent binding is the type of immobilization that takes places by the chemical binding between the surface and the protein. This type of binding occurs when one of the chemical groups present in proteins (amine, thiol, carboxyl or hydroxyl) react with a suitable functional group in the surface [18]. The surface where proteins are immobilized has to be functionalized with a certain type of cross-linking agent that would then bind covalently to any of the mentioned functional groups present in proteins.

One of the most used methods to generate covalent bonding is the amine chemistry. In this type of chemistry the surface is functionalized with an element such as Lysine. This amine funcionalized surface is then treated with N-Hydroxysuccinimide (NHS), which is a common crosslinking due to its affinity to amine groups. The protein is then exposed to this surface and by the interaction of the ester group in NHS and the nucleophilic groups present in the protein an amine bond is formed $[14,19]$. Other types of chemistry such as epoxy chemistry offer an easy way to obtain covalent bonds with minimal chemical modification of the protein ensuring the stability of the protein. Table 2.1 shows the most common chemistry groups present in proteins and the required functional groups in the surface to establish a covalent bond. 


\begin{tabular}{|c|c|c|}
\hline side groups & amino acids & surfaces \\
\hline$-\mathrm{NH}_{2}$ & Lys, hydroxyl-Lys & $\begin{array}{l}\text { carboxylic acid } \\
\text { active ester (NHS) }\end{array}$ \\
\hline & & $\begin{array}{l}\text { epoxy } \\
\text { aldehyde }\end{array}$ \\
\hline$-\mathrm{SH}$ & Cys & $\begin{array}{l}\text { maleimide } \\
\text { pyridyil disulfide } \\
\text { vinyl sulfone }\end{array}$ \\
\hline$-\mathrm{COOH}$ & Asp, Glu & amine \\
\hline$-\mathrm{OH}$ & Ser, Thr & epoxy \\
\hline
\end{tabular}

Table 2.1 Functional groups in a protein and the required functional groups in the surface for covalent binding [14].

\subsubsection{Bioaffinity Immobilization}

Bioaffinity relies on the chemical affinity of complementary molecules. The specificity of the molecules ensures an immobilization of protein that is uniform, ordered and strong with bonding that can be reversed without denaturation of the molecules, by changing conditions of the assay. There are two ways to have bioaffinity immobilization of molecules [20]. The first method consists of the immobilization of the protein/enzyme onto a surface that contains a ligand with high affinity for the protein/enzyme. In the second strategy, the protein/enzyme does not have affinity to the ligand attached to the surface, rather, is conjugated to a tag with high affinity to the ligand. Figure 2.1 shows the two types of bioaffinity immobilization of proteins. The two most common forms of bioaffinity pairs used in research are biotin-avidin and antibody-antigen pair. Biotin and avidin bioaffinity system were used in the studies presented here. 


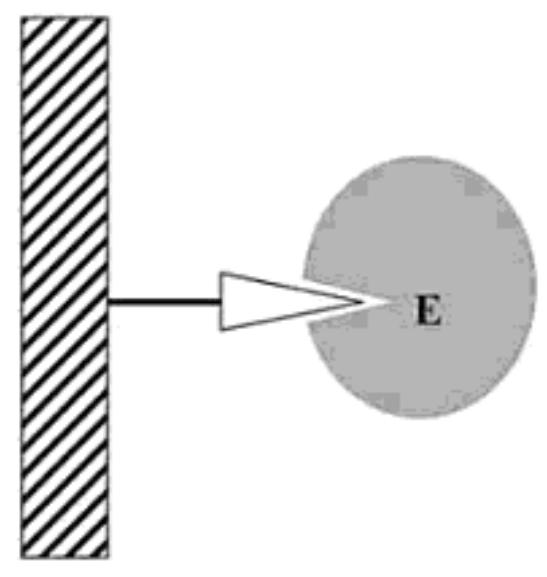

A

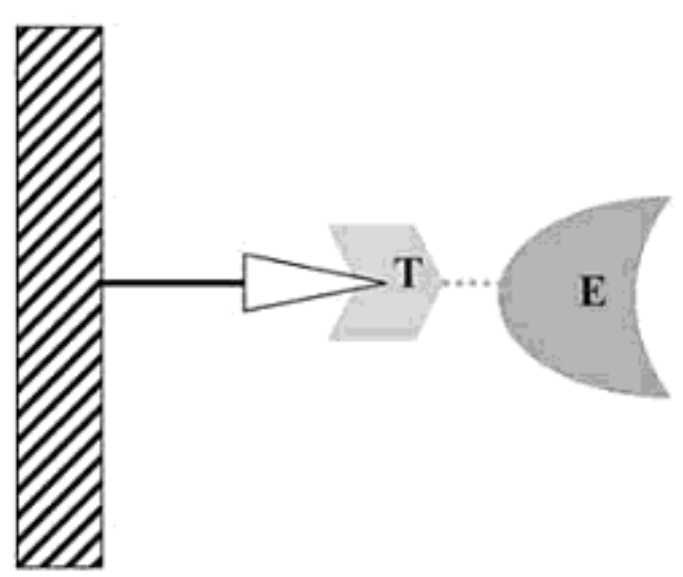

B

Figure 2.1 Types of bioaffinity immobilization A) direct; the protein is immobilized by the high affinity with the ligand anchored in the surface B) indirect; the protein does not have affinity with the ligand but it $s$ conjugated with a tag that have indeed affinity with the ligand [20].

\subsection{Methods of Protein Immobilization}

Several strategies have been studied to achieve selective immobilization onto different substrates. These methods can be classified in two distinct categories: direct and indirect [21]. There are many methods that can be classified into these two categories to selectively immobilize protein (spatial immobilization) in substrates but having a comprehensive review of these methods is beyond the scope of this thesis; therefore, this section reviews only some strategies for selective immobilization of proteins. 


\subsubsection{Direct Methods}

These methods are those referring to techniques in which proteins are directly transferred to the surface without previous chemical modification of the surface to produce a reaction with the molecule to be immobilized [21]. The transferring of the protein is done by a process known as microcontact printing $(\mu \mathrm{CP})$ a technique developed by Whitesides and collaborators in 1993 as an alternative to more complex processes such as photolithography [21]. This technique is based on a flexible replica that is used to transfer molecules directly to the surface, similar to stamping ink onto paper. Other similar techniques based on the fabrication of a flexible replica molding such as replica molding (REM), microtransfer molding ( $\mu \mathrm{TM}$ ), micromolding in capillaries (MIMIC), or solvent-assisted micromolding (SAMIM) have been successfully demonstrated as alternative ways to pattern micro and nanostructures [22].

The microcontact printing method relies on the fabrication of flexible replica usually made of poly-dimethylsiloxane (PDMS) that is placed on a rigid master (Figure 2.2). This rigid master is fabricated using microfabrication techniques like photolithography or ebeam writing or from less expensive methods such as diffraction gratings [22]. 
A
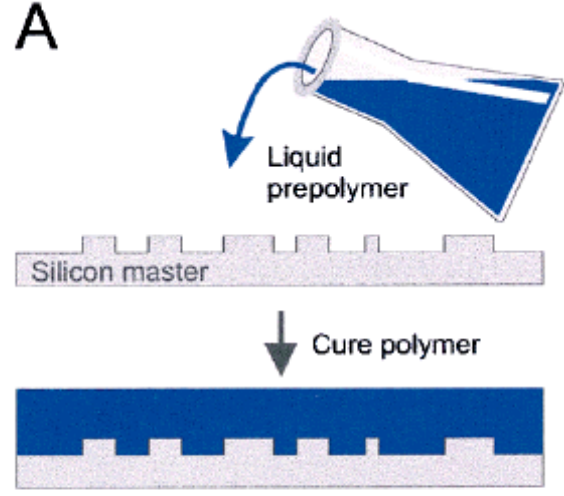

Release replica

Elastomeric stamp
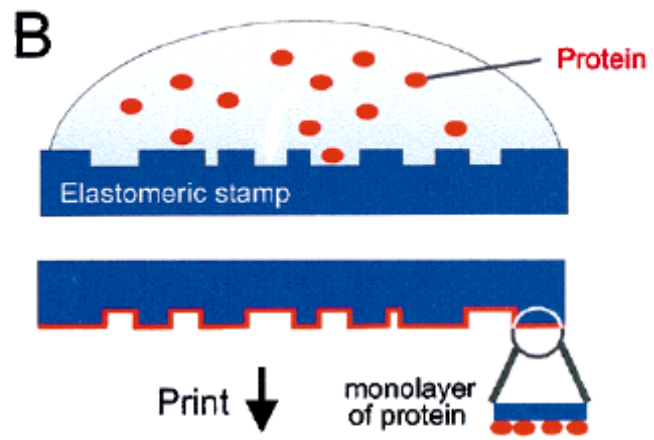

Substrate

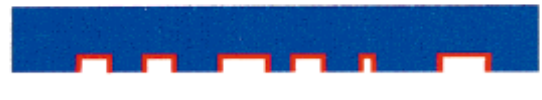

Figure 2.2 Microcontact printing of proteins. A) PDMS fabrication process B) Protein transfer into the surface [21].

This PDMS material is a polymer with unique properties, one of which is flexibility. PDMS has the lowest glass transition temperature, meaning that PDMS is composed of a flexible chain of molecules and is more difficult for the material to become glassy or more rigid [23]. Other properties that make PDMS useful are permeability, compatibility (most molecules in contact with PDMS do not react or change their properties), optical transparency, and stability to temperature fluctuations.

The PDMS material is commercially available. PDMS kits contain two elements in liquid form that must be mixed: base agent and curator. The typical mixing ratio used for PDMS stamp fabrication is (10:1), however chemical or mechanical characteristics can be modified if other ratios are used [24]. The curing process is done usually at high temperatures but the material can cure at room temperature if left for more than 48 hours $[25]$. 
The surface of PDMS is hydrophobic, meaning that the surface has low affinity to water molecules. In some applications, such as microfluidics, the process requires a change of wetting properties from hydrophobic to hydrophilic. In the transferring of molecules using microcontact printing, wettability properties play a role. Tan and colleagues studied micropatterning of proteins on different types of self assembled monolayers (SAM). They suggested that transferring of SAMs from the stamp to the surface can be related to wettability differences between the stamp and the surface. For proteins, they found that transfer of protein occurs when the stamps was more hydrophobic than the surface; however, this transferring mechanism is still to be determined [26]. They also found that the protein immobilization mechanisms in microcontact printing differ from those in physical adsorption.

The advantages of microcontact printing are numerous: it does not require complex chemical processes and the transferring of protein is quick [21]. Disadvantages are that functionality of molecules can be compromised after immobilization and there is low efficiency of the transferring process [27]. In addition, the reproducibility of the patterns in between experiments is compromised because the patterns are not homogeneous due to lack of spatial control. The low stability of the transferred protein after several washes is also an issue, which is particularly important for development of a complex biomolecular system. Finally, there are issues related to toxicity if the PDMS stamp is not well cured before use. 
Recently investigations in microcontact printing techniques have focused on the problem of molecular activity of patterned molecules. Work conducted by Trimbach and colleagues showed a comparison in protein transferring using a conventional PDMS with a hydrophilic stamp fabricated from polyether-ester (PEE). They concluded that activity of the transferred protein from PEE stamps was likely preserved because the stamp was more hydrophilic. In addition, they found that quality of the micropatterns using the PEE stamp were far superior than those using PDMS stamp, even at low protein concentrations [28]. Therefore, despite its limitations, the micropatterning contact is a valid alternative to selectively immobilized surfaces and more research is warranted to improve the performance of this method.

Other direct methods include the dip pen nanolithography (DPN) patterning or the use of lasers to directly pattern molecules onto substrates, known as matrix-assisted pulsed laser evaporation direct writing (MAPLE-DW). In DPN, an AFM tip is used as a tool to directly "write" patterns of molecules in solid substrates (Figure 2.3). The working principle utilizes an AFM tip inked with the desired molecules and placed onto the surface. Through capillary transport mechanisms, the molecules are "written" [29]. Lim and colleagues used this technique to produce arrays of proteins in modified silicon oxide surfaces. However, they did not demonstrate that the activity of the proteins was preserved, a key factor still under investigation [30]. 


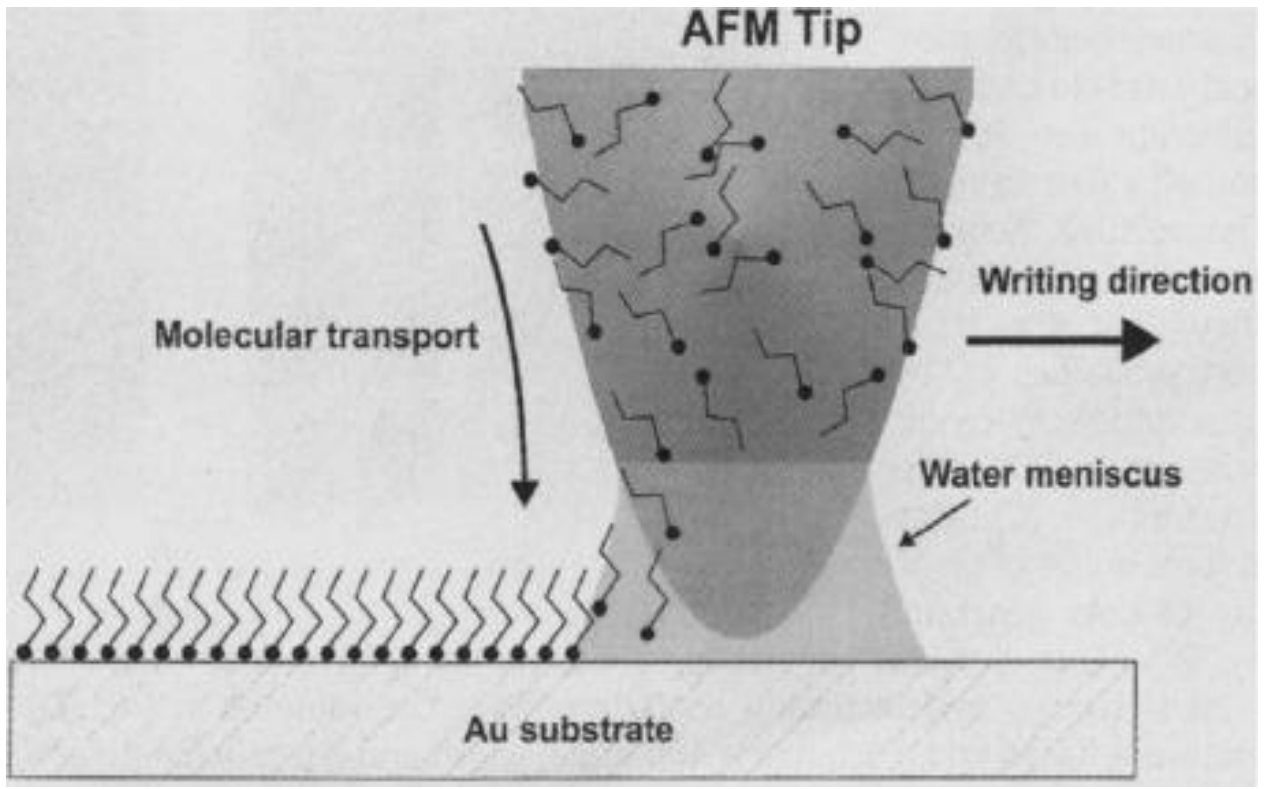

Figure 2.3 Schematic representation of the dip nanotechnology printing technique [24].

The MAPLE-DW method is based on the use of a laser that ablate a surface known as a ribbon, which is an UV transparent quartz material that is coated with the desired molecules to be patterned [31]. Protein patterning using this strategy has been previously reported [32]. Dinu and colleagues compared this novel technique to the established technique of microcontact printing, and concluded that MAPLE-DX patterning offers more homogeneous patterns and better resolution for small features [32]

\subsubsection{Indirect Methods}

Indirect methods are those techniques that rely on chemical processes that alter the functionality of the substrate or elements that are deposited on the substrate that serve as crosslinker agents between the surface and proteins to be immobilized. Proteins can be immobilized using $\mu \mathrm{CP}$ of SAMs (self assembled monolayers) followed by the 
incubation of this patterned SAM with proteins. Mrksich and collaborators reported the patterning of fibronectin protein using the above procedure. They created patterns of alkanethiolates in gold surfaces followed by two hours of incubation with the protein with the alkanethiol pattened surface, resulting in the adsorption of the proteins by the methyl groups present in alkanethiols patterns [33].

Other ways to indirectly pattern protein include the use of crosslinker agents to establish a covalent bond between the surface and the molecule, or through the use of photolithographic techniques. An example of this photolithography method was demonstrated by Lee and colleagues. They used standard photolithography to pattern photoresist in silicon dioxide in combination with polyethylene glycol (PEG) followed by a silanization process using APTES (3-aminopropyltriethoxysilane) and glutaraldehyde which was used as a crosslinker agent between the amine surface and the protein. This process created a patterned surface with PEG (protein blocker) and amine-aldehyde areas. They used protein A and fluorescent IgG to assess the selective patterning [34].

Other indirect methods include the photochemical modification of SU-8 photoresists with anthraquinone photolinkers (AQ) [35]. This method consists of the exposure of the SU-8 photoresist with the AQ agents followed by the standard photolithography process (Figure 2.4). 


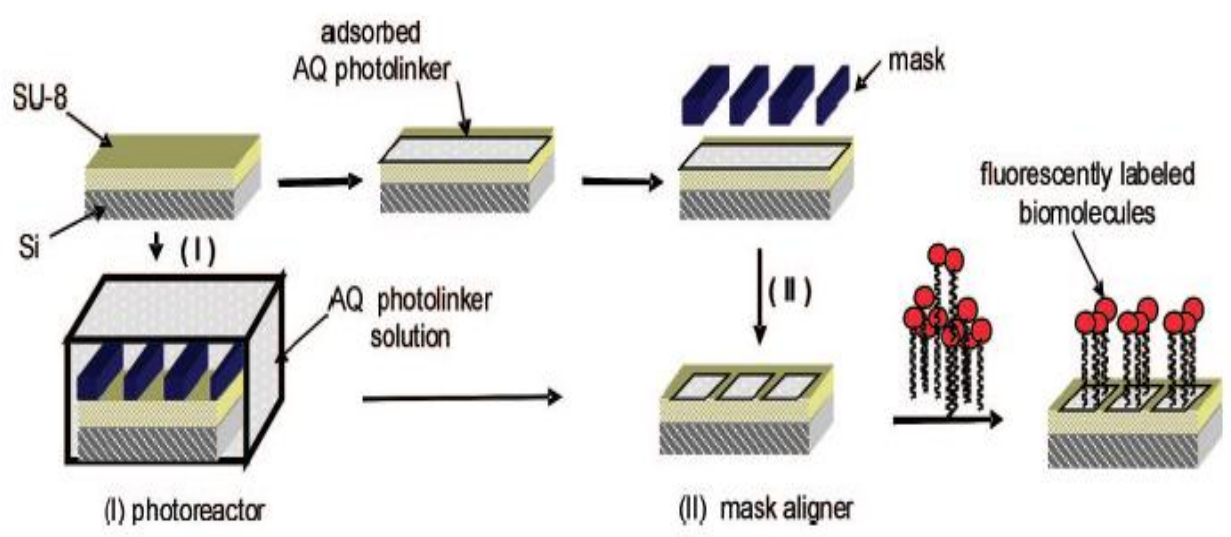

\section{Figure 2.4 Schematic process of patterning of biomolecules using chemical modification of SU-8 photoresist [35].}

Similar methods involving other types of photopolymer materials have been studied by researchers with the emphasis of creating small features in the micrometer range with the same resolution and quality as the typical photolithography process used in microfabrication of electronic devices [36].

Another attractive method is to make use of chemically modified molecules like biotin coupled with streptavidin protein. This type of immobilization consists of the photochemical modification of biotin with an extra aryl-azide group, which upon UV irradiation undergoes structural changes generating nitrenes radicals that are reactive towards C-H bonds. Hengsakul et al. (1996) investigated this type of photochemical immobilization using photobiotin to create patterns of avidin on functionalized surfaces with a mask for selective patterning of the photo-modified biotin [37]. A suitable functionalized surface is required to successfully bind this photoderivative of biotin to the surface. Silanization is one of the most common methods to acquire functional groups on 
a substrate, although the layer formation is highly dependent on the hydroxylated groups present on glass surfaces. To create these hydroxylated groups on the glass surfaces it is necessary to have a cleaning process where any organic residue can be reduced or eliminated. The way these layers are formed on clean glass slides is through the formation of polysiloxane groups that are linked to the surface silanol groups [38].

The most common chemical compound to have functionalized amino-silane surfaces is APTES. This element is used to create a suitable surface in which UVexposed photobiotin can establish a covalent bond. Other researchers have used photochemical immobilization to create patterns on surfaces other than glass or by using glass substrates functionalized with different chemical groups [39, 40, 41]. Amino-silane type of photochemical modification was studied in the studies presented in this thesis (described in Figure 2.5).

In summary, indirect methods that involve photolithographic processes or photochemical immobilization, produce patterns which are homogeneous, very reproducible, stable (due to strong binding) and have high resolution in the micrometer range. Together, these factors make indirect photolithographic processes well suited for selective patterning of proteins onto glass surfaces. 


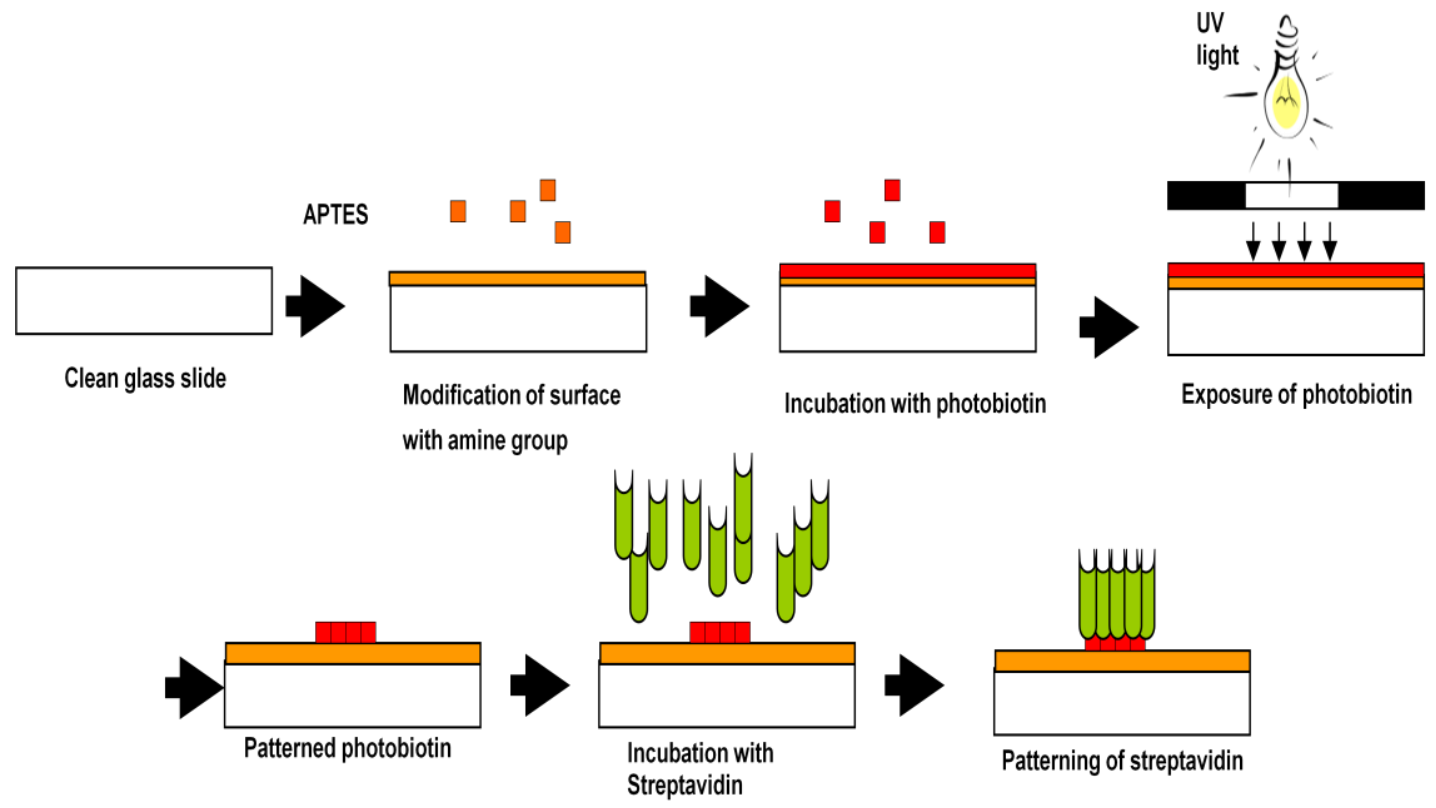

Figure 2.5 Schematic process of patterning of streptavidin using photochemical activation of photobiotin.

\subsection{Actin Motor Protein Structure and Functionality}

Actin is a $42 \mathrm{kDa}$ protein found in two forms in eukaryotic cells: globular or monomer G-actin and filamentous actin [42]. Actin has an important role in cell shape, cell motility as well as for muscle contraction [43]. The globular form of actin can be polymerized to form filamentous actin in the presence of physiological concentrations of salts and through hydrolysis of adenosine triphosphate (ATP). This polymerization process is reversible, therefore filamentous actin can be disassembled into globular actin, forming the basis for the dynamic nature of the eukaryotic cytoskeleton [44]. Actin is composed of 375 amino acid residues $[42,45,46]$. The amino acid chain is folded in two domains and further divided into four sub-domains. These large domains are organized in a molecule with a deep cleft in which a nucleotide binds [46] (Figure 2.6). 


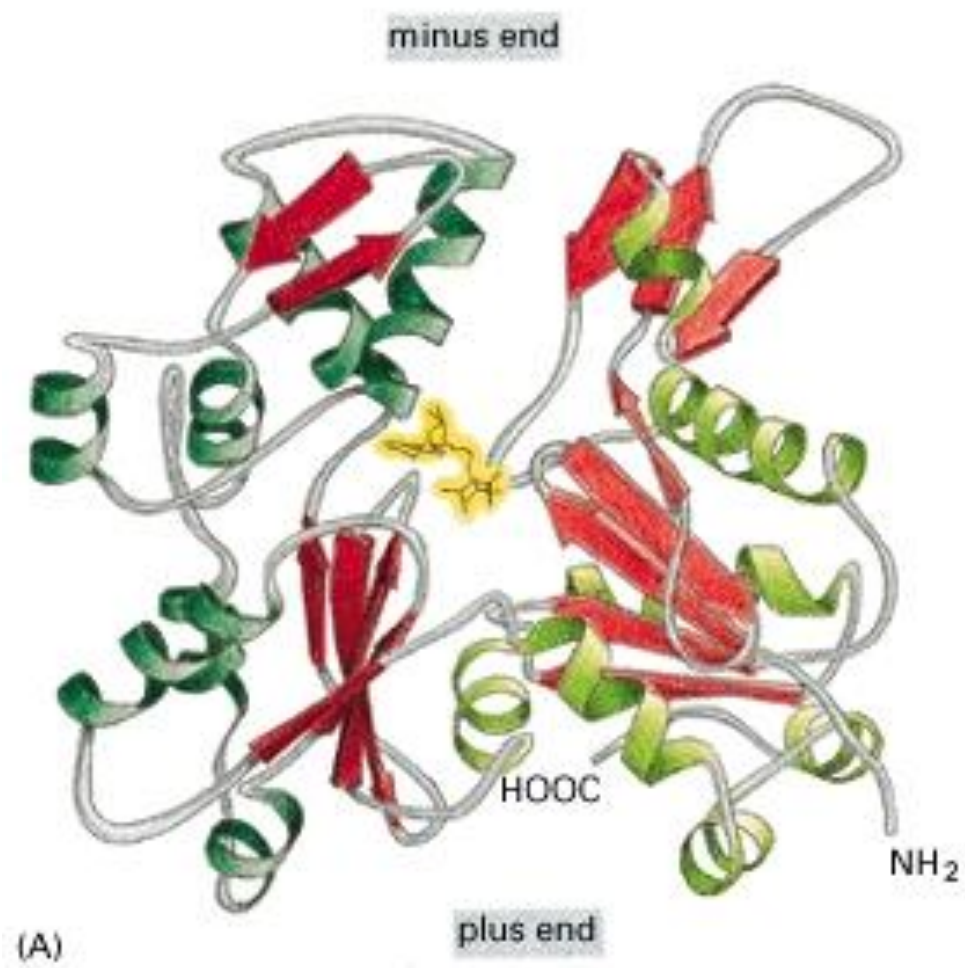

Figure 2.6 Representation of the molecular structure of globular actin, showing the plus and minus ends as well as the bound nucleotide (yellow molecule) [47].

This adenosine nucleotide interacts with cations such as $\mathrm{Mg}^{2+}$ or $\mathrm{Ca}^{2+}$. There are differences in protein kinetics, depending on which type of cation interacts with the nucleotide [42]. If $\mathrm{Mg}^{2+}$ is present, the disassociation of ATP is faster. The subunits of actin or G-actin assemble with the cleft oriented in the same manner. Upon hydrolysis, the bound nucleotide disassociates into adenosine phosphate (ADP) and inorganic phosphate while undergoing a structural change with the ADP molecule closed and embedded (Figure 2.7). 
Polymerized actin consists of two actin strands wrapped in a helix structure with a diameter of aproximetely $7 \mathrm{~nm}$. Both ends of the polymerized actin can grow but the rate of assembling is different because the association constant values are
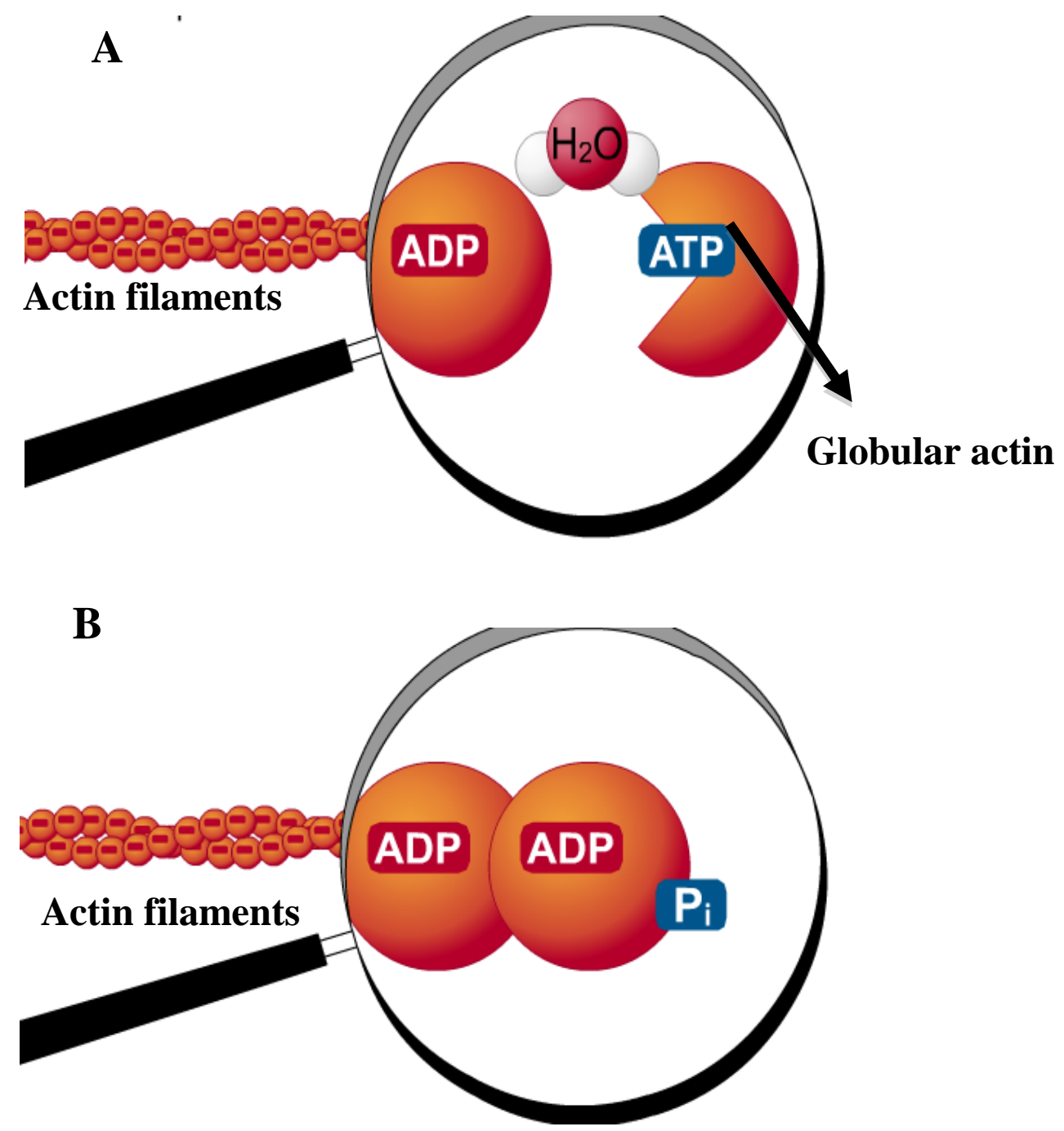

Figure 2.7 Representation of the polymerization of actin filaments A) Hydrolysis of ATP B) conversion of ATP to ADP and inorganic phosphate [48].

different. The plus end (barbed end) has a higher rate of growth than the minus end (pointed end). Actin flaments have a defined polarity which has been demonstrated by observation of arrow shapes structures upon attachment of myosin S1 subfragments [42]. 
The dynamics of actin polymerization and depolymerization plays a key role in the shape of cells and is involved in cells signaling as well. The dynamics of the filamentous actin can be altered through binding of regulatory proteins such as gelsolin.

\subsection{Biotin-Streptavidin Structures and Functionality}

The biotin and streptavidin system is a well known type of ligand-antigen system. The high affinity of the protein streptavidin to the small biotin vitamin $\left(K_{\mathrm{a}}=10^{15} \mathrm{M}^{-1}\right)$ [49], represents the strongest form of non-covalent bond, which is stable even in harsh conditions such as $\mathrm{pH}$ changes or other denaturing conditions. This stability makes this bioaffinity system attractive for the implementation of detection assays or targeting systems.

Biotin is a $244 \mathrm{Da}$, water soluble vitamin present in almost every living cell [49]. This vitamin acts as co-factor in the conversion process of food to energy and also plays a significant role in the metabolism of fatty acids and control of blood sugar [50]. The structure of biotin consists of a tetrahydroimindizalone ring embedded into a tetrahydrothiophene ring. Another structural characteristic is the presence of a valeric acid instead of a hydrogen atom, bound onto one of the carbon atoms in the terahydrothiophene ring. One of the significant advantages of biotin is its size, which contributes to its molecular stability.

Streptavidin is a tetrameric protein derived from the Streptomyces avidinii actinobacterium. Each monomer has a binding site that has high affinity with biotin. Avidin is a protein similar to streptavidin and can also form tight bonds with biotin in its four binding sites. A disadvantage to the use of avidin in ligand-antigen systems, is the 
prevalence of non-specific binding, which is mainly caused by sugars and high pI (isoelectric point) [12]. There are also considerable structural differences between avidin and streptavidin [51], the most notable of which is the arrangement of amino acids giving avidin a higher $\mathrm{pI}$ value and altering the isolectric point [51].

The biotin-streptavidin system has been extensively studied and modeled to understand the fundamentals behind the strong biotin and streptavidin interaction. Various studies have shown that streptavidin is arranged in a D2 type symmetry. Each subunit in the protein folds into a specific antibarrel $\beta$ shape. Biotin then binds into open pockets at the end of this antibarrel $\beta$ shape structure [52]. Once the biotin binds, it is locked into place by a surface loop (formed by residues 45-52) that fold on top of the binding site (Figure 2.8). The tight binding is attributed to various factors such as van der Waals forces and hydrogen bonding [53].

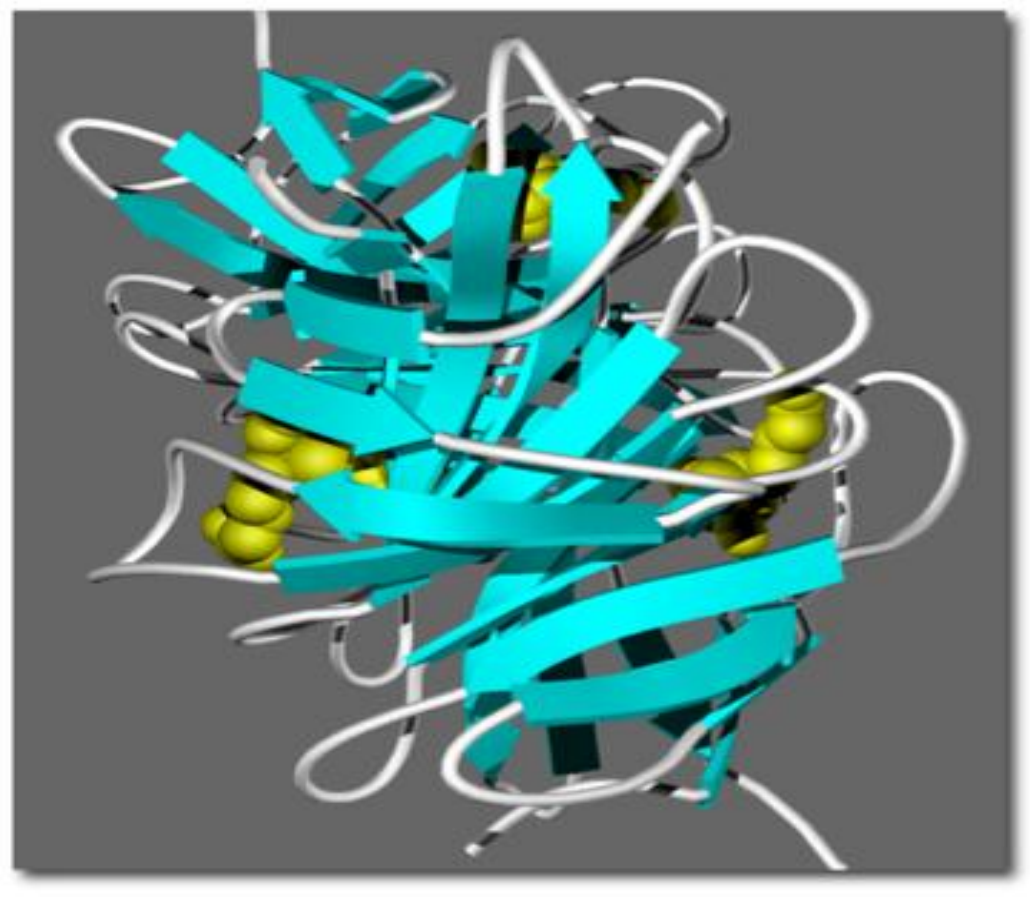

Figure 2.8 Depiction of the molecular structure of streptavidin, the yellow elements represents biotin bound at the open sites of the antibarrel $\beta$ shape structure [54]. 
The study of the biotin and streptavidin system is not limited to their structure and binding characteristics, however. Another parameters under investigation are the exposure of this system to extreme assay conditions. Holmberg and colleagues reported that breaking the biotin-streptavidin non-covalent bond without damaging either molecule could be achieved by exposing the system to temperatures higher than $70 \mathrm{C}$ in non-ionic buffer [55]. These results offer the possibility of reusing these molecules multiple times by controlling the environmental conditions of the assay, making this bioaffinity method desirable for detection of multiple targets using the same sample. Computer modeling of binding dynamics or the force need to break the bonds between biotin and streptavidin have also been explored $[56,57,58]$.

\subsection{Gelsolin Regulatory Capping Protein}

Gelsolin is a calcium ion-dependent protein that acts to regulate control of actin filament length. Gelsolin binds actin and disrupts the weak non-covalent bond between actin subunits, severing the filament [59]. Following breakage of the filament, gelsolin remains attached to the end of the actin filament (the plus end), preventing the filament from association with more actin subunits, thereby decreasing the rate of polymerization $[60,61]$. Regulation of gelsolin activity can be achieved through manipulation of calcium ion availability and $\mathrm{pH}$ of the system. Calcium concentration in the micromolar range and $\mathrm{pH}$ less than 6.5 disrupt activity of gelsolin [62].

The structure of gelsolin consists of six domains, subdivided into two halves (S1$\mathrm{S} 3$ and S4-S6). These two halves are known as $\mathrm{C}-$ and $\mathrm{N}$ - which are connected through a linkage molecule [59]. The C- half binds to actin only when the levels of calcium are 
sufficiently high $\left(10^{-6} \mathrm{M}\right)$. The second half of the molecule (N- half) can bind and sever the actin filament even when calcium is not present. However, this process does not occur with any consistency due to inhibition of this activity by the C- side. Therefore, in order for gelsolin to sever actin filaments, levels of calcium must be sufficiently high to enable both halves to work in conjuction. Severing and capping of actin filaments occurs in a step-wise, predictable process. First, the capping protein undergoes an unlatching process in which the protein unfolds from its default quartenary structure in the presence of $\mathrm{Ca}^{2+}$. Domains S2 and S3 from the N- side bind actin with S1 positioned between the edges of two globular actin molecules. This positioning is followed by structural changes that allow the linker between $\mathrm{S} 1$ and $\mathrm{S} 2$ to elongate such that $\mathrm{S} 1$ occupies the space between actin subunits. S4 and S6 then bind to actin at the other strand of the filament. Finally, after enough bonds are broken, the filament is severed and domains S1 and S4 position themselves onto the double strand, capping the actin molecule [59] (Figure 2.9).

This capping of actin filaments is reversible and is not $\mathrm{Ca}^{2+}$-dependent. To disassociate gelsolin caps from the filament phosphoinositides are required. Phosphatidylinositol 4,5-bisphosphate $\left(\mathrm{PIP}_{2}\right)$ is especially effective. $\mathrm{PIP}_{2}$ binds the N-half of gelsolin producing structural changes between domains S1 and S2 thereby disrupting their bond to actin. The attachment of PIP $_{2}$ to gelsolin is greatly increased by the presence of $\mathrm{Ca}^{2+}$ and low $\mathrm{pH}$ values [59]. 


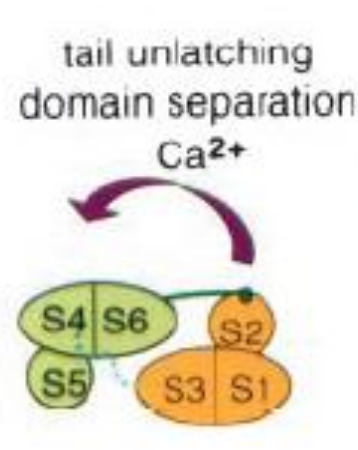

Activation

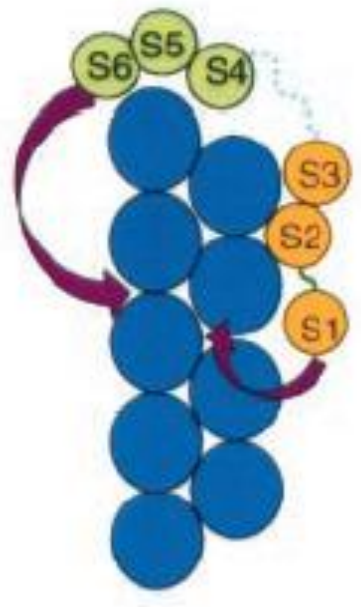

Severing

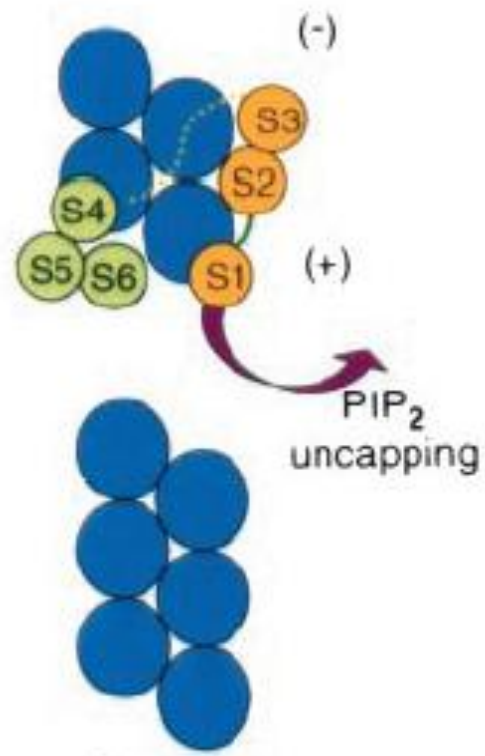

Capping

Figure 2.9 Process of activation, severing, capping and uncapping of gelsolin. The process begins with the unfolding of the protein in the presence of $\mathrm{Ca}^{2+}$, followed by the binding, severing and capping of actin. The process can be reversed by exposing the capping protein to $\mathrm{PIP}_{2}[59]$. 


\section{CHAPTER 3 METHODS AND PROTOCOLS}

In this chapter, a description of methods and protocols used in this thesis are explained in detail. The first section of this chapter describes the process of designing a photofilm mask, which is a tool used for selection of areas to be patterned in both of the protein immobilization methods used in the studies presented in this thesis. The results obtained in this section are discussed in Chapter 5.

\subsection{Photofilm Mask Design}

The photofilm mask was used for specific selection of areas to be patterned on the glass substrate for immobilization of photobiotin, and for the fabrication of a silicon master in the microcontact printing method. The photofilm mask design was created with the CAD program Corel Draw x4 (Corel). Photofilm mask features were created to fit both formats $(18 \times 64 \times 25 \mathrm{~mm}$ for glass slides and $75 \mathrm{~mm}$ diameter for the fabrication of silicon masters). Masks contained lines, curves and segmented lines, with different thicknesses ranging from $10 \mathrm{um}$ to $100 \mathrm{um}$, with length of $3 \mathrm{~mm}$, and separation between features ranging from 10um to 500um (Figure 3.1). For features smaller than 10um, a chrome mask, phase shift mask, or different photolithography process, such as E-beam lithography, would be required due to diffraction ans subsequent poor resolution. The design files were sent to a business outside West Virginia University (Photoplotstore; Denver, CO) that specializes in printing precise laser plots. 


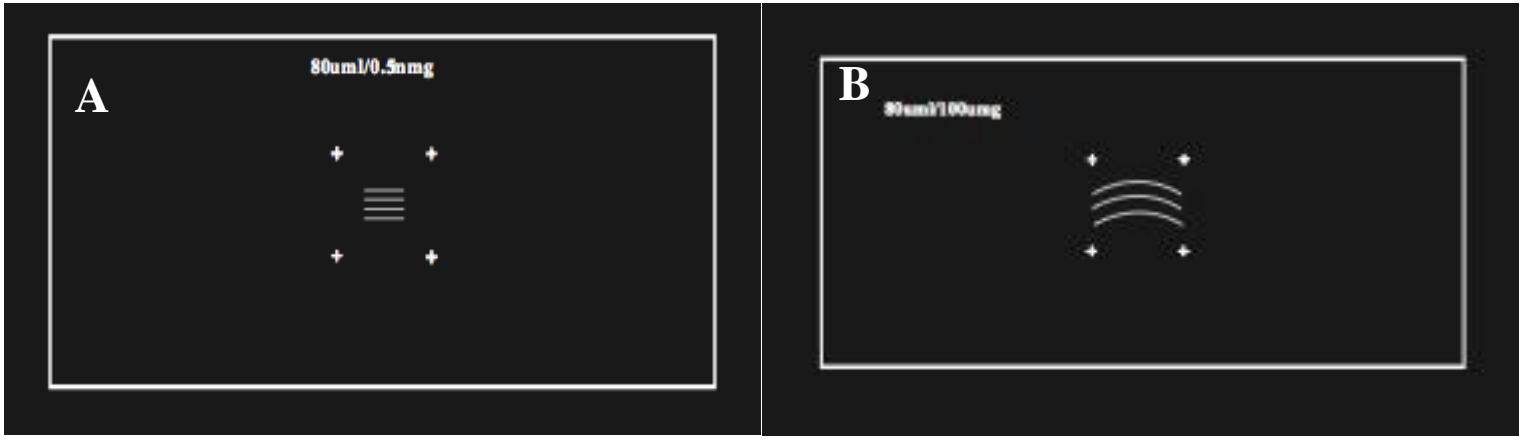

Figure 3.1 Photofim masks A) lines features are $80 \mu \mathrm{m}$ and separation between features is $0.5 \mathrm{~mm} \mathrm{B)} 80 \mu \mathrm{m}$ curves features.

\subsection{Photochemical Immobilization Process}

\subsubsection{Cleaning of Glass Substrates}

One millimeter thick, $1 \times 3$ inch and $2 \times 3$ inch glass microscope coverslips (Fischer Scientific) were cleaned using the following protocol. Slides were immersed in a Petri dish containing acetone and sonicated in ultrasound bath for 5 minutes. The same process of ultrasound sonication was repeated using methanol for an additional 5 minutes followed by drying with nitrogen gas $\left(\mathrm{N}_{2}\right)$. This process serves to reduce the organic residue on the slide. Next, the slides were placed in a holder and immersed in a beaker containing $300 \mathrm{ml}$ of Summa Clean (trimethyl (2-hydroxyethyl) ammonium hydroxide, \#8006-08 Mallinckrodt Baker, Inc), solution heated to 55-60 C, for 30 minutes to completely eliminate any organic residue. Afterwards the slides were rinsed with water for 5 minutes and dried with nitrogen gas. The cleanliness of the slide was verified by observing water tension on the surface as determined by dropping water onto the slide surface. A clean slide has a highly hydrophilic surface (the surface of the slide has hydroxyl groups) and water dropped onto the slide disperses rather than forming beads. 

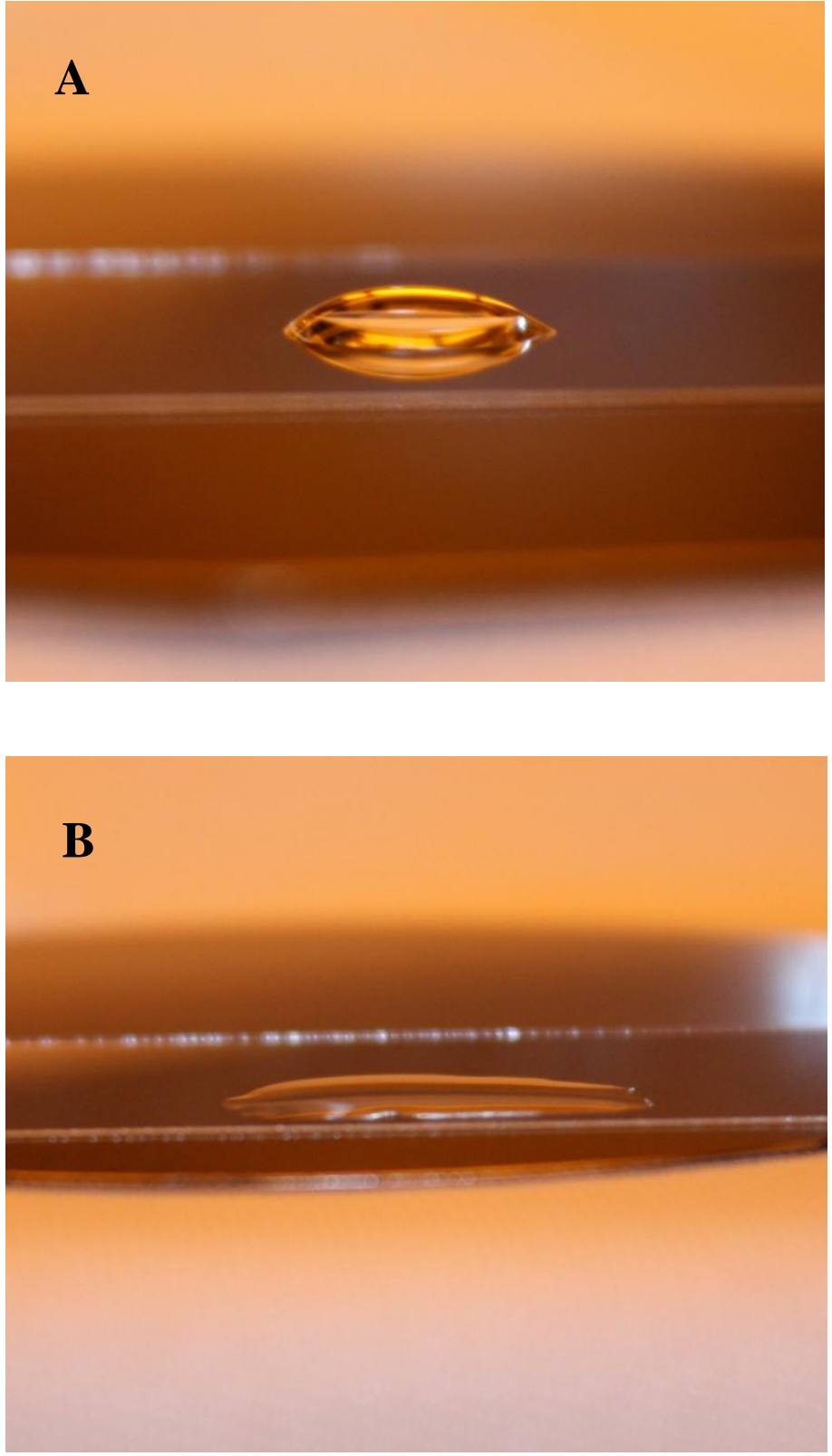

Figure 3.2 Glass substrates A) untreated sample B) treated clean slides 


\subsubsection{Silanization of Glass Substrates}

The silanization of glass slides was completed after the cleaning process. A solution of 3\% v/v of APTES (aminopropyltriethoxysilane) (Sigma Aldrich) in anhydrous ethanol was prepared and sonicated in ultrasound bath for 10 minutes to ensure proper mixing. The clean slides were then immersed in 3\% v/v APTES in a Petri dish and incubated for 30 minutes. The modified slides were rinsed three times with ethanol, deionized water and dried with nitrogen gas. The slides were placed in an oven for 20-30 minutes to anneal the APTES and form the silanol groups. After the baking process, the amine functionalized glass slides were stored in deionized water for later use.

\subsubsection{Photobiotin Deposition}

The process of depositing photobiotin, 3-[3-(4-azido-2-nitroanilino)-Nmethylpropylamino]propylamide acetate salt (Sigma Aldrich) began by restoring the photobiotin from lyophilized form to liquid form by adding $1 \mathrm{ml}$ of milliQ pure ( $3 \mathrm{X}$ deionized water, conductivity $=18 \mathrm{M} \Omega . \mathrm{cm}$ ) to $1 \mathrm{mg}$ of the compound for a concentration of $1 \mathrm{mg} / \mathrm{ml}$. Stocks of ten times diluted solutions $(0.1 \mathrm{mg} / \mathrm{ml})$ were prepared for the experiments and stored at $-20^{\circ} \mathrm{C}$ for later use. The deposition of the photobiotin was achieved by pipetting $30 \mu \mathrm{l}$ of the photobiotin solution $(0.1 \mathrm{mg} / \mathrm{ml})$ onto the amine group functionalized glass slide. The samples were then placed on a dessicator under vacuum for one hour to evaporate the water content and to get a thin film on the glass substrate. An alternative procedure was to spin the photobiotin on the glass slide at $400 \mathrm{rpm}$ for 90 seconds followed by a baking process in which slides are placed on a baking plate for 30 minutes at $40^{\circ} \mathrm{C}$. 
After the photobiotin deposition, samples were exposed to UV light (OAI 0130040-03 flood exposure) with a wavelength of 365nm (near UV light) and intensity of approximetely $9 \mathrm{~mW} / \mathrm{cm}^{2}$. UV exposure involved placing a photofilm mask to create the desired patterns on the photobiotin film and exposing slides to the UV light for 5 minutes. Next, the samples were placed in de-ionized water and sonicated in ultrasound bath for 10 minutes to remove unbound photobiotin. A small cover slip $18 \mathrm{~mm} \times 18 \mathrm{~mm}$ (Dow Corning) was placed on top of each sample separated by small pieces of 3M double-sided tape with a thickness of $0.156 \mathrm{~mm}$ to act as a separator and to create a flow cell to inject protein solutions (Figure 3.3).

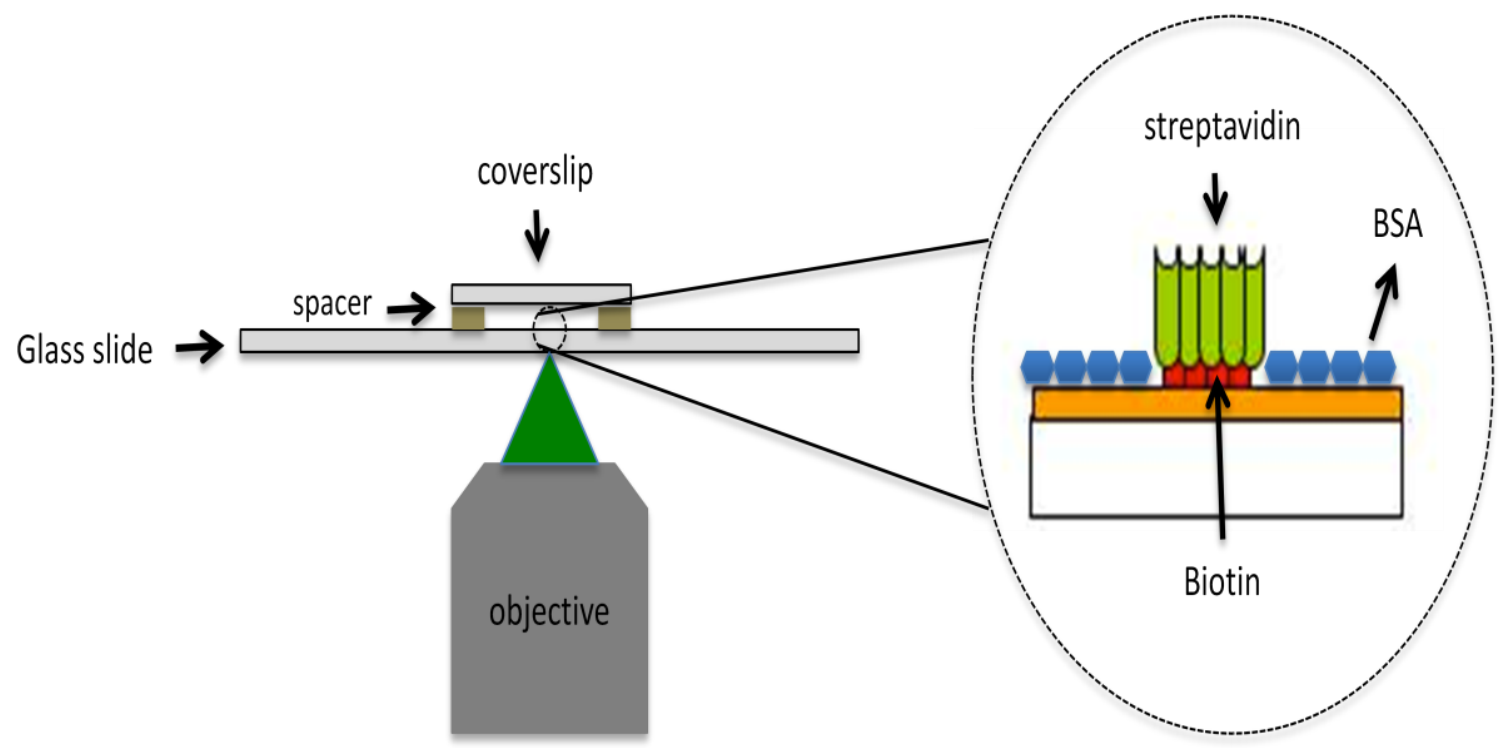

Figure 3.3 Schematic of the flow cell used during experiments. On the right is a magnified view of the flow cell showing the biological elements present.

After the flow cell was created, $40 \mu \mathrm{l}$ of $1 \% \mathrm{v} / \mathrm{v}$ of Tween20 (Polysorbate 20, Sigma Aldrich) in de-ionized water was injected and incubated for one hour. This solution is used to prevent non-specific binding of streptavidin protein. An alternative method of preventing non-specific binding is to incubate the slide in $3 \% \mathrm{w} / \mathrm{v}$ bovine serum albumin 
(BSA) for one hour. A solution containing FITC or TRITC streptavidin (Pierce) was then prepared by diluting streptavidin (in lyophilized form) into PBS buffer (0.1 M sodium phosphate, 0.15 M sodium chloride, $\mathrm{pH} 7.2$; Thermo Fischer) to a final concentration of $0.08 \mathrm{mg} / \mathrm{ml}$. This streptavidin solution was then injected and incubated with photobiotin patterned samples for one hour. Finally, the samples were washed thoroughly with PBS buffer and placed under the fluorescent inverted microscope (Nikon EclipseTE-200) for observation and data acquisition.

\subsection{Microcontact Immobilization Process}

The second process that was used in the studies outlines in this thesis was

microcontact printing. This method is based on the transferring of molecules using a stamp, which is made of a flexible material and is a negative copy of the features created in a SU-8 silicon wafer master.

\subsubsection{Cleaning of Glass Substrates}

The same protocol for cleaning surfaces that was used for the photochemical immobilization process was used here as well since the cleaning of the slides was a common process for all the protein immobilization methods.

\subsubsection{Fabrication of SU-8 Master}

The fabrication of a SU-8 master was needed to create features to be transferred onto the polydimethylsiloxane (PDMS) stamp that was used for the microcontact 
transferring of proteins onto non-modified glass surfaces. The fabrication of the SU-8 master was done following a standard photolithography process (Figure 3.4). The process began by cleaning a $75 \mathrm{~mm}(<111>$ orientation $)$ silicon wafer using a similar cleaning process as the one used for glass slides described in the previous section. The clean silicon wafer is then placed in an oven for 10 minutes at $110^{\circ} \mathrm{C}$ to evaporate any remaining solvent left from the cleaning process.

The next step was to coat the silicon wafer with a layer of SU-8 photoresist. The negative process photoresist SU-8 was the photosensitive material chosen since, after development it hardens, offering a resistant material for the repetitive process of fabrication of PDMS stamps. The coating of the photosensitive material was accomplished by spinning the photoresist in a centrifuge (Lauren 4000 series programmable centrifuge) at a speed of $2000 \mathrm{rpm}$ for one minute creating a thin layer of approximately $34 \mu \mathrm{m}$. The wafer was then pre-baked by placing the wafer on a hot plate for 8 minutes at $50^{\circ} \mathrm{C}$. This pre-baking step is required to eliminate part of the solvent on the photoresist and to increase the sensitivity of the material to UV light. The wafer is then placed for a second baking process for 15 minutes at $90^{\circ} \mathrm{C}$. Following the second baking, the coated silicon wafer was exposed to UV light with a photofilm mask with the desired pattern to be transferred onto the silicon SU-8 master. After the light exposure process, the sample was developed by placing the silicon wafer in a Petri dish containing SU-8 developer for 1 minute or until all the photoresist was washed away by gently shaking the Petri dish. Finally, the developed silicon wafer master was rinsed thoroughly with acetone to remove any remaining developer left from the sample and stored for later use. 


\subsubsection{Fabrication of PDMS Stamp}

Before fabrication of the PDMS stamp, the silicon SU-8 master is treated with trichlorosilane (1,1,2,2-terahydrooctyl-1-trichlorosilane, Sigma Aldrich), to prevent irreversible adhesion of the PDMS polymer onto the silicon wafer SU-8 master. This procedure was accomplished by adding one drop of trichlorosilane in a small beaker and placing it along with the silicon master in a dessicator under vacuum overnight.

The first step in PDMS stamp fabrication was to mix the base and curator (Syglard, Dow Corning) in a 10:1 ratio. The mixture was placed in a crucible and allowed to stir for 5 minutes. After mixing, the PDMS material was placed in a dessicator under vacuum to eliminate air bubbles, in order to prevent potential problems with the material at the time of transferring features from the master to the PDMS material.

The PDMS material was then slowly poured on top of the silicon master to prevent air bubbles from forming which could affect the quality of the stamp. The next step was to place a glass cover to spread the material throughout the silicon master features and to ensure a PDMS stamp with uniform thickness. The thickness of the PDMS was set using separators with the same thickness as the cover glass used to spread the material, so the PDMS stamp would have the same thickness as the glass cover. Finally, the PDMS material was baked for 20 minutes at $120^{\circ} \mathrm{C}$ to cure the material and then carefully peeled off the master and then rinsed with acetone. A close inspection of the stamp ensures that the material is cured adequately (Figure 3.5). An uncured PDMS stamp is toxic and will cause problems with the biomolecules to be patterned. 


\subsubsection{Transferring of Protein from PDMS Stamp to Glass Substrate}

The PDMS stamp was cleaned by sonication in de-ionized water in ultrasound bath for 10 minutes. The stamp was then cleaned with oxygen plasma to increase the spreadibility of the solution on the stamp (Table 3.1).

\begin{tabular}{|c|c|}
\hline Pressure (mTorr) & 300 \\
\hline Power (Watts) & 50 \\
\hline BP/RP & 80 \\
\hline Time (Seconds) & 10 \\
\hline ENDPT & 0 \\
\hline DC (Volts) & 0 \\
\hline Gas Source (Standard cubic centimeter SCCM) & 20 \\
\hline
\end{tabular}

Table 3.1 Parameters used for oxygen plasma treatment of PDMS stamps.

A solution of FITC or TRITC streptavidin was added to clean, treated PDMS stamp and allowed to incubate for one hour. Afterwards the stamp was dried with nitrogen gas or washed with deionized water to remove any excess protein on the stamp. The next step was to place the protein-coated stamp in contact with non-modified clean glass slides and manually apply pressure to ensure complete contact between the two surfaces. The transferring time was set at one minute for all experiments. Finally, the sample was placed under the fluorescent microscope for observation and data acquisition. An alternative to manual application of the stamp was to use a substrate aligner that was 
available in the laboratory along with a microscope and adjust the pressure with a micrometer in the substrate aligner.
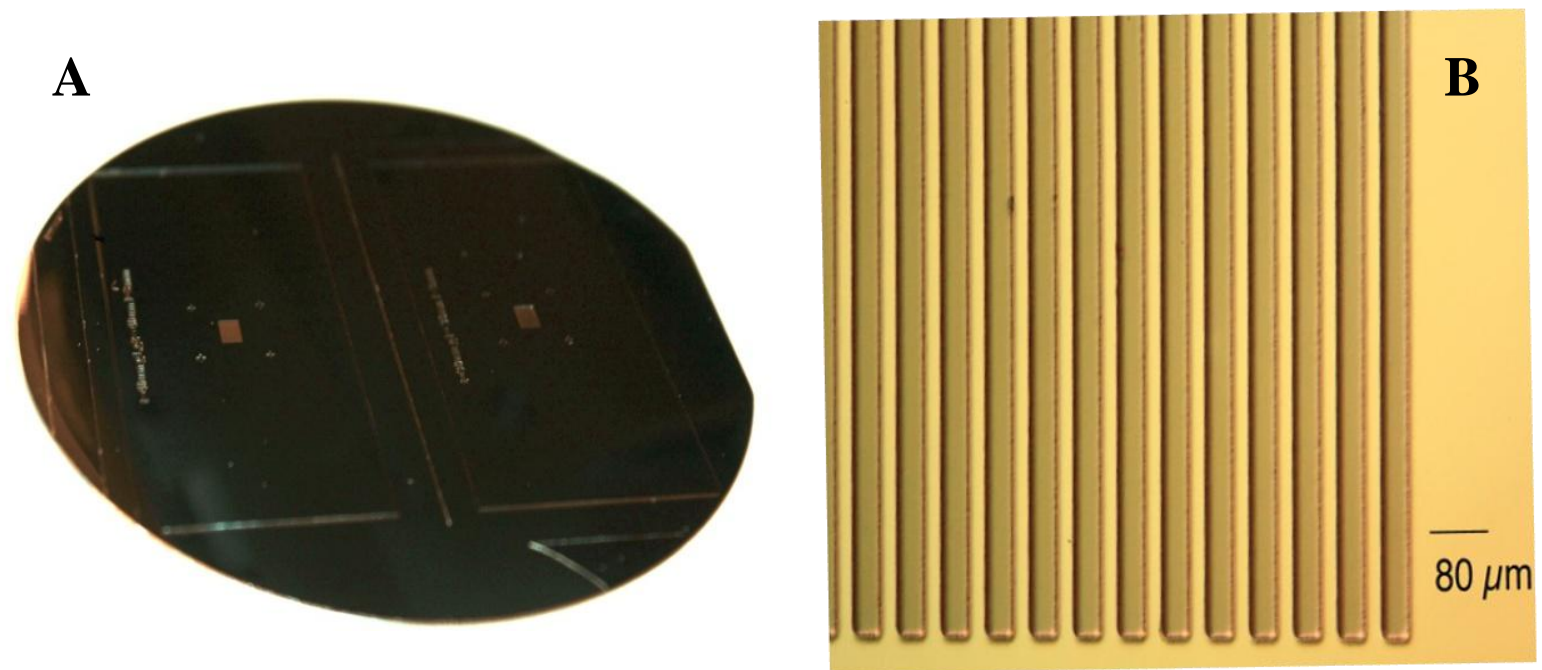

3.4 Microcontact printing master A) SU-8 silicon wafer master B) Microscope image of the SU-8 master the lines features are $40 \mu \mathrm{m}$ thick and the length is $3 \mathrm{~mm}$.

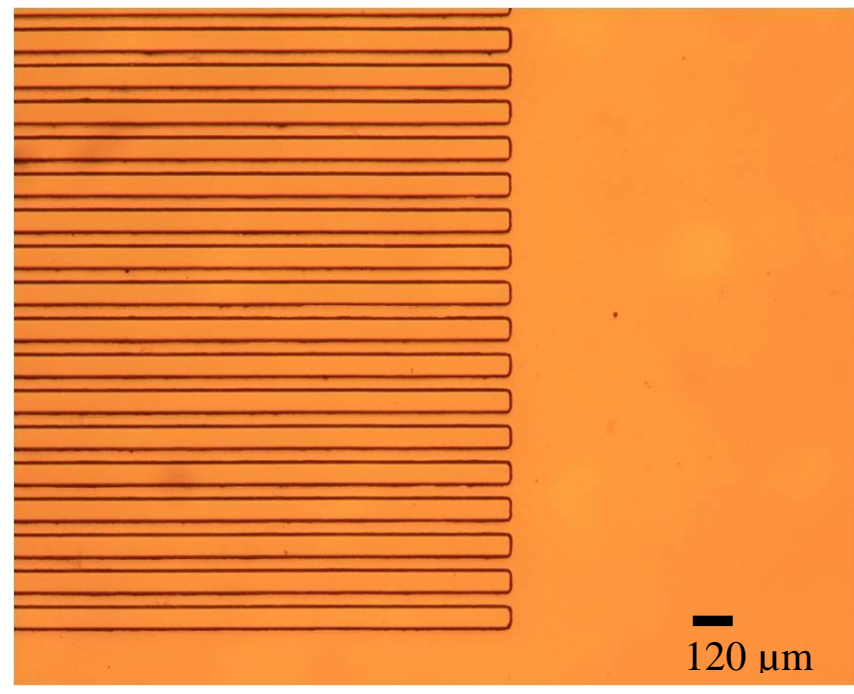

Figure 3.5 Microscopic image of fabricated PDMS stamp. Line features are $60 \mu \mathrm{m}$ thick. 


\subsection{Laying Actin Filaments on Protein Patterned Surfaces}

In this section the procedures to attach actin filaments onto protein patterned surfaces are described.

\subsubsection{Actin Filament Length vs. Concentration of Gelsolin}

This experiment was designed to find the proper length of actin filaments to be placed on the protein patterned glass surface. Glass slides were cleaned using the same cleaning protocol described previously. Clean slides were placed in $1 \% \mathrm{v} / \mathrm{v}$ of PDDA (poly-diallyldimethylammonium chloride, Sigma Aldrich) dissolved in 3X de-ionized water for 1 hour. Following incubation in PDDA, the slides were dried to eliminate the excess of PDDA on the slide. PDDA was used to create a sticky surface to randomly bind actin filaments on the substrate.

Actin from rabbit muscle acetone powder (pel-freez Arkansas, LLC) was purified by Mr. Yongkuk Lee (protocol described in Appendix A). A solution of f-actin $(0.25 \mathrm{mg} / \mathrm{mL})$ in M-buffer $\left(25 \mathrm{mM} \mathrm{KCl}, 2 \mathrm{mM} \mathrm{MgCl}_{2}, 0.2 \mathrm{mM} \mathrm{CaCl} 2\right.$ and $25 \mathrm{mM}$ imidazole, $\mathrm{pH}$ 7.0) was supplied. Actin solution $(1 \mu \mathrm{l})$ was incubated with $1 \mu \mathrm{l}$ biotinylated gelsolin capping protein $(0.125 \mathrm{mg} / \mathrm{ml}$, determined by BCA protein assay) in gelsolin storage buffer $(75 \mathrm{mM} \mathrm{NaCl}, 20 \mathrm{mM}$ Tris, $0.2 \mathrm{mM}$ EGTA and $10 \mathrm{mM}$ imidazole, $\mathrm{pH} 7.4)$ and further diluted in $95 \mu \mathrm{l}$ of motility assay buffer $(100 \mathrm{mM} \mathrm{KCl}$, $2 \mathrm{mM}$ of $\mathrm{MgCl} 2,1 \mathrm{mM}$ EGTA and $25 \mathrm{mM}$ of Imidazole) with $1 \mu 1$ of $0.0356 \mathrm{mg} / \mathrm{ml}$ of catalase, $1 \mu 1$ of $4.45 \mathrm{mg} / \mathrm{ml}$ glucose, and $9.90 \mathrm{mM}$ of dithiothreitol yielding to a final concentration of $2.47 \mu \mathrm{g} / \mathrm{ml}$ for f-actin and $1.23 \mu \mathrm{g} / \mathrm{ml}$ for biotinylated gelsolin and kept for at least an hour on ice. A flow cell was created by placing a glass coverslip with a thickness of $0.16 \mathrm{~mm}$ on top of the PDDA coated samples. Double-sided tape was used 
as separators to create the space to inject solutions in the flow cell. The actin-gelsolinbiotin solution was then injected onto the PDDA coated samples and allowed to incubate at room temperature for 20 minutes. Finally, the samples were placed under the microscope for observation and data recording.

The same experimental procedures were repeated with varying concentations of gelsolin (diluted 1:10 and 1:100). This study was conducted to determine the relationship between gelsolin concentration and f-actin length.

\subsubsection{Binding Actin Filaments on Streptavidin Patterned Surfaces.}

Glass slides were cleaned, functionalized with APTES, and photobiotin patterned by following the procedures outlined in sections 3.2.1 to 3.2.3d. Solutions of a complex containing biotinylated gelsolin at concentration of $0.125 \mathrm{mg} / \mathrm{ml}(10 \mathrm{X})$ and $0.0125 \mathrm{mg} / \mathrm{ml}$ (1X) were diluted in motility assay buffer (incubated with actin filaments at a concentration of $2.47 \mu \mathrm{g} / \mathrm{ml}$ for at least one hour in ice). This solution was then injected into the protein patterned sample and incubated for 30 minutes.

Following incubation, samples were washed three times (5 minutes each) in PBS buffer. Finally, samples were placed on fluorescent inverted microscope for image and video acquisition. 


\section{CHAPTER 4 EXPERIMENTS SEQUENTIAL DIAGRAMS}

In this chapter sequential diagrams of the processes involved in the selective patterning of actin filaments are presented. The chapter begins with the functionalization of glass with amino-silane group and continues with the photoimmobilization of photobiotin. This is followed by the incubation of fluorescent tagged streptavidin and finally the immobilization of actin on streptavidin coated areas.

\subsection{Functionalization of Glass Slides with Amino-Silane Groups}

Glass slides were functionalized following the procedure outlined in Chapter 3. The main objective was to create a thin monolayer of amino-silane groups on the surface to allow an establishment of a covalent bond between the surface and the molecule to be immobilized. Clean surfaces with hydroxyl groups were exposed with APTES. Silane groups present on this chemical compound react with the hydroxyl groups allowing the covalent binding of APTES onto the surface (Figure 4.1)

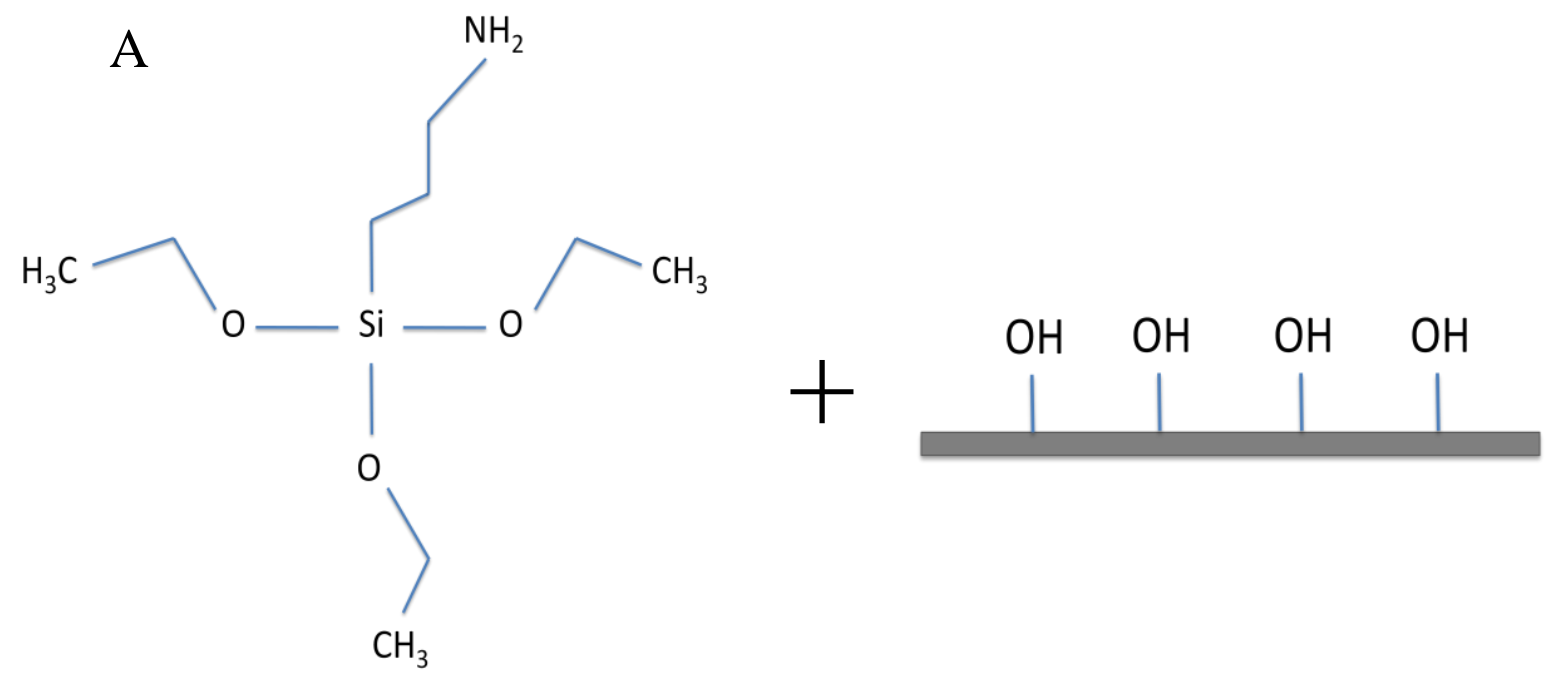


B

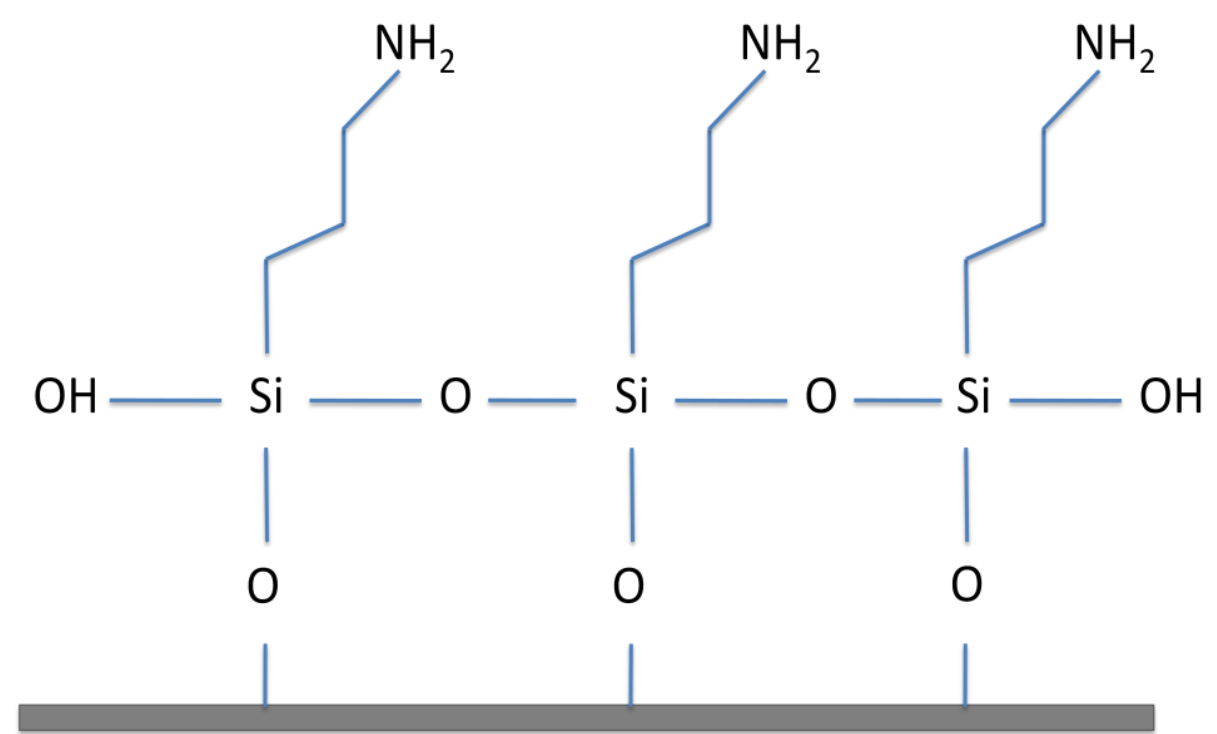

Figure 4.1 Functionalization of slides with amine groups A) APTES and glass prior to reaction B) Glass surface after silanization reaction

\subsection{Immobilization of Photobiotin}

Immobilization of photobiotin is achieved by the exposure of the compound to UV light of $365 \mathrm{~nm}$. The exposure produces structural changes on the aryl azide group of photobiotin eliminating nitrogen, which generates reactive, uncharged singlet or triplet nitrenes [63]. These nitrenes are especially reactive with $\mathrm{O}-\mathrm{H}$ or $\mathrm{N}-\mathrm{H}$ bonds. In this case nitrene radicals reacts with the available amine groups on the surface, establishing a covalent bond (Figure 4.2). 

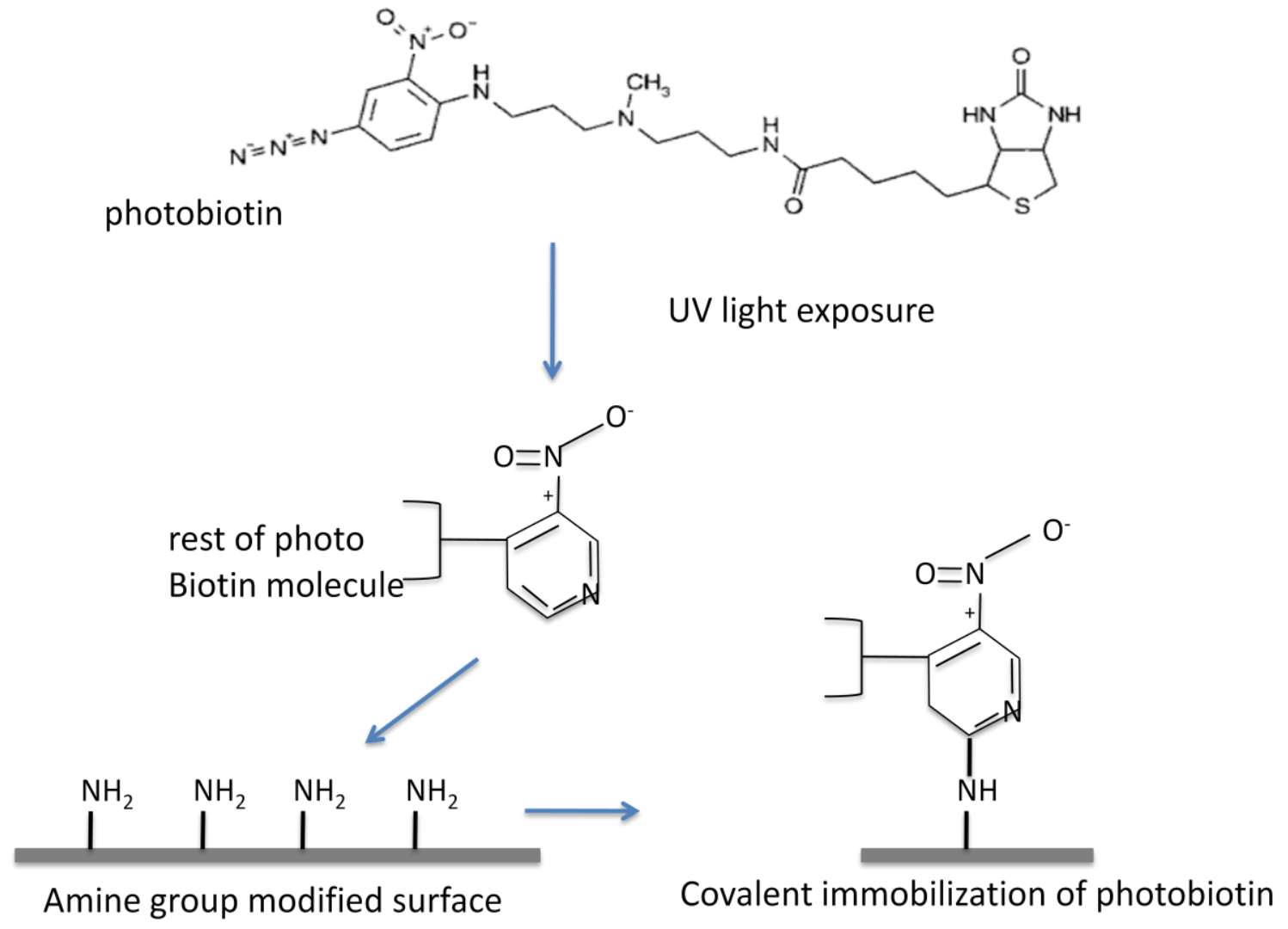

Figure 4.2 Immobilization of photobiotin on amine modified surface through activation of aryl azyde groups

\subsection{Immobilization of Fluorescent Streptavidin}

Immobilization of fluorescent-tagged streptavidin was used to determine the location of covalent immobilized photobiotin on the surface. The binding of these two molecules occurs through the bioaffinity of streptavidin (protein with four binding sites) to biotin (Figure 4.3) 


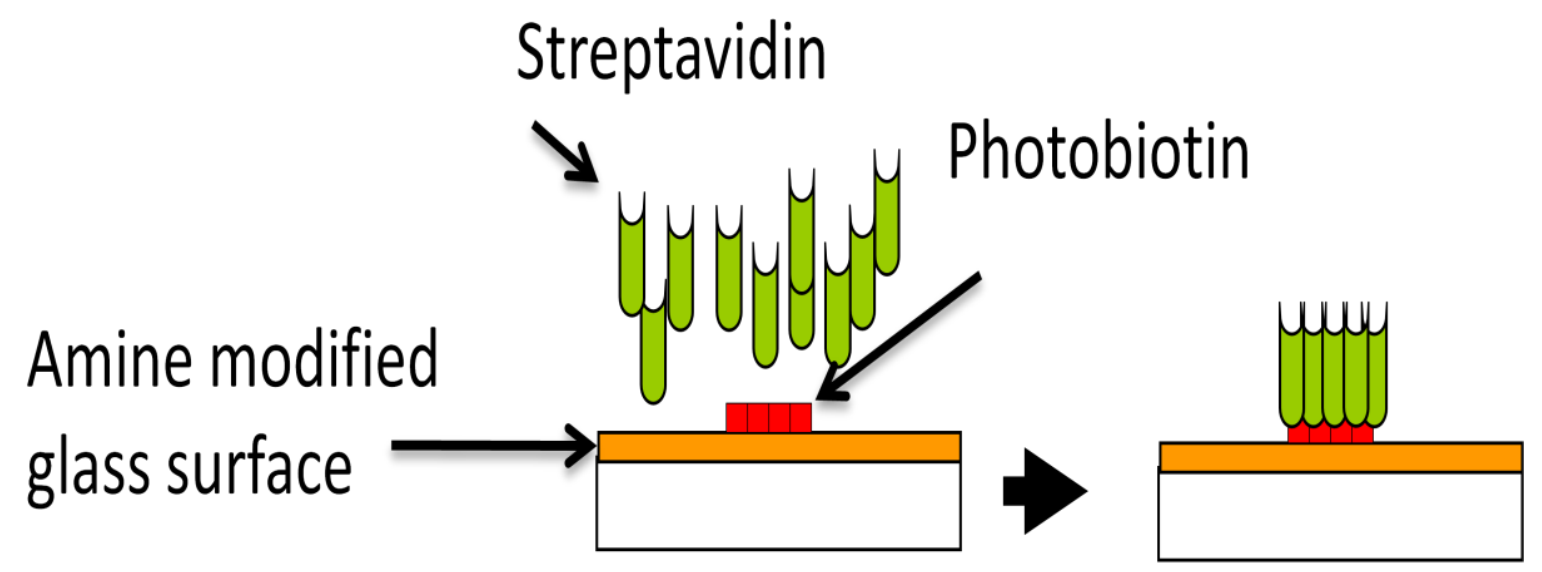

Figure 4.3 Binding of fluorescent streptavidin on patterned photobiotin via bioaffinity immobilization

\subsection{Conjugation of Actin with Biotinylated Gelsolin and Selective Immobilization of Actin}

Gelsolin is an actin binding protein which caps the fast growing end of the actin thereby controlling polymerization of the filaments. Gelsolin is conjugated with biotin and subsequently incubated with actin filaments. Biotin is used to help immobilize actin filaments by the specific capped end onto a streptavidin-coated surface (Figure 4.4). 


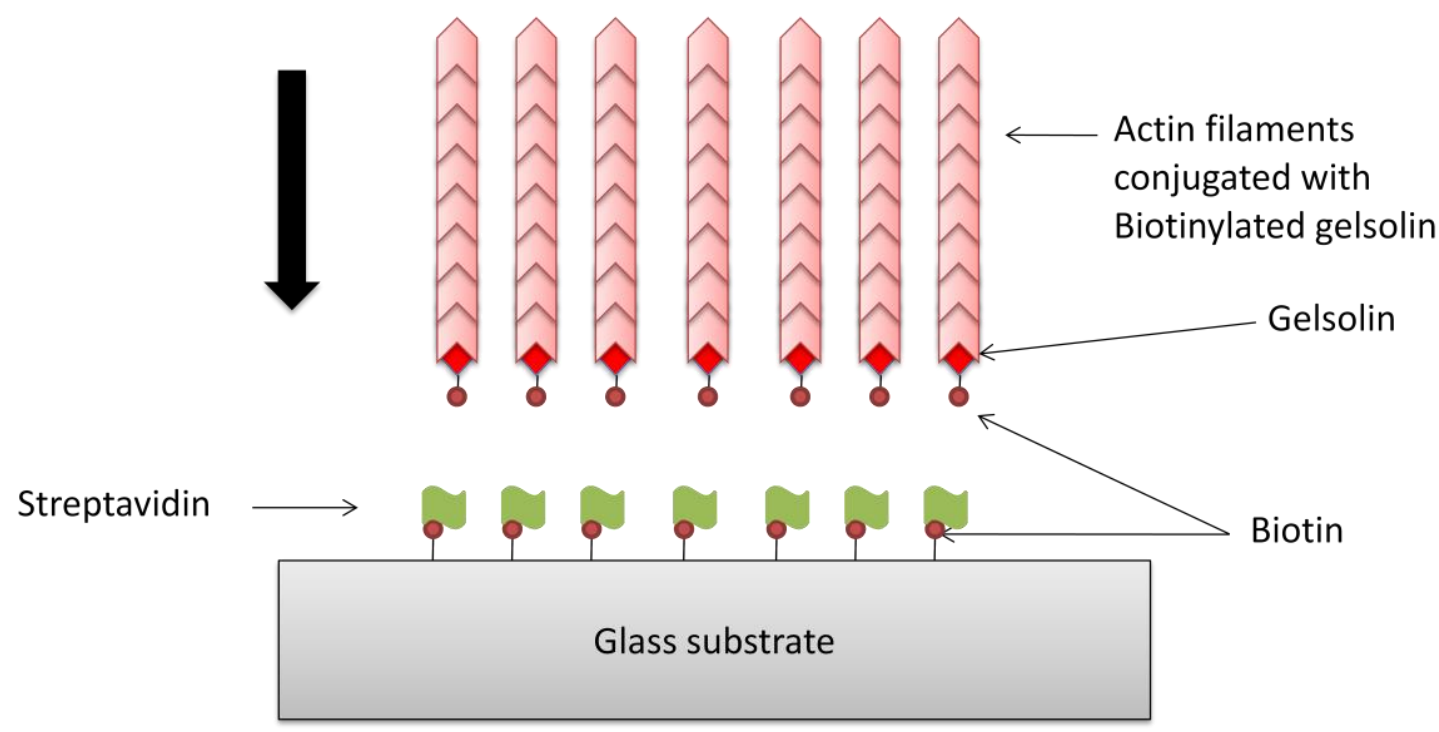

Figure 4.3 Cross sectional view of proposed selective attachment of actin filaments. Biotinylated gelsolin is conjugated with actin filaments and bound to the streptavidin coated areas via bioaffinity immobilization 


\section{CHAPTER 5 EXPERIMENTAL RESULTS}

In this chapter the methods used to characterize the patterns of actin filaments on protein coated surfaces are presented. The data analysis began with the characterization of the amino-silane groups using atomic force microscopy. The next step was to characterize the patterns by fluorescent imaging techniques, followed by the study of the influence of gelsolin on actin length and the study of blocking agents to prevent nonspecific binding of actin filaments. The final step was to characterize the binding of biotinylated-gelosin capped actin filaments in specific protein coated areas.

\subsection{Characterization of APTES Coated Slides}

Functionalization of glass slides with APTES was studied using atomic force microscopy (AFM). The main parameter measured in experiments was root mean square (RMS) roughness differences between surfaces. The starting point was to use AFM to characterize bare glass slides and compare the roughness values with the values obtained from the APTES coated slides. The system used for this imaging characterization was the MPF3D AFM by Assylum Research coupled with a computer running Igor pro version 6.0.4 software for data acquisition and image analysis. All AFM data reported here were conducted at room temperature.

Images of bare glass and APTES functionalized slides were taken using contact mode imaging. The AFM tips were selected according to the type of imaging modality to be used. The tips for tapping mode were primarily AC160TS (Olympus) with a spring constant of $42 \mathrm{~N} / \mathrm{m}$, a resonant frequency of around $300 \mathrm{kHz}$ and a tip radius of less than 
10nm (typical values around $7 \mathrm{~nm}$ ). For contact mode, AC240TS (Olympus) with a spring constant of $2 \mathrm{~N} / \mathrm{m}$ and tip radius between 7-11 nm as well as Veeco MSNL-10 with an spring constant of $0.03 \mathrm{~N} / \mathrm{m}$ and tip diameter of 2 to $3 \mathrm{~nm}$ were used. The preparation of both samples has been described previously in Chapter 3. The results obtained in these experiments suggested the presence of a monolayer of amino-silane groups on the glass slides treated with APTES. This conclusion is based on significant differences in roughness between clean glass slides and APTES-treated slides. APTEStreated slides show variable topography, demonstrating a nonconformal layer, the roughness of which differs from that of clean glass slides. Clusters of areas with greater roughness can be measured on the images and these aggregates can contribute to differences in overall surface roughness measured from modified and non-modified samples.

RMS roughness values for non-modified surfaces were obtained by placing glass slides on the AFM system and recording topographic information using contact mode imaging (Figure 5.1). 


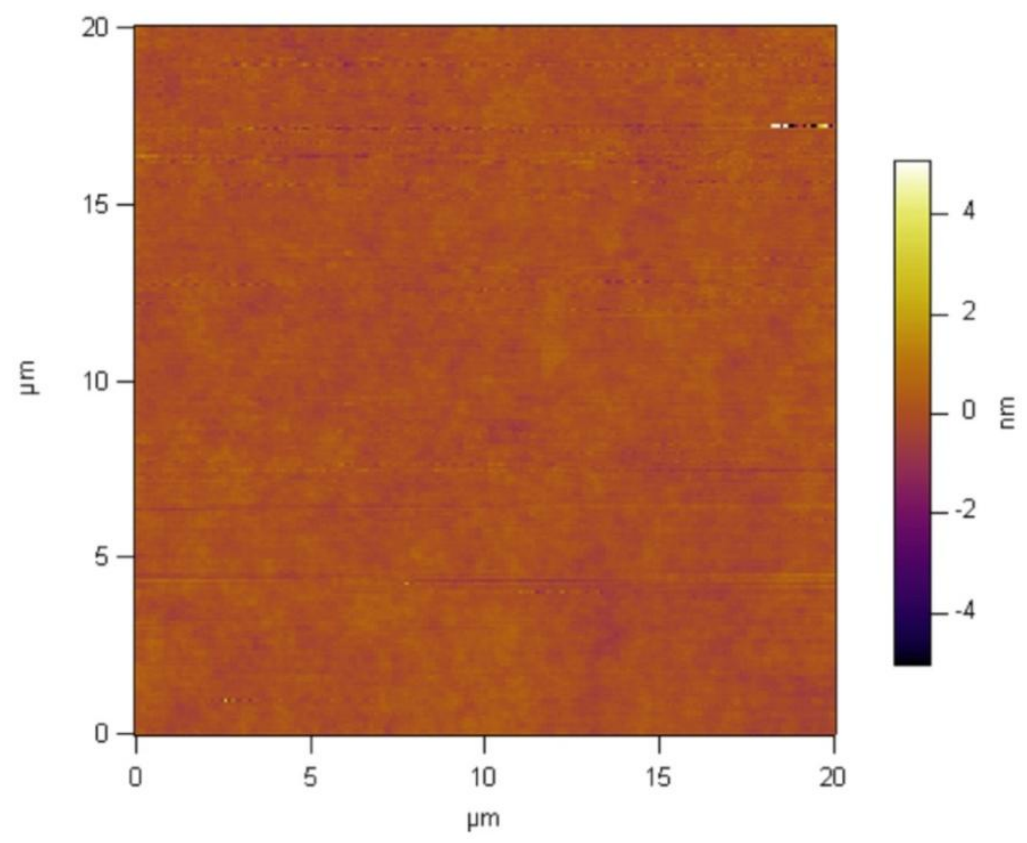

Figure 5.1 Contact Mode AFM image of a bare glass surface

Scanning of the samples was repeated several times. Multiple positions were scanned to provide a more complete profile of surface topography. The surface roughness was obtained after calculating the mean of 8 RMS roughness measurements obtained from the same sample. This "average" of RMS roughness measurements was determined to be approximately $0.245 \mathrm{~nm}$ for bare non-modified samples using contact mode imaging.

The same procedure was applied to APTES-functionalized glass slides. The roughness data was also averaged over 8 scans of the sample. Tapping and contact mode imaging were used in APTES-modified samples (Figure 5.2). 

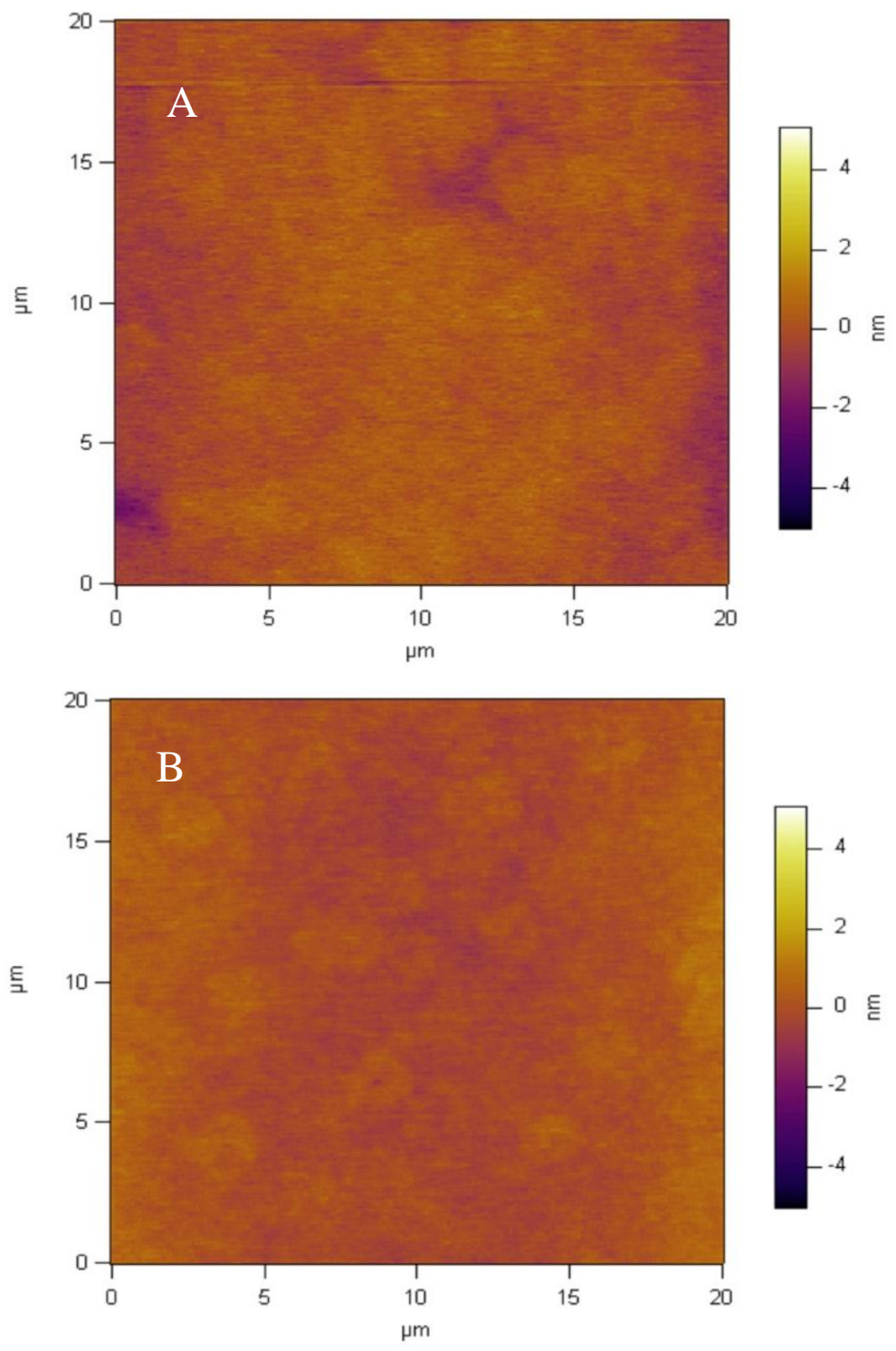

Figure 5.2 AFM images of APTES functionalized glass surfaces A) tapping mode B) contact mode.

The "average" of RMS roughness measurements obtained for contact mode was approximately $0.35 \mathrm{~nm}$, whereas for tapping mode was $0.4 \mathrm{~nm}$.

Relationship between roughness values and incubation time during APTES funcionalization of samples was also studied. Two incubation times were used during 
experiments; $0.5 \mathrm{hrs}$ and $12 \mathrm{hr}$ (Table 5.1). There were significant differences in roughness values in the samples suggesting that incubation time plays a role in the density of APTES deposited onto the surface. These findings were consistent with a previous report $[64,65]$. Other factors affecting the deposition of APTES include: silane coupling agent concentration in solution [64, 66, 67] and temperature [64].

\begin{tabular}{|l|c|}
\hline \multicolumn{1}{|c|}{ Image Modality (tapping) } & Surface Roughness (nm) \\
\hline $\begin{array}{l}\text { Incubation in 3\% APTES in Ethanol } \\
\text { for 0.5 hour }\end{array}$ & 0.4 \\
\hline $\begin{array}{l}\text { Incubation in 3\% APTES in Ethanol } \\
\text { for 12 hours }\end{array}$ & 0.81 \\
\hline
\end{tabular}

Table 5.1 Characterization results of APTES functionalization of glass slides with distinct incubation times.

\subsection{Patterning of Biotin on Amino-Silane Modified Glass Surfaces}

The patterns of photobiotin on the surface were determined by fluorescent imaging techniques. Streptavidin fluorescent-tagged protein was used to observe the photobiotin patterns on the amino-silane functionalized glass surfaces. The fluorescent characterization system consisted of a Nikon TE-200 Eclipse inverted microscope couple with a CCD camera for image acquisition along with Wasabi software (Hammatsu Photonics) installed in a PC computer. All the experiments were done in room temperature and the samples were maintain in aqueous solutions (PBS buffer, $\mathrm{pH}$ 7.2) to prevent protein denaturation and increase the lifetime of the fluorescent tag of the protein. 
The data recorded showed correspondence between the fluorescence observed on the recorded image with the geometrical shapes printed on the photofilm mask used during the UV irradiation of photobiotin. Figure 5.3 shows a fluorescent image, the corresponding pattern drawn on the photofilm mask as well as intensity profile. The concentration of streptavidin and photobiotin was $0.8 \mathrm{mg} / \mathrm{ml}$ and $0.1 \mathrm{mg} / \mathrm{ml}$ respectively.
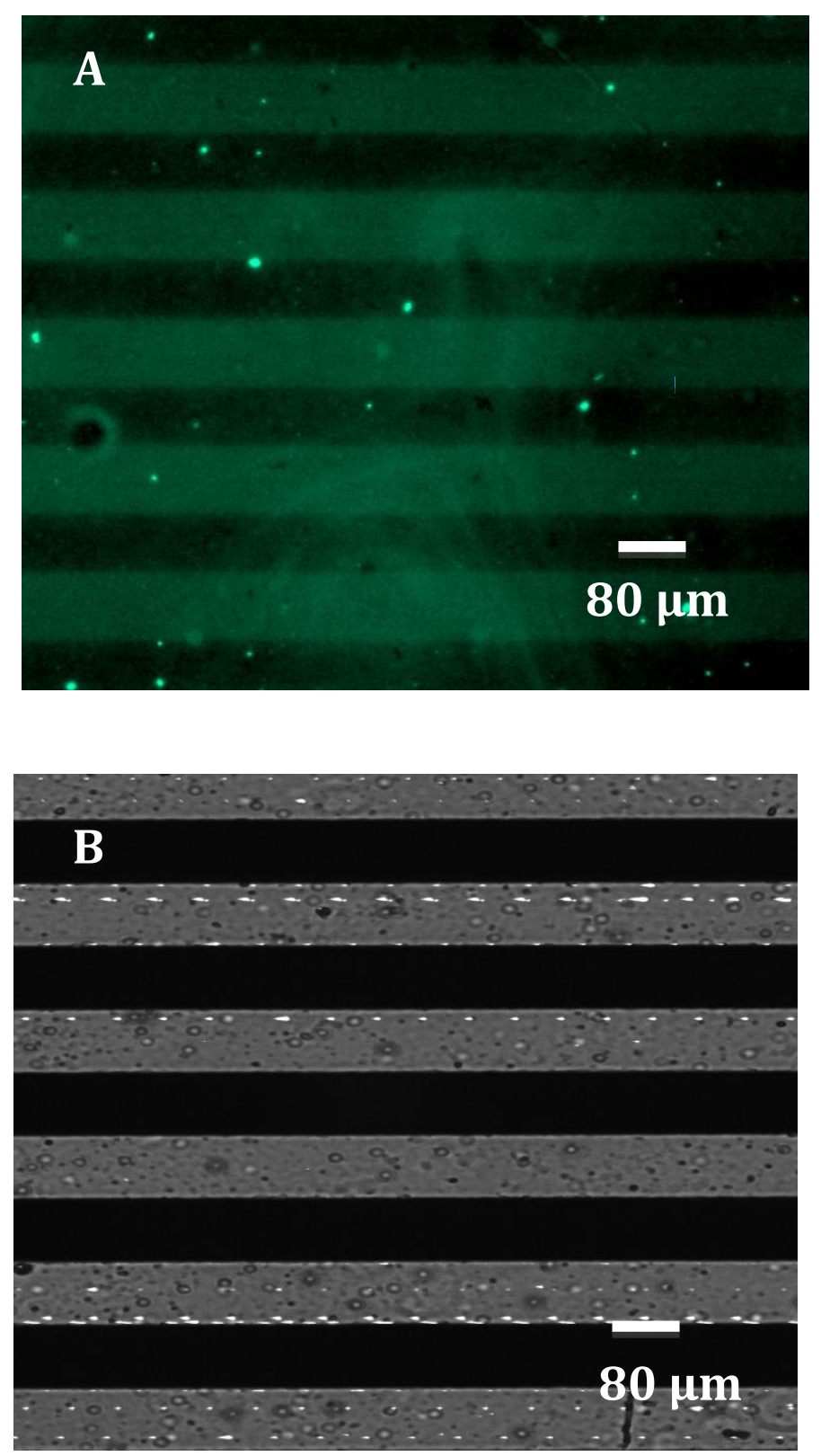


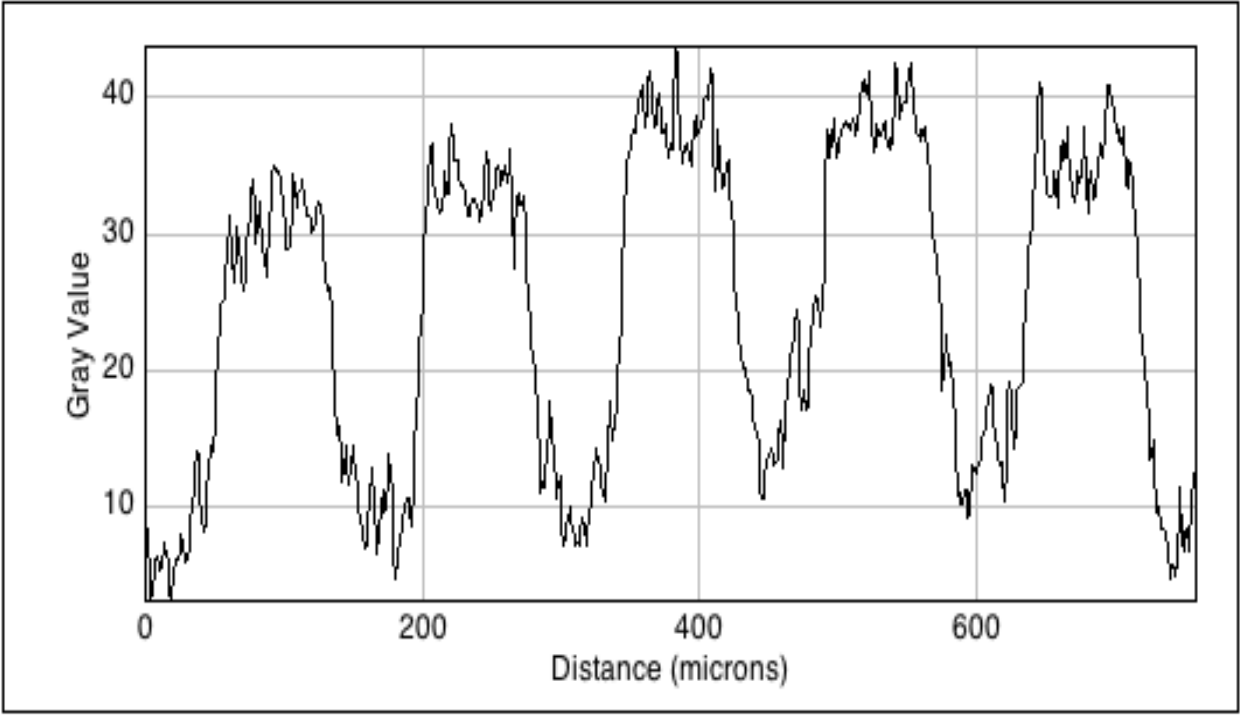

Figure 5.3. Fluorescent characterization of protein patterning A) Fluorescent microscopy image of $80 \mu \mathrm{m}$ thick line features B) microscopy image of corresponding photofilm mask $\mathrm{C}$ ) intensity profile plot

Photofilm masks with different types of geometrical patterns were used to assess the reproducibility of this method (Figure 5.4). 

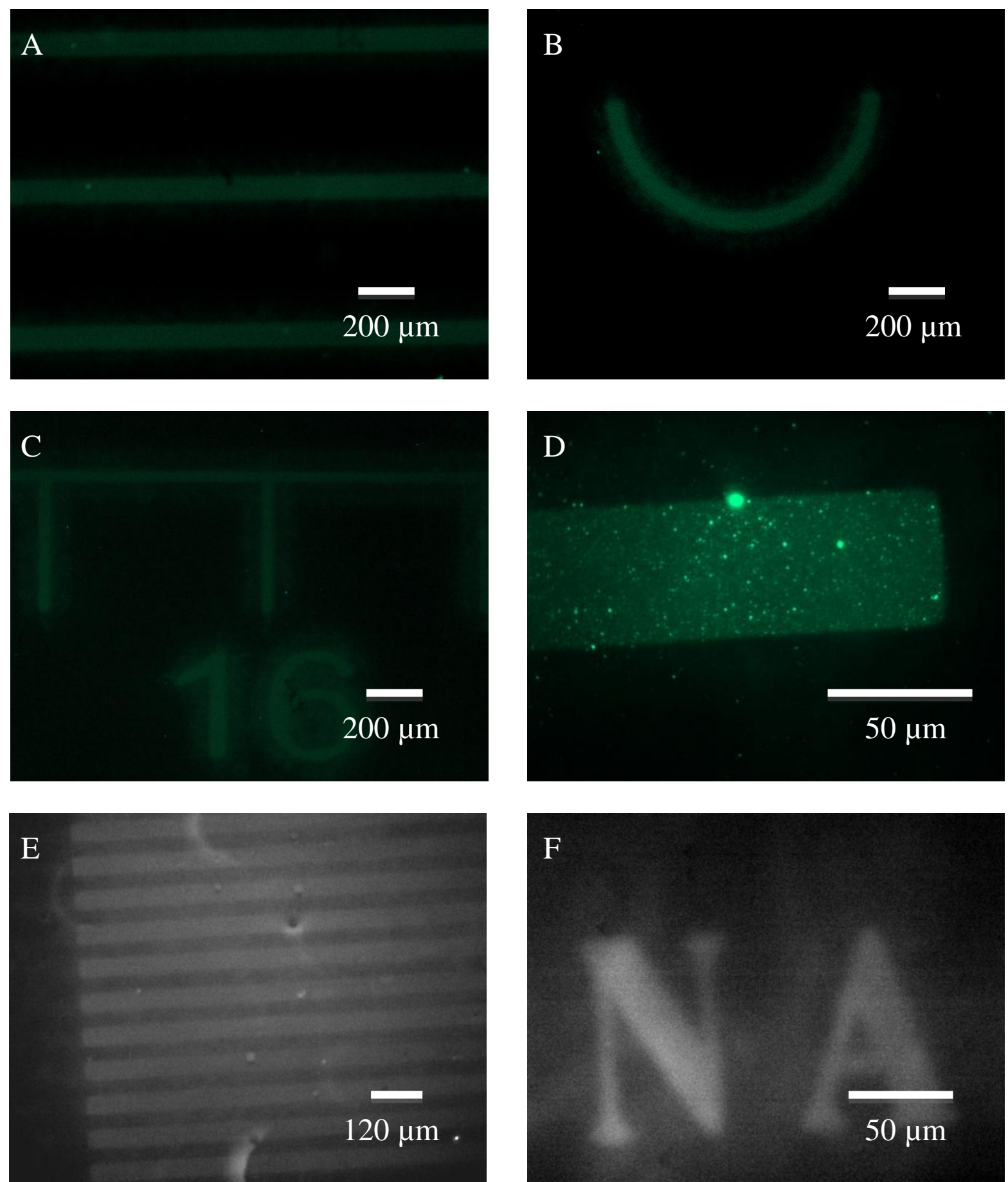

Figure 5.4. Fluorescent images of patterned proteins with distinct geometries A) 80 $\mu \mathrm{m}$ thick line features B) $70 \mu \mathrm{m}$ curve feature C) patterned numbers D) edge of a 50 $\mu \mathrm{m}$ thick line feature $\mathrm{E}) \mathbf{3 0} \boldsymbol{\mu m}$ thick line features $\mathrm{F}$ ) patterned letters. 


\subsection{Fluorescent Intensity-Streptavidin Concentration Correlation}

Experiments were conducted to find the correlation between the fluorescent intensity and the concentration of fluorescent-tagged streptavidin bound to the patterned photobiotin. The procedure to calculate the intensity values is done using the image analysis tools on Wasabi. A region of interest (ROI) box was defined and placed on the acquired images and total summation of pixel intensity values as well as mean, maximum and minimum values of pixel intensities within the enclosed region were obtained (Figure $5.5)$.

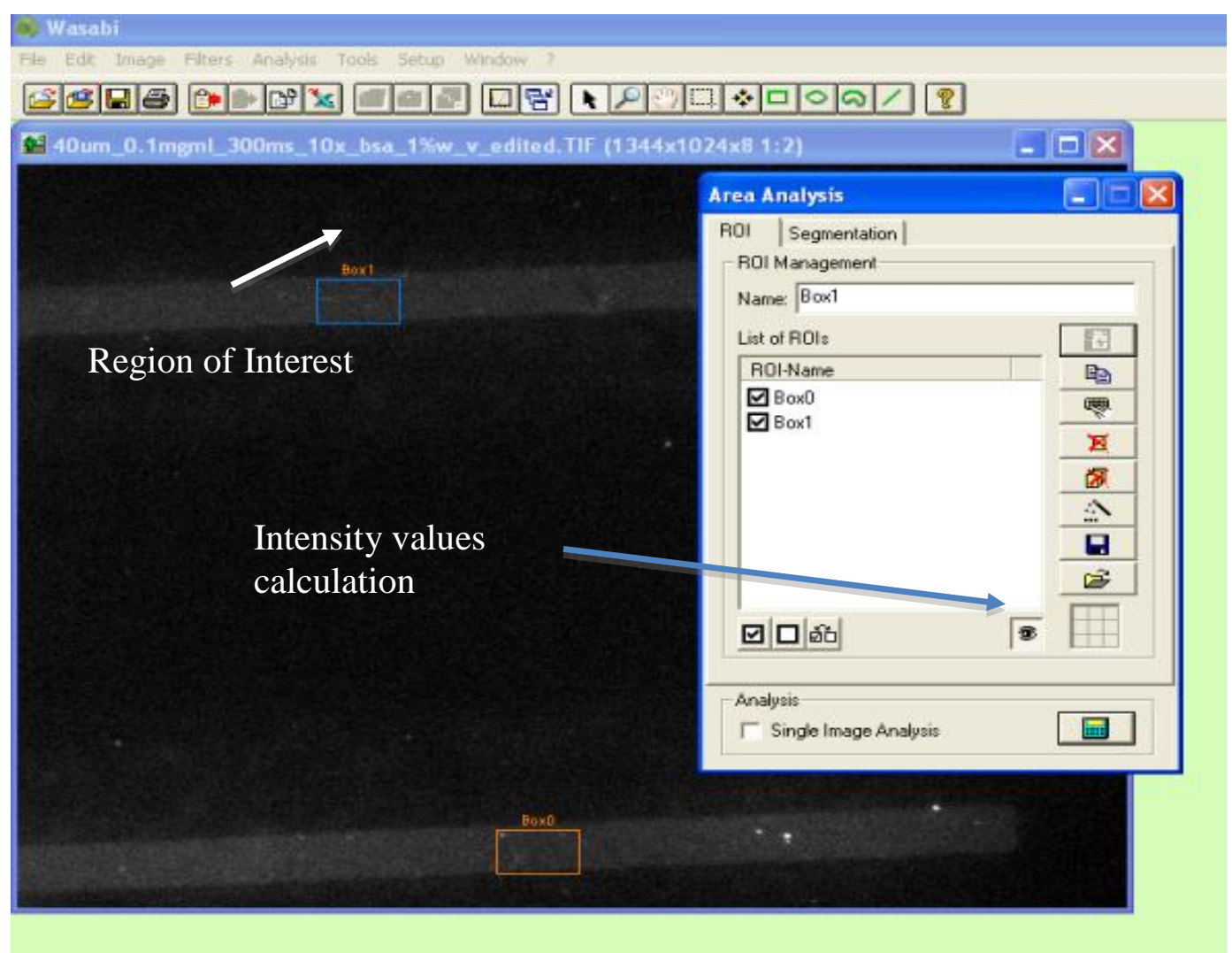

Figure 5.5 Calculation of pixels intensity values in the region of interest using Wasabi

Concentrations of FITC streptavidin, which ranged from $0.04 \mathrm{mg} / \mathrm{ml}$ to $0.8 \mathrm{mg} / \mathrm{ml}$ were used in this study. 
All image acquisition parameters, such as time of exposure, gain, contrast and LUT values were kept constant for all the images to ensure consistency during the intensity measurements. The software allowed saving all the measurements into .xls files, which later were plotted using Microsoft Excel. Results of these measurements showed a positive correlation between fluorescent intensity and concentration of streptavidin (Figure 5.6). This data indicated that total fluorescent intensity values increase as the concentration of fluorescent-tagged protein increases. Results also demonstrate that there is a saturation point at a concentration of approximately $0.4 \mathrm{mg} / \mathrm{ml}$. This signifies that at $0.4 \mathrm{mg} / \mathrm{ml}$, almost all biotin binding sites are occupied by streptavidin.

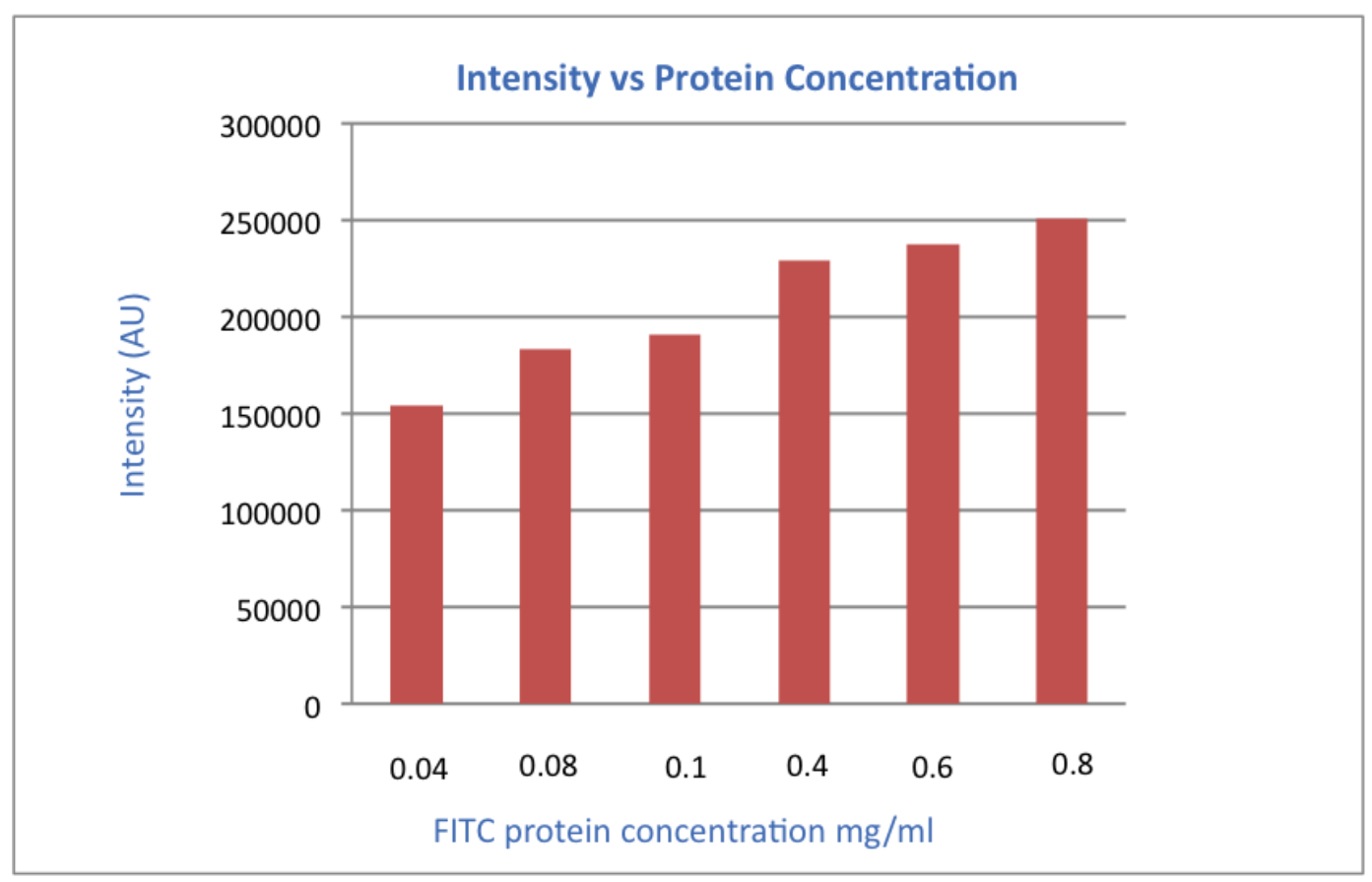

Figure 5.6 Intensity vs. concentration of streptavidin on patterned surfaces 


\subsection{Protein Patterning by Microcontact Printing}

Microcontact printing for selective micropatterning of protein was also explored. This method was simple to perform and served as a comparison to the photoimmobilization method. Patterns of FITC streptavidin with a concentration of $1 \mathrm{mg} / \mathrm{ml}$ were accomplished over clean glass substrate after incubating a PDMS stamp with fluorescent-tagged proteins and applying mechanical force to transfer the patterns to the surface (Figure 5.7 (a)). The fluorescent inverted microscope was used to assess the presence of patterns. This method was not further used because of problems of reproducibility of the patterns (patterns were not uniform, particularly for features bellow $10 \mu \mathrm{m}$ ) and protein stability (Figure 5.7 (b)). The photoimmobilitation strategy, however, is stable even after various washing processes due to the strong bond formed between biotin and streptavidin and the even stronger bond (covalent) between biotin and the amino-silane functionalized surface. Also, there was the important issue of losing protein functionality after immobilization on the surface, which commonly occurs with microcontact printing.

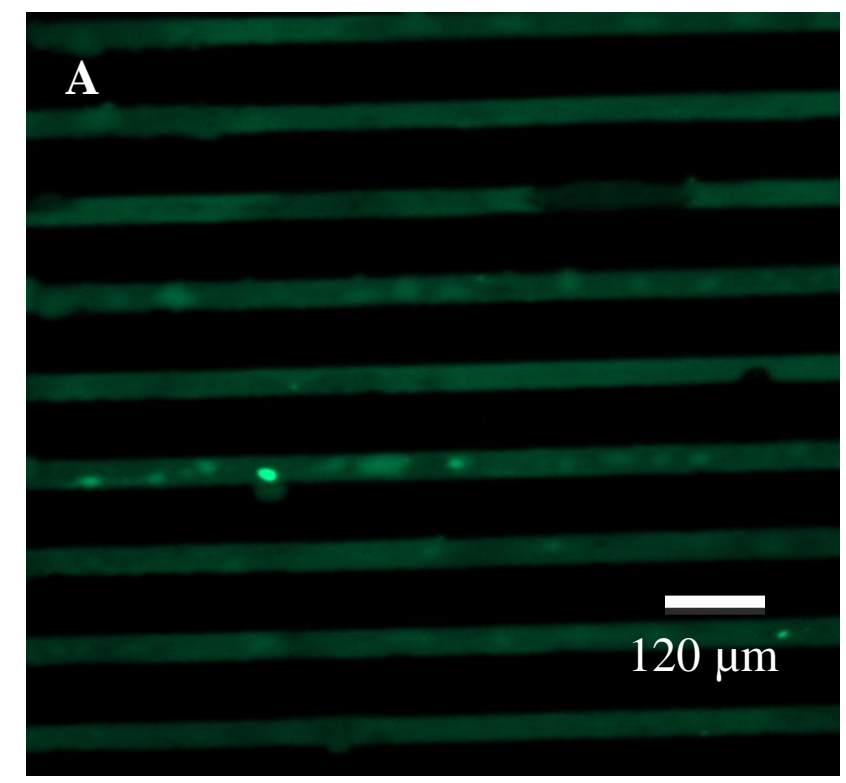



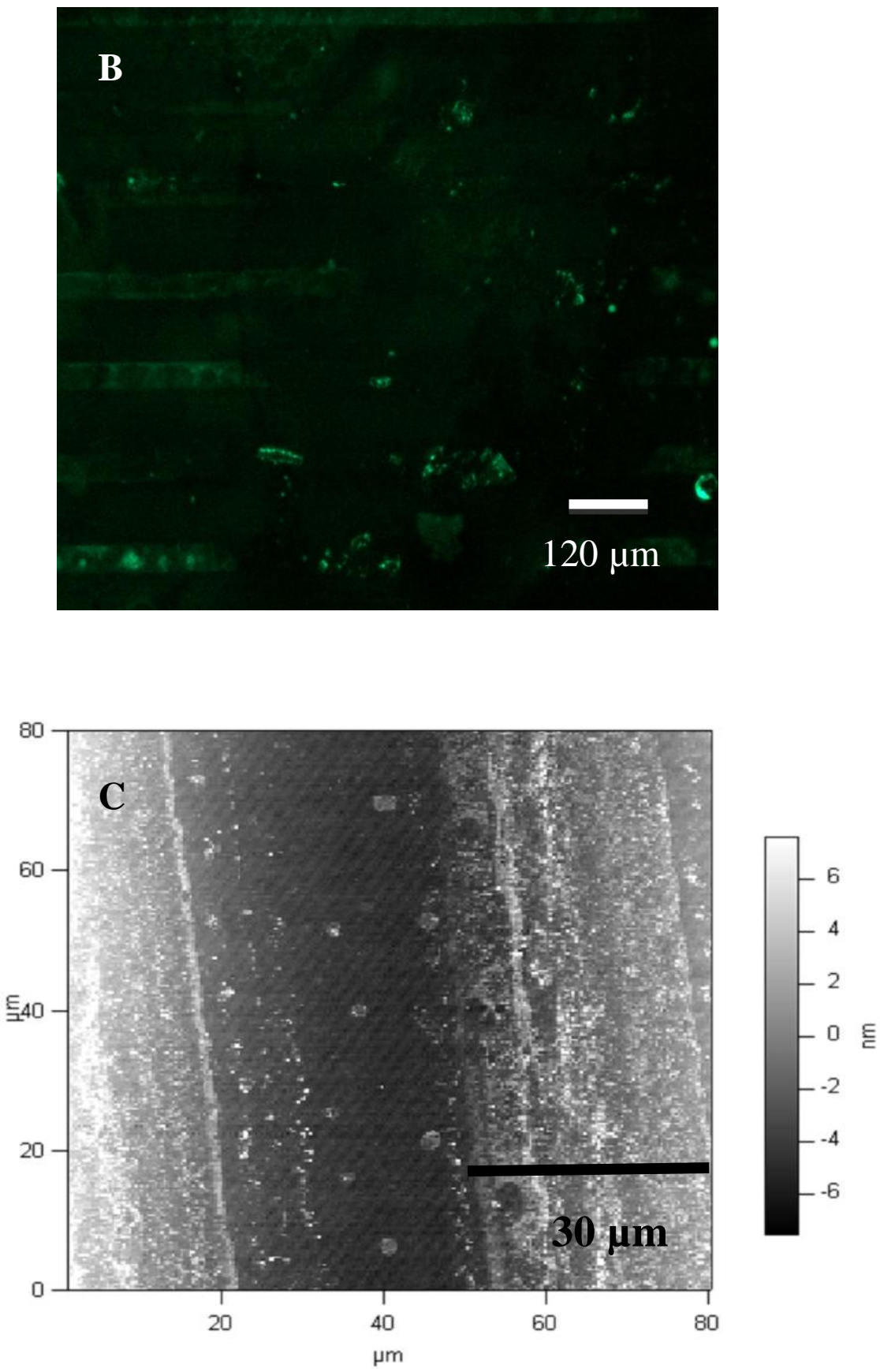

Figure 5.7 Microcontact printing of streptavidin. A) $30 \mu \mathrm{m}$ thick line features B) image of a sample after 3 washes in PBS buffer ( 5 minutes each) C) tapping mode AFM images of the patterned proteins (30 $\mu \mathrm{m}$ thick line features). 


\subsection{Optimization of Actin Length for Attachment to Biotin-Patterned}

\section{Surfaces}

In this section the relationship between concentration of gelsolin and the length of the actin filaments were studied to find a proper length to be used during the immobilization of actin filaments onto protein coated surfaces. Determination of actin length was achieved by applying an automatic tracking procedure using Image J.

A photofilm mask with features of known sizes $(80 \mu \mathrm{m}$ thick line features) was used as a reference in the microscope to allow the transformation of the field of view of the recording system from pixels dimensions (1344 (horizontal) x 1024 (vertical)) into equivalent dimensions in microns (Figure 5.8).

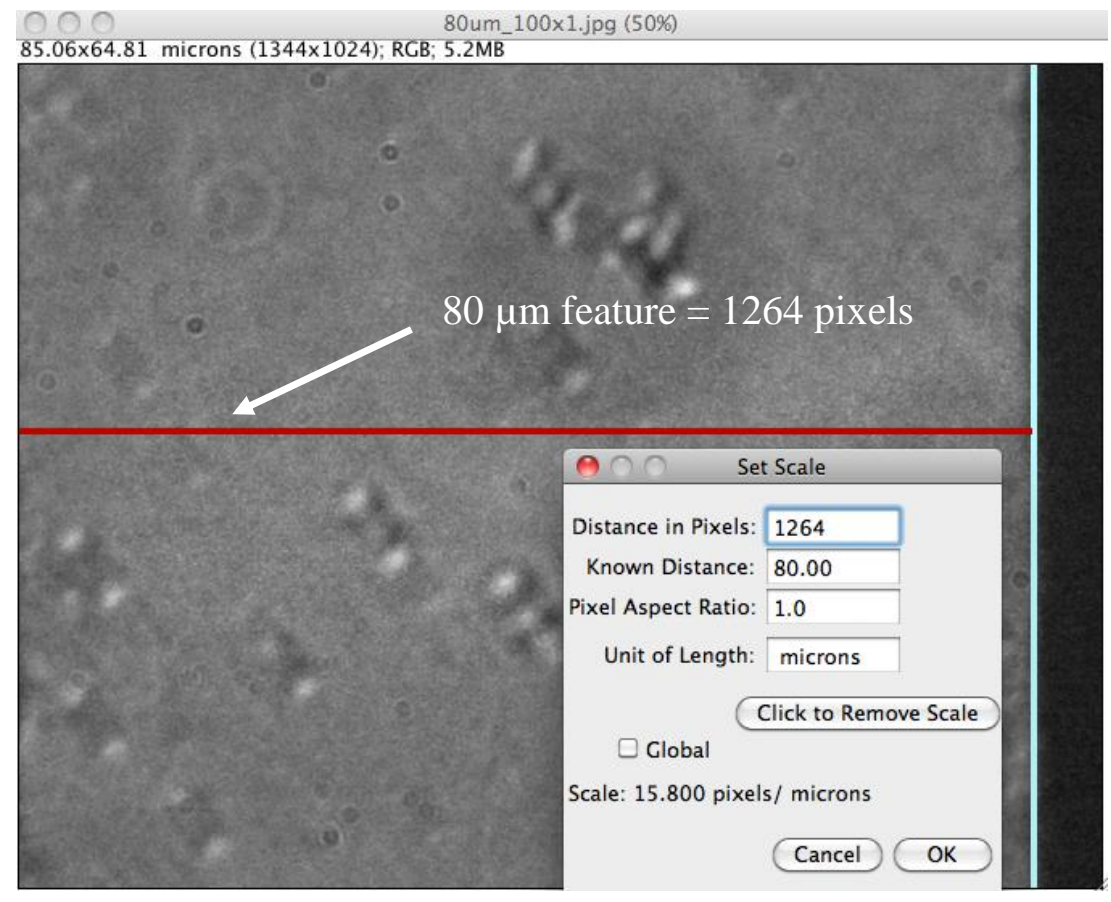

Figure 5.8 Determination of field of view in micron dimensions 
A mask feature was then measured in pixels dimension and this value along with the known dimensions in microns were entered in the "set scale" feature of image J, resulting in a window in micron dimensions of $85.06 \times 64.81$, which is equivalent to 15.8 pixels/micron.

Three different concentrations $(1.23 \mu \mathrm{g} / \mathrm{ml}, 0.123 \mu \mathrm{g} / \mathrm{ml}$ and and $0.0123 \mu \mathrm{g} / \mathrm{ml})$ were used during the experiments to find the relationship between the concentration of gelsolin and the length of the filaments. The resulting images were processed with image $\mathrm{J}$ and the length of filaments determined for each of the samples. Filaments with lengths greater or less than two standard deviations of the average length were excluded from analysis (Figure 5.9). These results showed an inverse relationship between the concentration of gelsolin and the length of actin filaments (Figure 5.10).

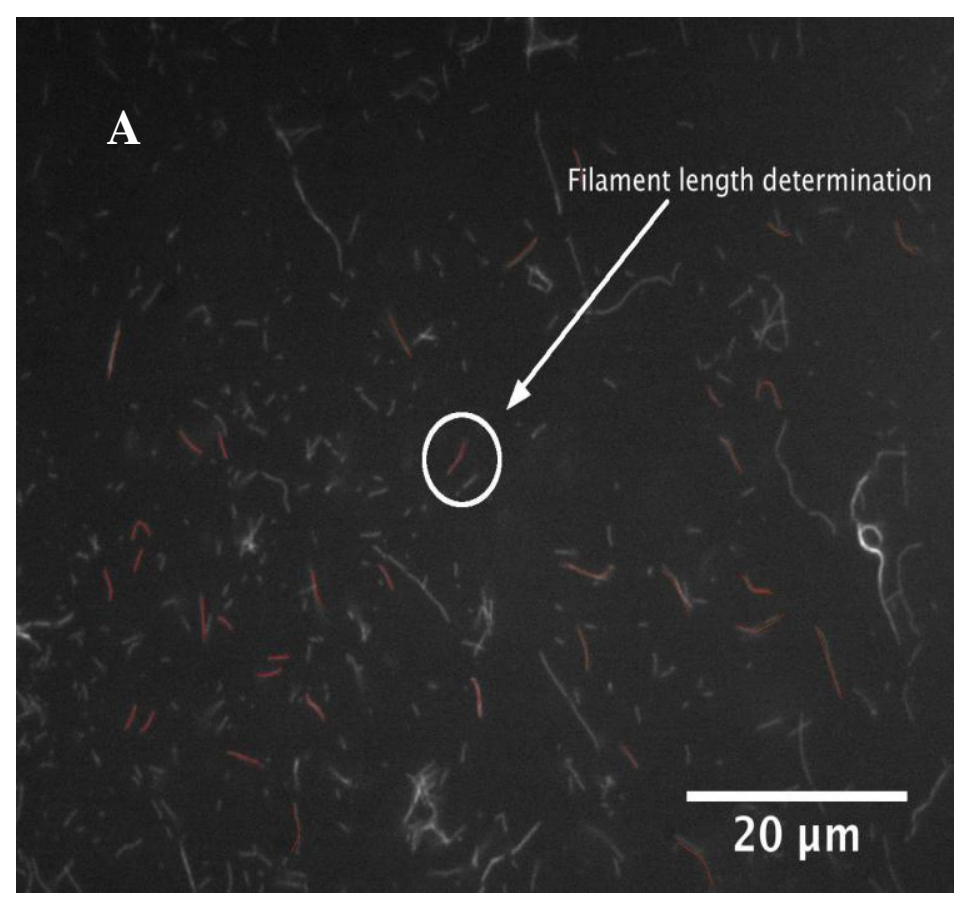



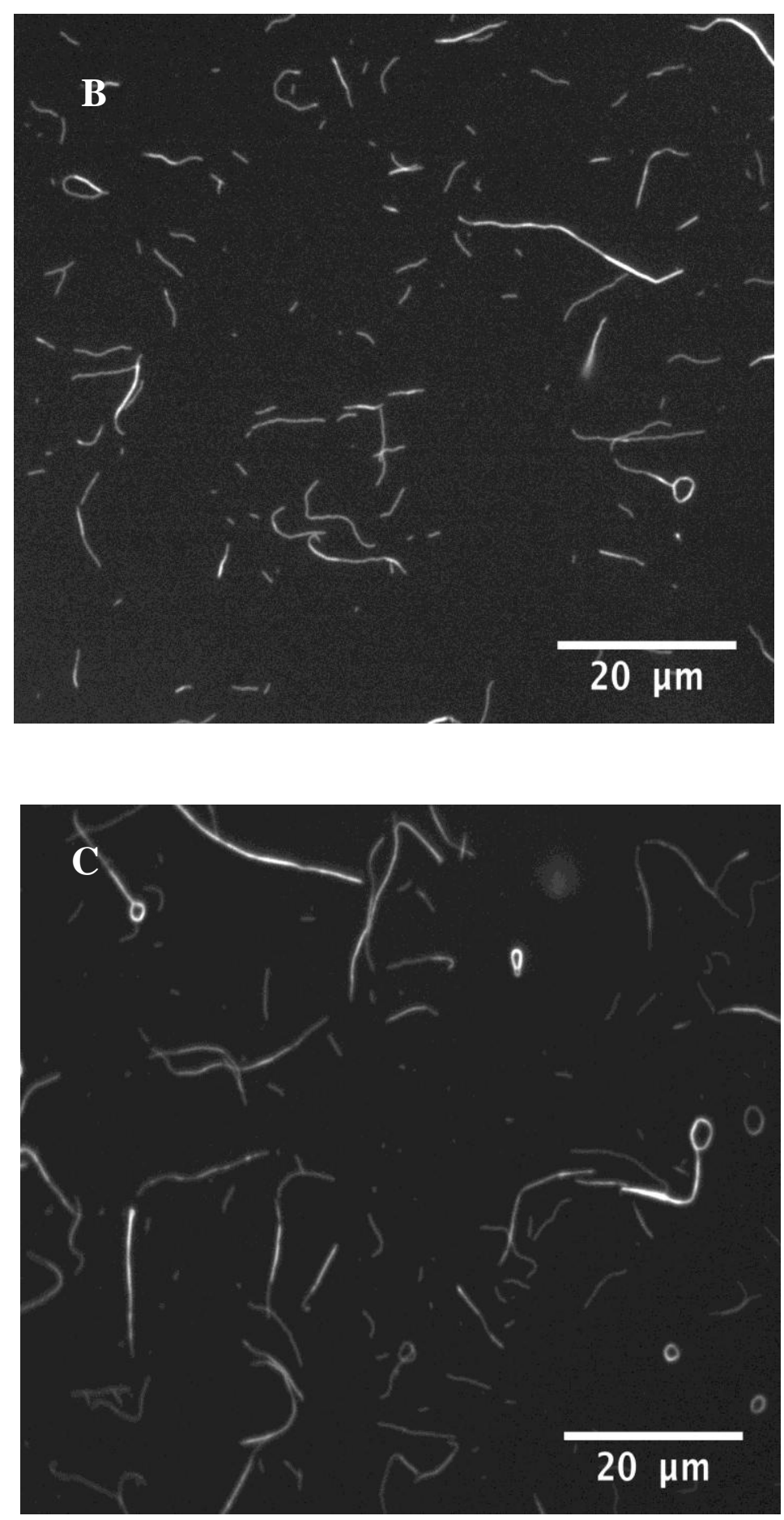

Figure 5.9 Actin filaments length calculation at different gelsolin concentrations. A) $1.23 \mu \mathrm{g} / \mathrm{ml} \mathrm{B)} 0.123 \mu \mathrm{g} / \mathrm{ml} \mathrm{C)} 0.0123 \mu \mathrm{g} / \mathrm{ml}$ 


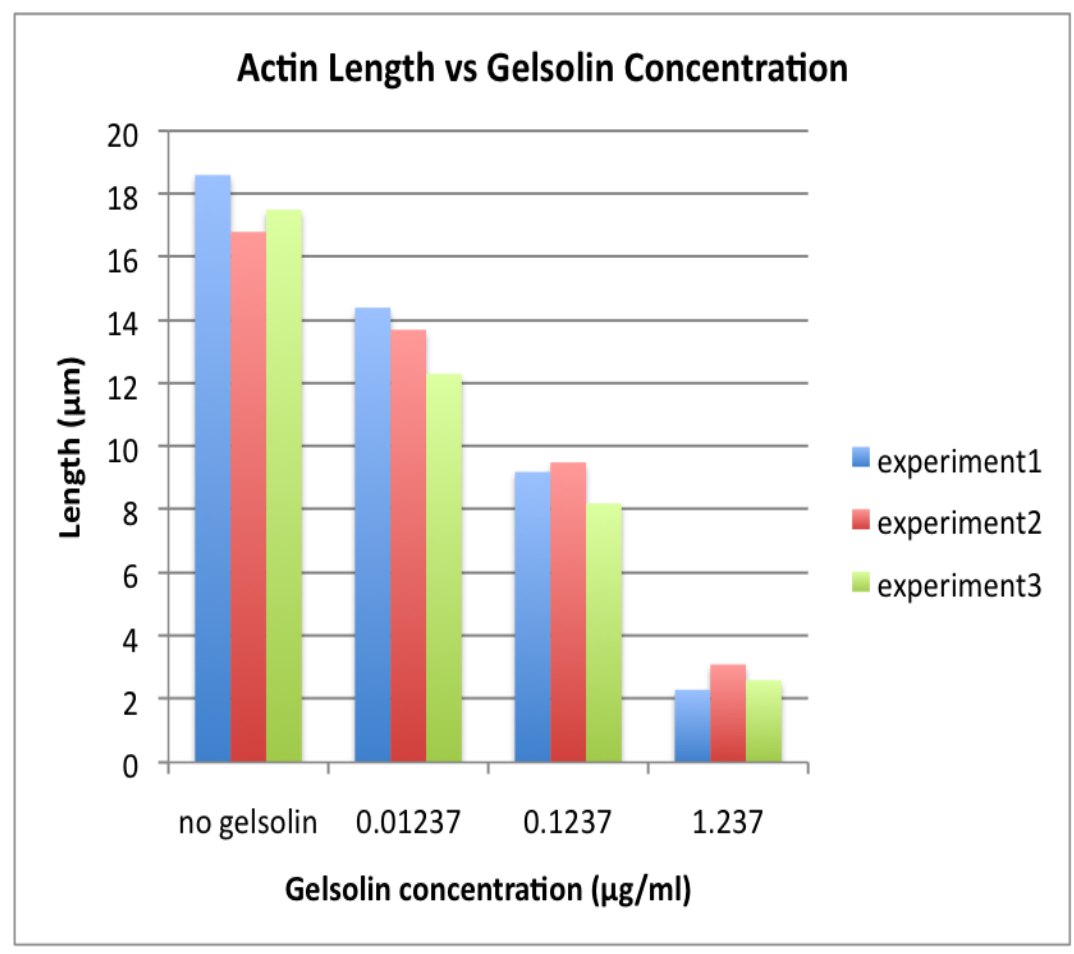

Figure 5.10. Relationship between actin filament length and gelsolin concentration.

\subsection{Characterization of Non-Specific Binding of Actin}

Another aspect studied during experiments was the control of non-specific binding of protein on the surface. Experiments were conducted using three types of blocking agents; Tween20, BSA and NHS-polyethylene glycol (MS-PEG, Pierce). Control experiments were done to test the effectiveness of these blocking agents in the presence of actin filaments without gelsolin (ideally all the filaments should be washed out). The following list describes the experiments conducted including control experiments:

1) BSA on APTES coated slides ( 0.5 and 12 hours incubation time) + actin filaments (control experiment). 
2) BSA on APTES + photobiotin + streptavidin coated surface + actin filaments NHS- Polyethylene glycol on APTES coated slides (APTES slides functionalized during 0.5 and 12 hours) + actin filaments.

3) NHS- Polyethylene glycol on APTES (APTES slides functionalized during 0.5 and 12 hours) + actin filaments (control experiment).

4) NHS- Polyethylene glycol on APTES + photobiotin + streptavidin + actin filaments

5) Tween 20 on APTES + actin filaments (control experiment)

Images were analyzed with image $\mathrm{J}$, and the number of filaments present in each of the samples was counted.

The same scale procedure used to convert the dimension from pixels to microns described in section 5.5 was implemented for this study. The image was preprocessed by a thresholding function that allowed the selection of the filaments to be counted. Figure 5.11 shows a preprocessed image by thresholding with the subsequent calculation of the number of filaments.

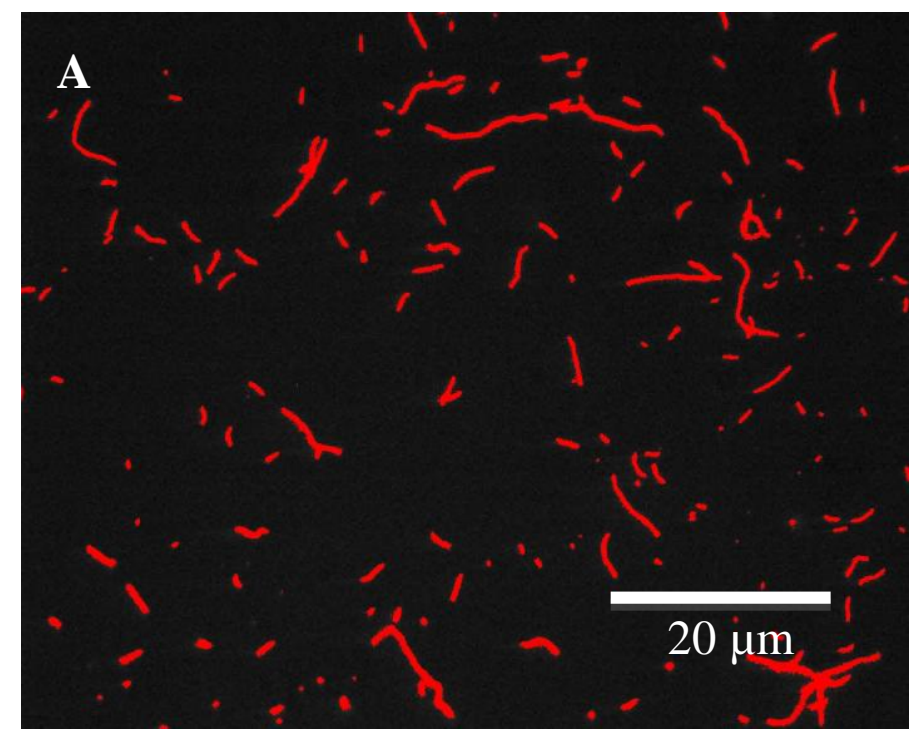




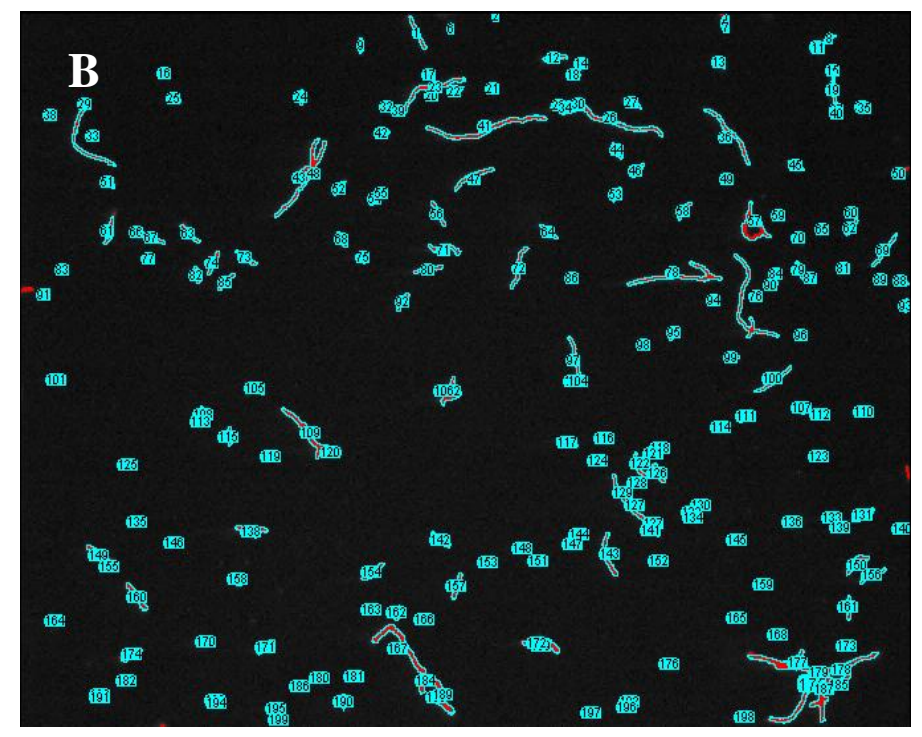

Figure 5.11 Actin filaments counting A) Thresholded image B) Calculation of number of filaments in the image (each blue label represents a filament, MS-PEG was the blocking agent)

Results indicate that BSA had the greatest ability to prevent non-specific binding of actin filaments. Non-specific binding experiments showed a significant non-specific binding of actin filaments in the MS-PEG blocked surfaces compared to surfaces blocked with BSA (Figure 5.12).

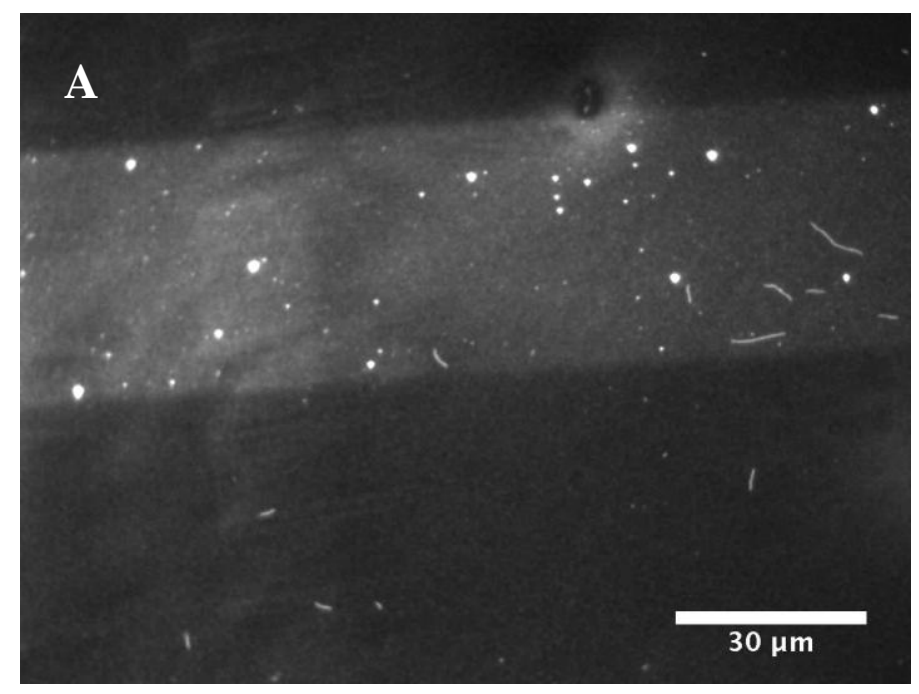




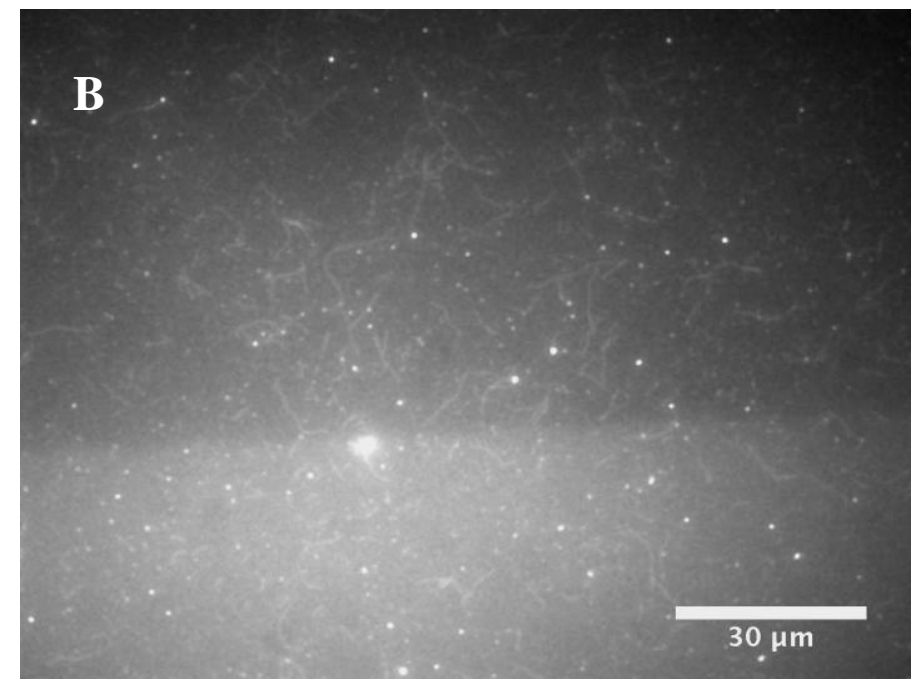

\section{Figure 5.12 Comparison between blocking agents in protein patterned samples A) $3 \% \mathrm{v} / \mathrm{v}$ BSA B) $2.5 \mathrm{mM}$ of MS-PEG}

NHS-Polyethylene glycol showed significant blocking ability (only during control experiments with glass slides that were functionalized in APTES solution for 12 hours). Tween-20 did not work in any of the experiments involving actin, although it worked well during the photoimmobilization of streptavidin.

In summary, the results obtained by comparing blocking agents demonstrated that BSA had the greatest ability to prevent non-specific binding of actin filaments in all types of experiments (Figure 5.13). The likely reason is the size of BSA (molecular weight: 66,000 Da, [68]) in comparison to other chemical elements such as MS-PEG (molecular weight: $333 \mathrm{Da}$ for MS-(PEG) $\left.{ }_{4}[69]\right)$. BSA can cover more effectively the active sites (increasing the rejection to proteins in these areas) except the occupied sites by streptavidin. In addition, $\mathrm{BSA}$ isolectric point $(\mathrm{pI})$ is $\mathrm{pH} \sim 4.7[70,71]$ so, at neutral $\mathrm{pH}$, BSA contains a net negative charge (similar to actin, pI $\sim 5.5,[72]$ ), thereby creating electrostatic attraction between these proteins and the surface and leading to more 
complete coverage of the positively charged amine-functionalized surface. NHSpolyethylene glycol provides good blocking only with APTES slides incubated for longer time, which may be explained by how the NHS group binds to the amino-silane groups, which could lead to empty spaces where actin can bind. Finally, Tween 20 did not show significant blocking for any experiments with actin filaments. Tween 20 is a low ionic strength detergent that can be used as a blocking agent for specific cases. In these studies Tween 20 was used to wash and block non-specific binding of streptavidin as well as for actin. Streptavidin is near neutral at $\mathrm{pH} 7$, while actin is negatively charge. These charge differences between actin and streptavidin at neutral $\mathrm{pH}$ explain why Tween 20 worked well for photochemical immobilization of streptavidin but not for experiments involving actin filaments

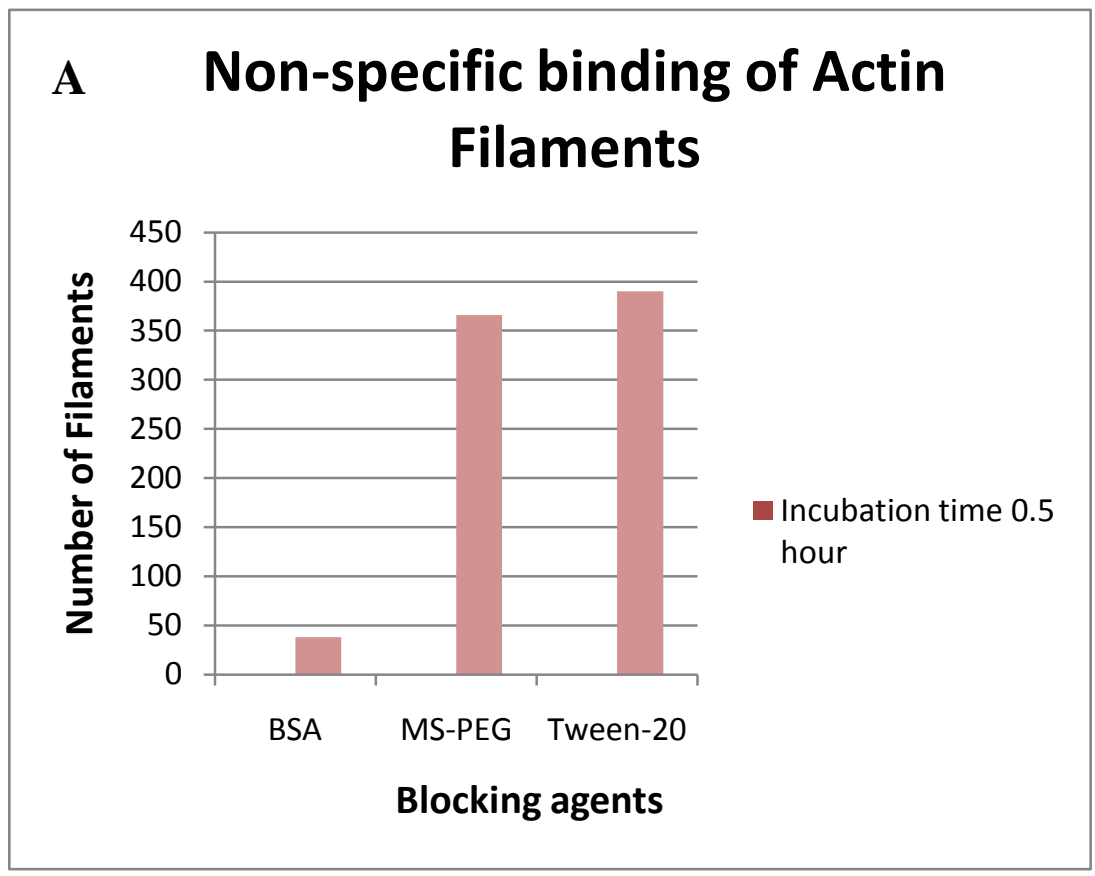



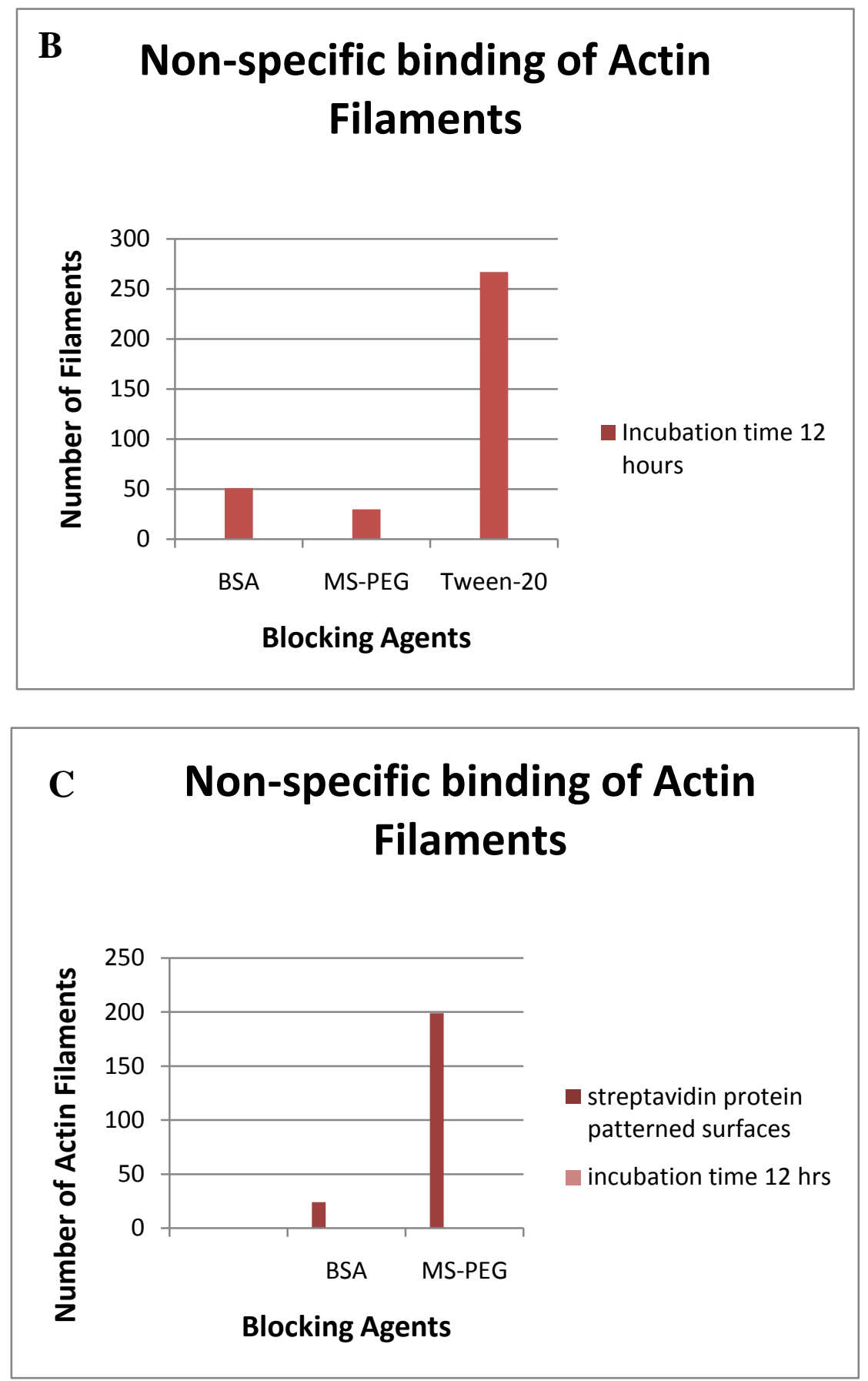

Figure 5.13 Non-specific binding blocking agents experimental results A) $3 \%$ v/v BSA, 2.5mM MS-PEG and 1\% v/v Tween-20 on functionalized slides incubated in APTES for $0.5 \mathrm{hr}$ B) $3 \% \mathrm{v} / \mathrm{v}$ BSA, 2.5mM MS-PEG and 1\% v/v tween-20 in functionalized slides incubated in APTES for $12 \mathrm{hr} \mathrm{C)} 3 \% \mathrm{v} / \mathrm{v} \mathrm{BSA,} \mathrm{2.5mM} \mathrm{MS-}$ PEG in protein patterned surfaces. All measurements were taken in windows with dimension of $139.64 \mu \mathrm{m} \times 106.39 \mu \mathrm{m}$. 


\subsection{Attachment of Actin on Streptavidin Patterned Surfaces}

The final study of this thesis was to attach biotinylated gelsolin-capped actin filaments to streptavidin coated areas. The procedure to track the results was similar to previous experiments (sections 5.5 and 5.6) using Image $\mathrm{J}$ to track the quantity of filaments attached to the streptavidin areas and compare this value to the number of filaments attached to the background (non-streptavidin coated areas). The experiment was conducted following the procedures described previously in Chapter 3 (patterning of streptavidin by photoimmobilization of photobiotin followed by incubation with biotinylated-gelsolin capped actin filaments) (Figure 5.14).

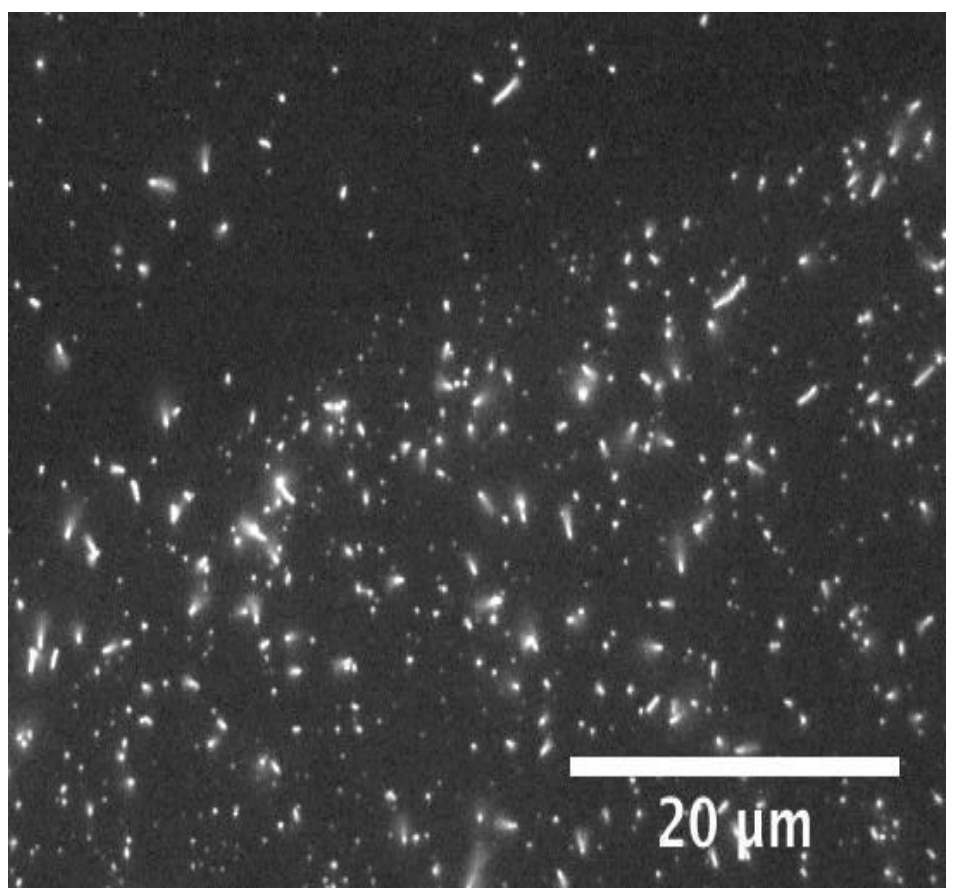

Figure 5.14 Patterning of actin on streptavidin coated surfaces. 
Pressure driven flow was used to lay down the actin filament on the surface to determine if biotinylated actin was immobilized on streptavidin-coated areas. There were two ways used to characterize the attachment of actin filaments; direct counting of the number of filaments in both areas (protein coated and background), and by using two distinct images one before and one after pressure driven flow was applied. These two images were then subtracted and the number of filaments from the resulting image was determined by the Image J "analyzed particles" function.

The images from experiments were extracted from recorded AVI videos and thresholded as a previous step to automatically obtain the number of filaments. Figures 5.15 and 5.16 show the thresholded images and the subsequent calculation of the number of filaments.

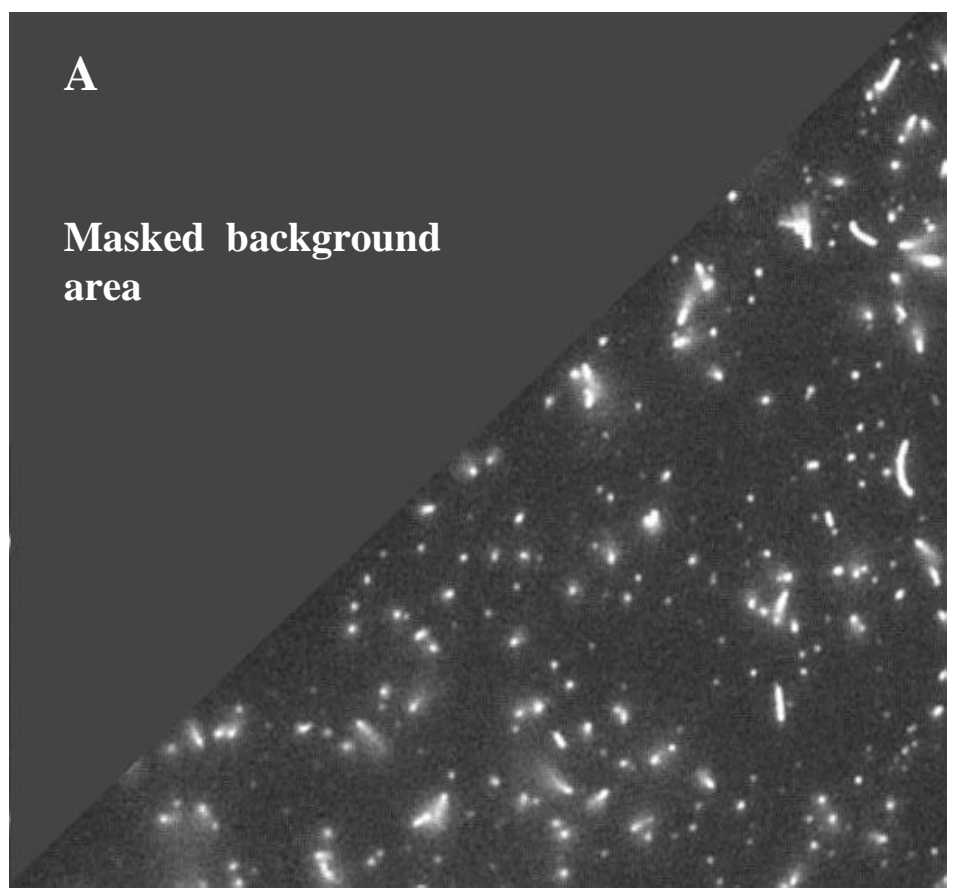



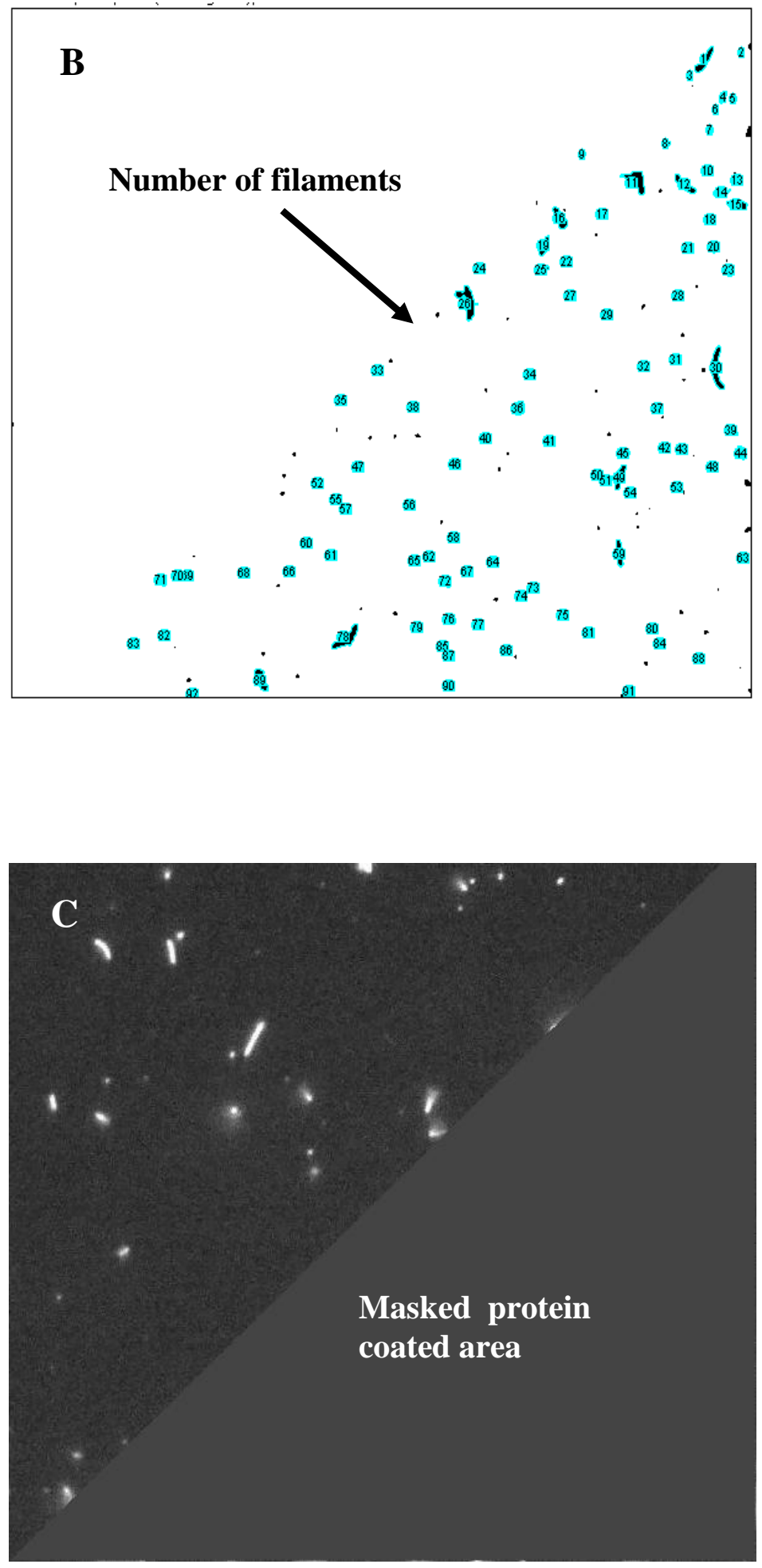


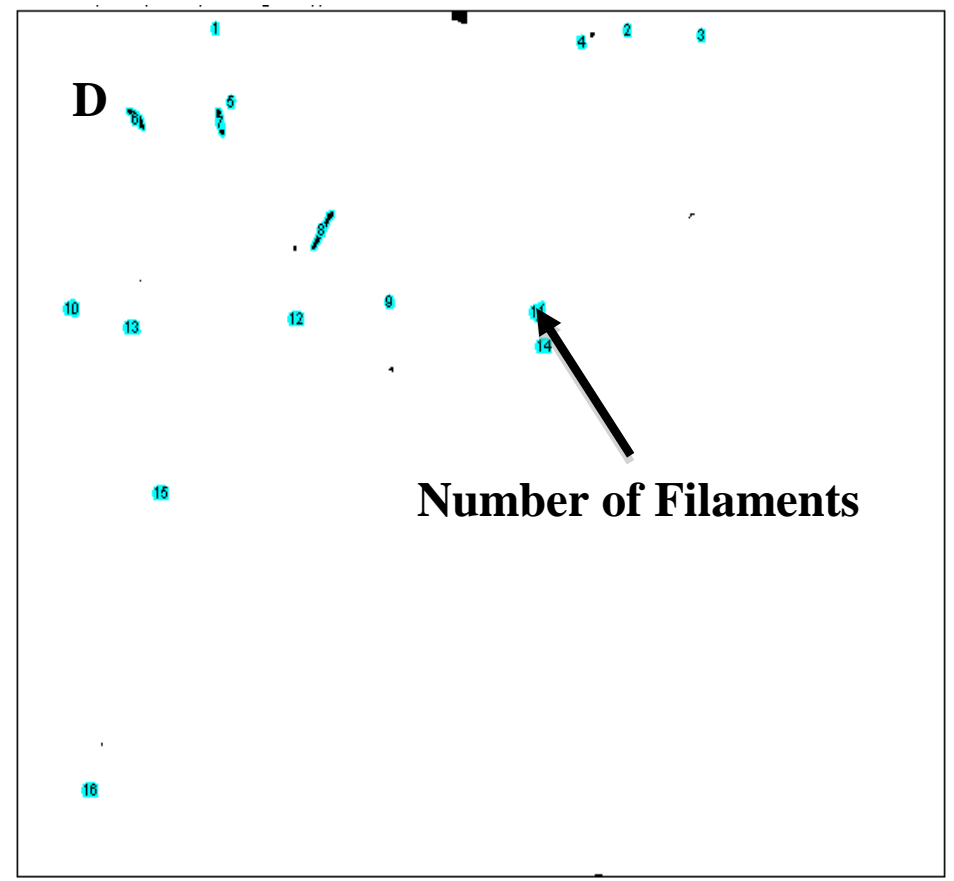

Figure 5.15 Determination of actin filaments by direct counting A) image with masked background area B) filament count in protein coated area C) image with masked protein coated area D) filament count in background area. The window dimensions are $62.55 \times 52.57$ microns.

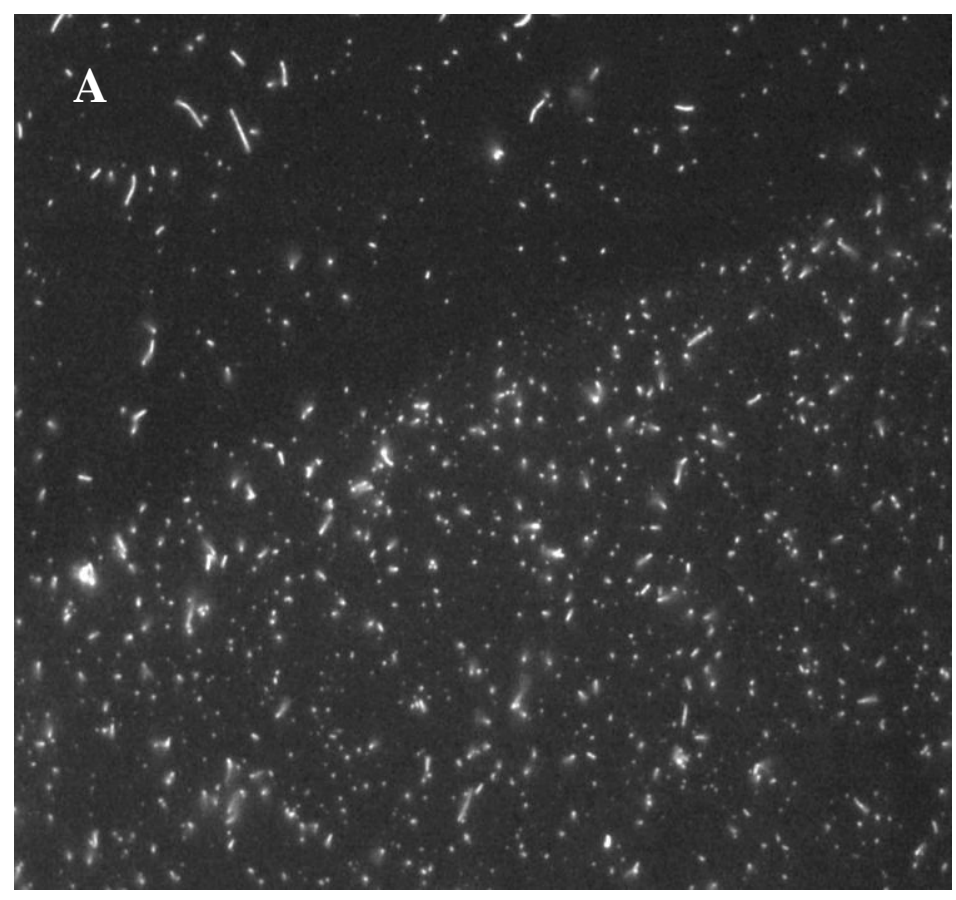



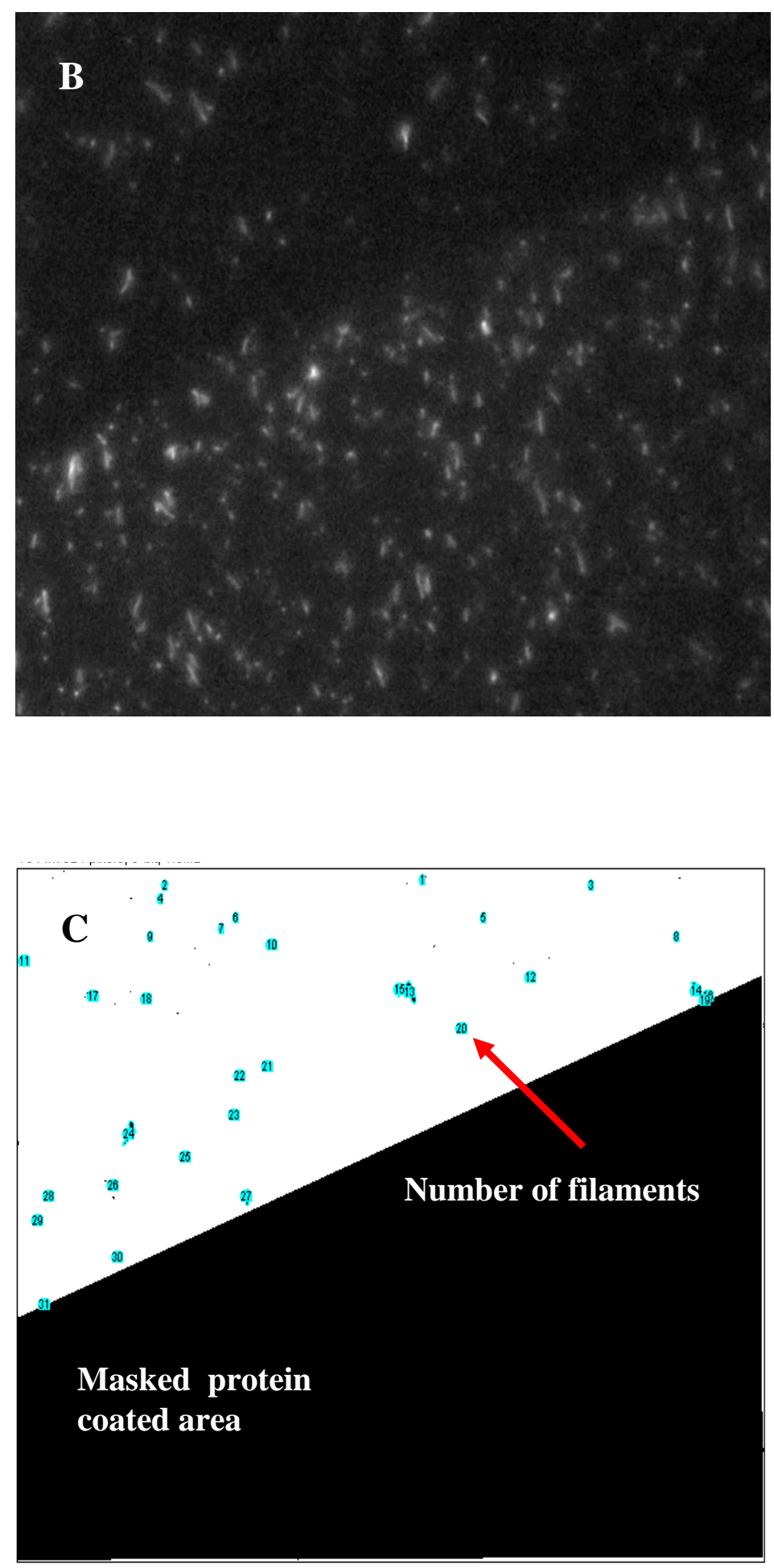


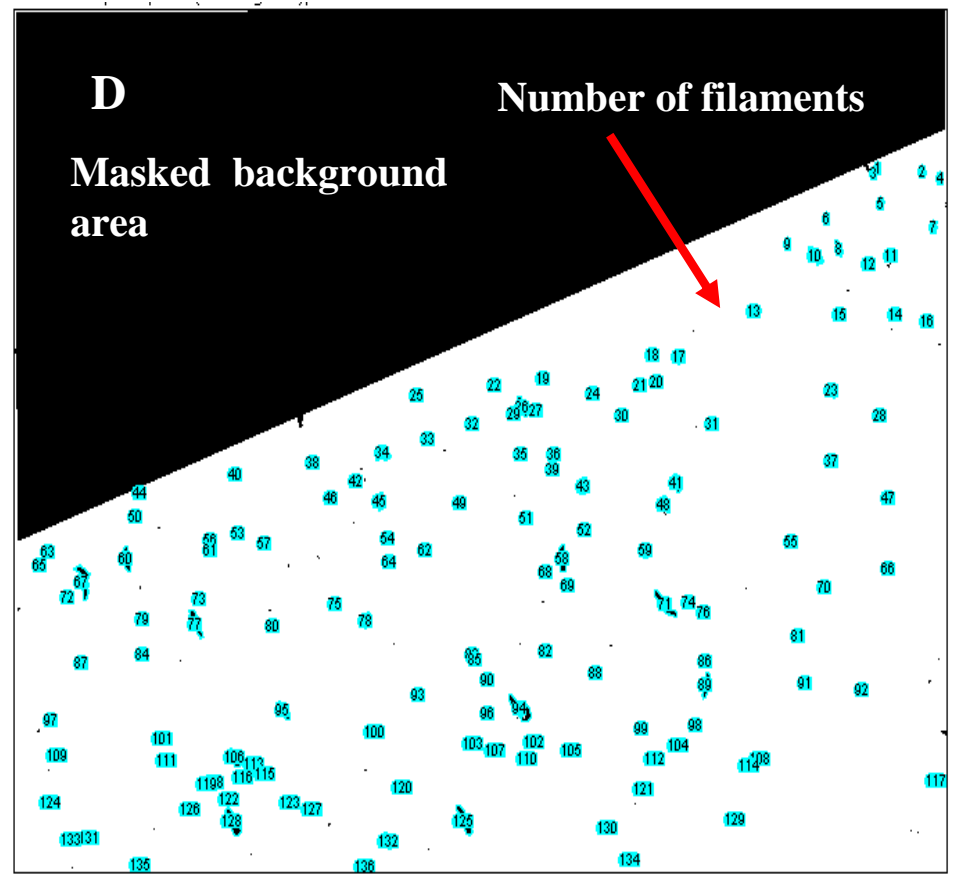

Figure 5.16 Determination of number of actin filaments after applying pressure driven flow. A) image before applying pressure driven flow B) image after applying pressure driven flow $C$ ) filament count in background area $D$ ) filament count in protein coated area. The window dimensions are $139.64 \times 106.39$ microns

Results showed a significant accumulation of filaments in areas covered with streptavidin, suggesting binding of the biotinylated gelsolin-capped actin on the streptavidin coated surface (Figure 5.18). 

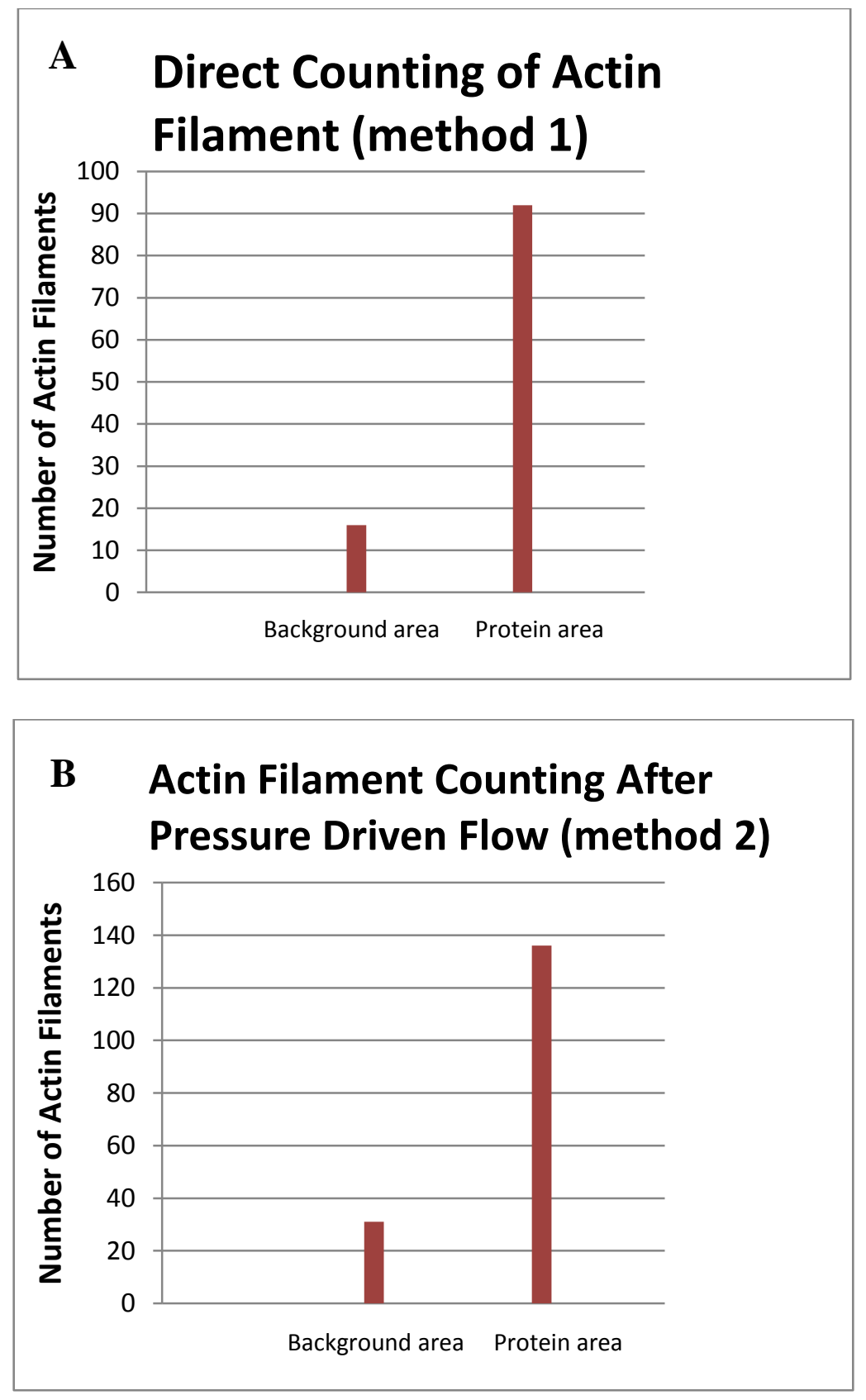

Figure 5.17. Characterization of bound biotinylated gelsolin-capped actin filaments A) direct counting B) counting after applying pressure driven flow 


\section{CHAPTER 6 CONCLUSIONS AND FUTURE DIRECTION}

The overall objective of this research project was to investigate the feasilibily of selective immobilization of actin motor proteins on streptavidin-coated surfaces with the aim of using actin for the development of more complex biomolecular systems. One of the most important studies accomplished in this thesis was to create a functional linkage between motor proteins and glass substrate. This aspect was accomplished by selectively patterning a biomolecular complex that served as a cross-linking agent between these motor proteins and the substrate.

This biomolecular complex consisted of a photo derivative of biotin and streptavidin protein, which provided an efficient method to link between an aminofunctionalized surface and actin filaments. Results presented in this thesis suggested that selective patterning of streptavidin protein onto amino-functionalized glass surfaces can be achieved via this linkage. Characterization of this system was accomplished with fluorescent microscopy. Patterning was achieved at a resolution of 10um. Although further research is warranted to achieve the goals of the overall project, the results presented here lay a foundation for future investigations.

To accomplish selective binding of biotinylated gelsolin-capped actin to the patterned surface was necessary to study the relationship between gelsolin and actin, as well as the characterization of non-specific binding of actin. Actin-gelsolin relationship studies revealed an inverse relationship between gelsolin concentration and actin filament length, which was expected since gelsolin not only acts as capping protein but also as a filament length control agent. Non specific binding studies indicated that BSA had the 
greatest blocking ability of the agents tested. Blocking is an essential component for achieving selective patterning of actin onto biotin-streptavidin coated surfaces. Further research will require effective blocking agents in order to achieve higher resolution. One specific blocking agent used in biological assays is nonfat dry milk. Nonfat dry milk contains many proteins which can effectively block nonspecific protein binding. Effective blocking of nonspecific actin binding is essential for the selectivity of actin immobilization. The efficacy of blocking of nonspecific binding is a key determiner of the resolution of patterning that can be achieved.

Finally, characterization results indicate a significant accumulation of actin filaments on streptavidin-coated areas suggesting that biotinylated gelsolin capped filaments bind to streptavidin coated surfaces due to bioaffinity interaction. However, further characterization of this system is needed for future applications. Specifically, AFM charcterization must be conducted on the surface as each component of the system is added. Experiments conducted as part of this thesis included AFM characterization of clean glass slides, APTES-functionalized glass slides, and APTES-functionalized glass slides with photobiotin pattern. A more complete understanding of the system will be achieved through further AFM characterization including: APTES-biotin-streptavidin coated surfaces, APTES-biotin-streptavidin-biotin coated surfaces, APTES-biotinstreptavidin-biotin-gelsolin coated surfaces, and APTES-biotin-streptavidin-biotingelsolin-actin coated surfaces. The use of patterning with and without blocking of nonspecific binding will also contribute to the characterization of the system. One limitation of AFM characterization on the Asylum MFP 3D, however, is the area that can be scanned, which is $90 \mathrm{um} \times 90 \mathrm{um}$. Due to this limitation and in order to measure surface 
roughness of multiple pattern features, patterns of high resolution must be used (patterns of 10um or less would provide multiple features within the AFM scan area). In addition, experiments coupling AFM with fluorescent microscopy will offer additional characterization that can be guided by fluorescent visualization in streptavidin and actincoated surfaces since these molecules fluoresce.

Additional tools for characterization may also prove useful. X-ray photoelectron microscopy can measure chemical composition of a sample and may be used to confirm the presence of amine groups on APTES-coated surfaces. Contact angle measurements could also be used to confirm the presence and uniformity of an APTES film. For characterization of actin filaments immobilization, scanning electron microscopy (SEM) and transmission electron microscopy (TEM) can be used. These tools allow visualization of actin filaments and may also be used to determine filament orientation. Actin filament orientation is important for the further development of the system, which will involve creation of unidirectional tracks of actin filaments that must be oriented parallel to the surface. Density of actin filaments can be visualized with SEM and TEM as well; filament density is another important feature in the future development of this system. Ultimately, characterization of biomolecular motor systems will lead to greater understanding of how these molecules interact and how they can be manipulated to develop useful nanoscale systems. 


\section{REFERENCES}

[1] http://www.zyvex.com/nanotech/feynman.html

[2] T. Jamison, Emergence of Nanotechnology, CSCE, September 2005.

[3] S. Iyer, B. Romanowicz and M. Laudon, "Biomolecular Motors", ConferenceProceedings, http://www.nsti.org/procs/Nanotech2004v1Nanotech 2004, Boston,MA, 2004, http://www.nsti.org/outreach/Biomolecular_Motors.

[4] P. Famouri, H. Takatsuki, L Hornak, K. Brown, R. Chilakamarri, A. Timperman, J. Lenke, P. Garnett and K. Kohama, "Nanofilament directional control within a hybrid microelectronic actin-myosin motility assay via integrated electrical addressing," NSTINanotech 2005, Anaheim, CA, pp. 663-639, May 2005.

[5] S. Wang, H. Arellano-Santoyo, P.A. Combs, and J.W. Shaevitz, "Actin-like cytoskeleton filaments contribute to cell mechanics in bacteria" PNAS, vol.107,pp. 91829185, 2010.

[6] K. Saladin. "Microscopic anatomy of skeletal muscle," Anatomy and Physiology Ed, M. Watnick, New York, NY: McGraw-Hill, pp. 409-422, 2004

[7] Georgios Tsiavaliaris, Setsuko Fujita-Becker and Dietmar J. Manstein, "Molecular engineering of a backwards-moving myosin motor", Nature, vol. 427, pp. 558-561, 2004.

[8] R. Stracke, K.J. Bohm ,J. Burgold, H.J Schacht and E. Unger, "Physical and technical parameters determining the functioning of a kinesin-based cell-free motor system", Nanotechnology, vol. 11, pp. 52-56, 2000.

[9] K.J. Bohm, R. Stracke, P. Mühlig and E. Unger, "Motor protein-driven unidirectional transport of micrometer-sized cargoes across isopolar microtubule arrays", Nanotechnology, vol. 12, pp. 238-244, 2001.

[10] R. Yokokawa, S. Takeuchi, T. Kon, M. Nishiura, K. Sutoh and H. Fujita, "Unidirectional Transport of Kinesin-Coated Beads on Microtubules Oriented in a Microfluidic Device”, Nano Letters, vol. 4, pp. 2265-2270, 2004.

[11] T. Ikawa, F. Hoshino, O. Watanabe, Y. Li , P. Pincus and C. R. Safinya, "Molecular Scale Imaging of F-Actin Assemblies Immobilized on a Photopolymer Surface", Physical Review Letters, vol. 98, pp. 018101-1 -018101-4, 2007.

[12] E.P. Diamandis and T.K. Christopoulos, "The Biotin-(Strept)Avidin System: Principles and Applicationsin Biotechnology", Clin. Chem, vol. 37, pp. 625-636,1991. 
[13] M. Gonzalez, L.A. Bagatolli, I. Echabe, J.L.R. Arrondo, C.E. Argaraña, C.R. Cantor, and G.D. Fidelio." Interaction of Biotin with Streptavidin", The Journal of Biological Chemistry, vol. 272, pp. 11288-11294, 1997

[14] F. Rusmini, Z. Zhong and J.Feijen, "Protein Immobilzation Strategies", Biomacromolecules, vol. 272 , pp. 1175-1189, 2007.

[15] D. V. Nicolau Jr and D. V. Nicolau," Towards a Theory of Protein Adsorption:Predicting the Adsorption of Proteins on Surfaces Using a Piecewise Linear Model Validated Using the Biomolecular Adsorption Database", 2nd Asia-Pacific Bioinformatics Conference (APBC2004)

[16] M.Malmsten." Formation of Adsorbed Protein Layers", Journal of Colloid and Interface Science, vol. 207, pp. 186-199, 1998.

[17] J-Y. Yoon, H-Y. Park, J-H. Kim and W-S. Kim, “Adsorption of BSA on highly carboxylated microspheres - quantitative effects of surface functional groups and interaction forces," J. Coll. Interface Sci. vol.177, pp. 613-620,1996.

[18] H. Ayhan, "Model protein BSA adsorption and covalent coupling onto methyl methacrylate based latex particles with different surface properties," J. Bioactive Compatible Poly. vol.17, pp. 271-283 2002.

[19] L.S. Wong, F. Khan and J. Micklefield, "Selective Covalent Immobilization: Strategies and Applications", Chem Rev, vol.109, pp. 4025-4053, 2009.

[20] J. Guisan "Immobilization of Enzymes and Cells," Methods in Biotechnology, Humana Press, 2006, pp. 107-108.

[21] A. Bernard, J. P. Renault, B. Michel, H. R. Bosshard and E. Delamarche, "Microcontact Printing of Proteins", Advanced Materials, vol. 12, pp 1067-1070, July 2000.

[22] Y. Xia and G. M. Whitesides,"Soft Liyhography",Annu. Rev. Mater. Sci., vol. 28, pp. 153-184, 1998.

[23] S.J. Clarson, J.A.Semlyen, Siloxane Polymers. Englewood Cliffs, NJ:Prentice Hall, pp. 1-2, 2000.

[24] J. Kuncová-Kallio and P.J. Kallio,” PDMS and its Suitability for Analytical Microfluidic Devices", Proceedings of the 28th IEEE EMBS Annual International Conference, 2006.

[25] Dow Corning Corporation, Sylgard ${ }^{\circledR}$ 184, material properties [Online]. Available: http://www2.dowcorning.com/DataFiles/090007c88020bcca.pdf. 
[26] J. L. Tan, J. Tien, and C.S. Chen," Microcontact Printing of Proteins on Mixed SelfAssembled Monolayers", Langmuir, 2002, vol. 18, pp. 519-523, 2002.

[27]. M. Schena, Protein Microarrays. Jones and Bartlett publishers, Missisagua,ON, pp2005.

[28] D.C. Trimbach, H. Stapert, J. van Orselen, K. D. Jandt, C. W. M. Bastiaansen and Dirk J. Broer, "Improved Microcontact Printing of Proteins using HydrophilicThermoplastic Elastomers as Stamp Materials", Advanced Engineering Materials, vol.9, pp.1123-1128, 2007.

[29] R. D. Piner, J. Zhu, F. Xu, S. Hong and C. A. Mirkin," "Dip-Pen" Nanolithography”, Science, vol. 283, pp 661-663, 1999.

[30] J. H. Lim, D.S. Ginger, K.B. Lee, J. Heo, J.M. Nam and C.A. Mirkin CA, "Directwrite dip-pen nanolithography of proteins on modified silicon oxide surfaces", Angewandte Chemie International Edition, vol. 42, pp.2309-2312, 2003.

[31] M.L. Harris , A. Doraiswamy , R.J. Narayan , T.M. Patz and D.B. Chrisey,"Recent progress in CAD/CAM laser direct-writing of biomaterials", Materials Science and Engineering C, vol. 28 , pp. 359-365, 2008.

[32] C.Z. Dinu , V. Dinca, J. Howard, and D.B. Chrisey, "Printing technologies for fabrication of bioactive and regular microarrays of streptavidin", Applied Surface Science, vol. 253, pp. 8119-8124, 2007.

[33] M. Mrksich, L.E. Dike, J. Tien, D.E. Ingber and G.M. Whitesides, "Using Microcontact Printing to Pattern the Attachment of Mammalian Cells to Self-Assembled Monolayers of Alkanethiolates on Transparent Films of Gold and Silver", Experimental Cell Research, vol. 235, pp. 305-313, 1997.

[34] L.M. Lee, R.L. Heimark, J.C. Baygents and Y. Zohar, "Self-Aligned Immobilization of Proteins Utilizing PEG Patterns", Nanotechnology, vol.17, pp. S29-S33, 2006.

[35] G. Blagoi, S. Keller, F. Persson, A. Boisen and M. H. Jakobsen, "Photochemical Modification and Patterning of SU-8 Using Anthraquinone Photolinkers," Langmuir , vol 24 , pp 9929-9932, 2008.

[36] D.V. Nicolau, T. Taguchi, H. Taniguchi and S. Yoshikawa, "Micron-Sized Protein Patterning on Diazonaphthoquinone/Novolak Thin Polymeric Films," Langmuir, vol 14, pp 1927-1936, 1998.

[37] M. Hengsakul and A. E. G. Cass, "Protein Patterning with a Photoactivatable Derivative of Biotin," Bioconjugate Chem., 1996, vol 7, pp 249-254, 1996. 
[38] M. Qin, S. Hou, L. Wang, X.Feng, R. Wang, Y. Yang, C Wang, L. Yu, B. Shao and M. Qiao, "Two Methods for Glass Surface Modification and their Application in Protein Immobilization", Colloids and Surfaces B: Biointerfaces, vol 60, pp. 243-249, 2007.

[39] R.N. Orth, T.G. Clark and H.G. Craighead, "Avidin-Biotin Micropatterning Methods for Biosensor Applications”, Biomedical Microdevices, vol 5, pp.29-34, 2003.

[40] C.C. Meek and P. Pantano,"Spatial Confinement of Avidin Domains in Microwell Arrays", Lab on a chip, vol 1, pp 158-163, 2001.

[41] H. J. Choia, N. H. Kimb, Bong Hyun Chunga, and G. H. Seong, "Micropatterning of biomolecules on glass surfaces modified with various functional groups using photoactivatable biotin," Analytical Biochemistry, vol 347, pp 60-66, December 2005.

[42] W. Kabsch and J. Vandekerckhove, "Structure and Function of Actin", Anna. Rev. Biophys. Biomol. Struct, vol. 21, pp. 49-76, 1992.

[43] T. Pollard and J.A. Cooper, "Actin and Actin-Binding Proteins. A Critical Evaluation of Mechanisms and Functions", Ann. Rev. Biochem, vol. 55, pp. 987-1035, 1986.

[44] M. Stoeckelhuber, A.A. Noegel, C. Eckerskorn, J. Köhler, D. Rieger and M. Schleicher, "Structure/function studies on the pH-dependent actin-binding protein hisactophilin in Dictyostelium mutants", Journal of Cell Science, vol. 109, pp.1825-1835, 1996.

[45] K.M. Flaherty, D.B. McKay, W. Kabsch and K.C. Holmes, "Similarity of the threedimensional structures of actin and the ATPase fragment of a 70-kDa heat shock cognate protein", Proc. Nati. Acad. Sci. USA, vol. 88, pp. 5041-5045, 1991.

[46] K.R. Ayscough and D.G. Drubin, "ACTIN: General Principles from Studies in Yeast”, Annu. Rev. Cell Dev. Biol, vol.12, pp. 129-160, 1996.

[47] B. Alberts, A. Johnson, J. Lewis, M. Raff, K. Roberts and P. Walter, Molecular Biology of the Cell, 3rd edition, New York: Garland Science; 1994, http://www.ncbi.nlm.nih.gov/bookshelf/br.fcgi?book=cell\&part=A4274

[48] Actin Assembles to Form Microfilaments. http://www.wiley.com/college/pratt/0471393878/student/animations/actin_myosin/actin_ $\underline{\text { myosin.swf }}$

[49] Thermo Scientific Avidin-Biotin Technical Handbook www.piercenet.com/files/1601675_AvBi_HB_INTL.pdf

[50] Wikipedia, http://en.wikipedia.org/wiki/Biotin 
[51] G. T. Hermanson. Bioconjugate Techniques, Academic Press, second edition, San Diego pp. 900-901, 2008.

[52] S. Freitag, I. L. Trong, L. Klumb, P. S. Stayton, and R. E. Stenkamp, "Structural studies of the streptavidin binding loop," Protein Science, vol. 6, pp. 1157-1166, 1997.

[53] P. C. Weber, D. H. Ohlendorf, J. J. Wendoloski, and F. R. Salemme, "Structural Origins of High-Affinity Biotin Binding to Streptavidin," Science, vol. 243, pp. 86-88, 1989.

[54] http://www.chemie.unibas.ch/ ward/research.html

[55] A. Holmberg, A. Blomstergren, O. Nord, M. Lukacs, J. Lundeberg and M. Uhlén, "The biotin-streptavidin interaction can be reversibly broken using water at elevated temperatures", Electrophoresis, vol. 26, pp. 501-510, 2005.

[56] Q. Li, S. Gusarov, and A. Kovalenko, “ Molecular Dynamics Study of Streptavidin Binding to Surface-Immobilized Biotin", International Symposium on Computer Science and Computational Technology, pp.767-770, 2008.

[57] H. Grubmüller, B. Heymann and P. Tavan, "Ligand Binding: Molecular Mechanics Calculation of the Streptavidin-Biotin Rupture Force", Science, vol. 271, pp. 997 - 999, 1996

[58] S. Izrailev, S. Stepaniants, M. Balsera, Y. Oono, and K. Schulten,” Molecular Dynamics Study of Unbinding of the Avidin-Biotin Complex", Biophysical Journal, vol. 72, pp.1568-1581, 1997.

[59] H.Q Sun, M. Yamamoto, M. Mejillano and H. L. Yin, "Gelsolin, a Multifunctional Actin Regulatory Protein”, Journal of Biological Chemistry, vol. 274, pp.33179-33182, 1999.

[60] P.J. McLaughlin, J.T. Gooch, H.G. Mannherz and A.G. Weeds, "Structure of gelsolin segment 1-actin complex and the mechanism of filament severing", Nature, vol. 364, pp. $685-692,1993$.

[61] H. L. Yin, J. H. Hartwig, K. Maruyama and T. Stossel, "Ca2+ control of actin filament length. Effects of macrophage gelsolin on actin polymerization. J Biol Chem., vol. 256, pp. 9693-9697, 1981.

[62] David J Kwiatkowski, "Functions of gelsolin: motility, signaling, apoptosis, cancer", Current Opinion in Cell Biology, vol. 11, pp. 103-108, 1999.

[63] S. A. Brooks, W. P. Ambrose and W.G. Kuhr, "Micrometer Dimension Derivation of Biosensor Surfaces Using Confocal Dynamic Patterning". Anal. Chem., vol. 71, pp. 2558-2563, 1999. 
[64] J. A. Howarter and J. P. Youngblood, "Optimization of Silica Silanization by 3Aminopropyltriethoxysilane”, Langmuir , vol 22, pp, 11142-11147, 2006.

[65] P. Heiney, K. Gruneberg, J. Fang, C. Dulcey and R. Shashidhar "Structure and growth of chromophore-functionalized (3-aminopropyl)triethoxysilane self-assembled on silicon”, Langmuir, vol. 16, pp. 2651-2657, 2000.

[66] H.J. Kang and F. D. Blum, "Structure and Dynamics of Amino Functional Silanes Adsorbed on Silica Surfaces”, J. Phys. Chem. vol. 95, pp. 9391-9396, 1991.

[67] M. Etienne and A. Walcarius, "Analytical investigation of the chemical reactivity and stability of aminopropyl-grafted silica in aqueous medium", talanta, vol. 59, pp. 1173-1188, 2003.

[68] Sigma Aldrich A8806 "Albumin from bovine serum", http://www.sigmaaldrich.com/catalog/ProductDetail.do?D7=0\&N5=SEARCH_CONCA T_PNO|BRAND_KEY\&N4=A8806|SIGMA\&N25=0\&QS=ON\&F=SPEC.

[69] Thermo Scientific "MS-(PEG) $)_{\mathrm{n}} \quad$ PEGylation reagents", http://www.piercenet.com/products/browse.cfm?fldID=20CD10B3-1F10-4A87-B76597DAB2FB6681.

[70] J.Y. Yoon, J. H. Lee, J.H. Kim and W.S. Kim, "Separation of serum proteins with uncoupled microsphere particles in a stirred cell", Colloids and Surfaces B: Biointerfaces, vol. 10, pp. 365-377, 1998.

[71] Z.G. Peng, K. Hidajat, and M.S. Uddin, "Adsorption of bovine serum albumin on nanosized magnetic particles", Journal of Colloid and Interface Science, vol. 271, pp. 277-283, 2004.

[72] J. M. Corbett, H. J. Why, C. H. Wheeler, P. J. Richardson, L. C. Archard, M. H. Yacoub and M. J. Dunn, "Cardiac protein abnormalities in dilated cardiomyopathy detected by two-dimensional polyacrylamide gel electrophoresis", Electrophoresis, vol. 19, pp. 2031-2042, 1998. 


\section{APPENDIX A}

\section{A.1 Characterization Tools}

This section presents characterization tools used during experiments. The two main instruments used were fluorescent microscopy to assess the presence of fluorescenttagged elements and atomic force microscopy for surface characterization of functionalized glass substrates.

\section{A.1.1 Fluorescent Inverted Microscope}

Due to the nature of these experiments, the single most important piece of equipment was the Epi-fluorescent inverted microscope (Nikon Eclipse TE200). This microscope was used to detect the presence of specimens (detection of fluorescent-tagged proteins), which are the source of light during detection. Fluorescent microscopy functions when a specimen or sample is shinned with light at a specific wavelength that matches the excitation wavelength of the sample. The atoms on fluorescent-tagged molecules become excited and jump to a higher energy state. While returning to a lower energy state, atoms generate light due to the emission of photons.

Epi-fluorescent microscopes get their name from the fact that light passes through the objective before it reaches the specimen. The excitation light source comes from a light bulb generally mercury or xenon (arc lamp source) that is placed on the lamp house (Figure A.1). 


\section{Detection system}

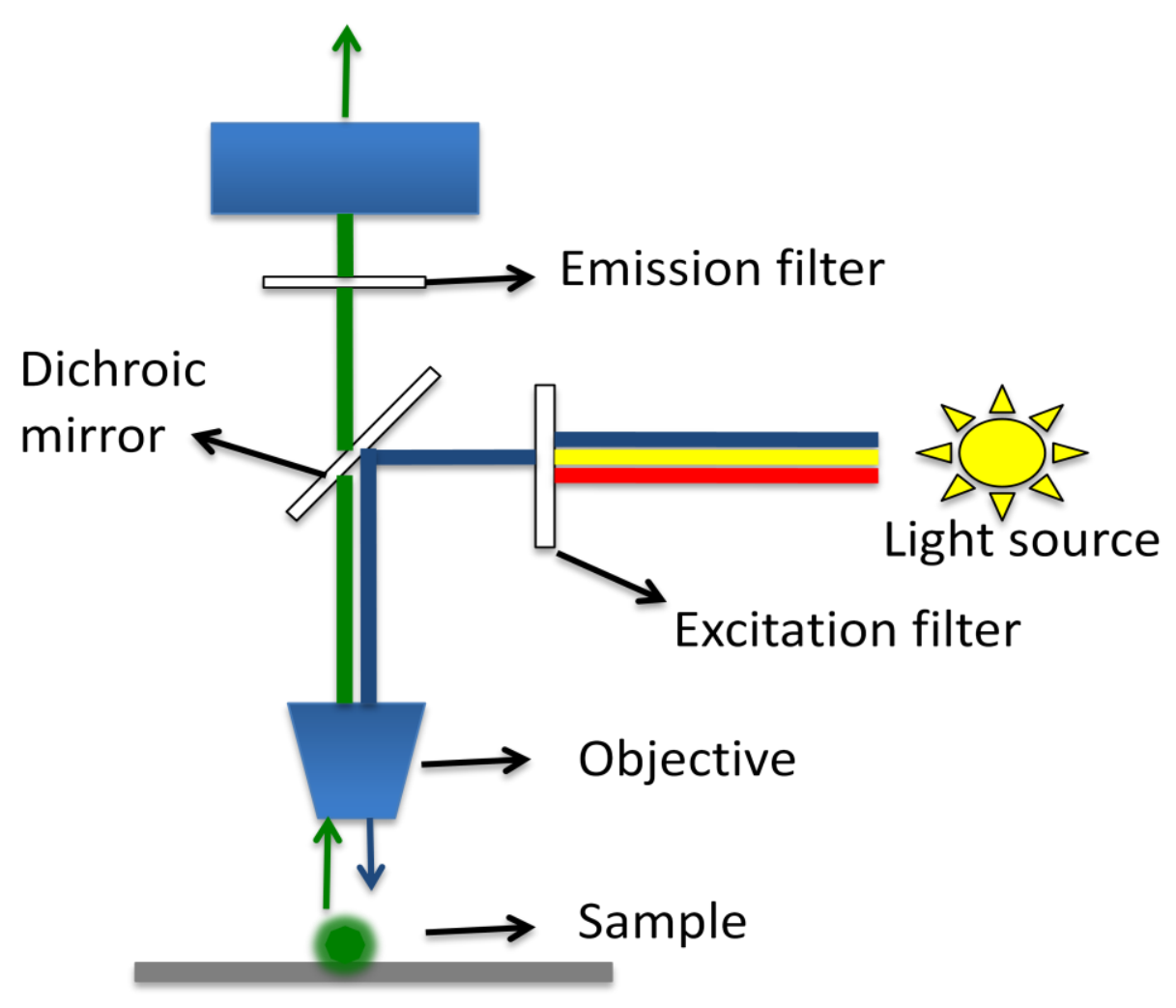

Figure A.1 Schematic of an epi-fluorescent inverted microscope.

Light from this source is multispectral and can be passed through a set of filters: excitation filter, dichromatic beamsplitter (known as dichroic) and an emission filter. The excitation filter separates the multispectral light into a wavelength specific light commonly green, blue and ultraviolet. Table A.1 shows the specifications of the microscope excitation filters used in the studies described here. 


\begin{tabular}{|c|c|c|c|}
\hline Filter Model & $\begin{array}{c}\text { Excitation } \\
\text { Wavelength } \\
(\mathbf{n m})\end{array}$ & $\begin{array}{c}\text { Dichromatic Mirror } \\
\text { Cut-on Wavelength } \\
(\mathbf{n m})\end{array}$ & $\begin{array}{c}\text { Emission } \\
\text { Wavelength } \\
(\mathbf{n m})\end{array}$ \\
\hline UV-2E/C & $340-380$ & 400 & $435-485$ \\
\hline B-2E/C & $465-495$ & 505 & $515-555$ \\
\hline G-2E/C & $528-553$ & 565 & $590-650$ \\
\hline
\end{tabular}

Table A.1 Filters specifications of Nikon Eclipse TE-200

The selected light from the excitation filter is reflected by the dichromatic beamsplitter, which only reflects light of short wavelength (larger wavelength light pass through the mirror). The light is then shined onto the sample where the fluorescent molecules in the sample, called fluorophores, are excited to a higher energy state, emitting photons upon relaxation. The period of time between adsorption and emission of light is on the order of microseconds. The emitted light goes through the dichroic mirror (emitted light has longer wavelength than excitation light therefore passes through the mirror) and is collected by the microscope objective and then passed through the emission filter that filters out any remaining light not coming from the sample.

The objective not only serves as collector, but also helps to correct the collected emitted light from the source, the refractive index of which depends on the type of 
objective and the media used between the sample and objective (most commonly air or oil). For example, in high magnification objectives, oil can be used as media between the sample and objective because it increases the numerical aperture of the objective due to the change of the refractive index allowing collection of more light from the sample.Table A.2 shows a list of objective used during fluorescent characterization of experiments

\begin{tabular}{|c|c|c|}
\hline Task & Type of Filter & Objective \\
\hline $\begin{array}{c}\text { Protein } \\
\text { patterning: }\end{array}$ & $\begin{array}{c}\text { B-2E/C for FITC } \\
\text { Streptavidin and DyLight } \\
\text { Streptavidin, } \\
\text { G-2E/C For TRITC } \\
\text { Streptavidin }\end{array}$ & $\begin{array}{l}\text { Magnification: } 4 \mathrm{x} \\
\text { Numerical Aperture: } \\
\text { Working distance: } 0.20 \mathrm{~mm} \\
\text { Optical properties: } \\
\text { Optical Correction: plan Flour } \\
\text { Coverglass thickness: } 1.2 \mathrm{~mm} \\
\text { Magnification: } 10 \mathrm{x} \\
\text { Numerical Aperture: } 0.25 \text { air } \\
\text { Working distance: } 1.2 \mathrm{~mm} \\
\text { Optical properties: } \\
\text { Optical Correction: none } \\
\text { Coverglass thickness: } 1.2 \mathrm{~mm} \\
\text { Magnification }=40 \mathrm{x} \\
\text { NA =1.35 oil } \\
\text { Working distance }=0.20 \mathrm{~mm} \\
\text { Optical properties=DIC H } \\
\text { Optical Correction= planFlour } \\
\text { Coverglass thickness: } 0.17 \mathrm{~mm} \\
\\
\text { Magnification: } 60 \mathrm{x} \\
\text { NA: } 1.35 \text { oil } \\
\text { Working distance: } 0.20 \mathrm{~mm} \\
\text { Optical properties: DIC H } \\
\text { Optical Correction: planFlour } \\
\text { Coverglass thickness :0.17mm } \\
\text { Magnification: } 100 \mathrm{x} \\
\text { NA:1.35 oil } \\
\text { Working distance: } 0.20 \mathrm{~mm} \\
\text { Optical properties: DIC H } \\
\text { Optical Correction: plan Flour } \\
\text { Coverglass thickness:0.17mm }\end{array}$ \\
\hline
\end{tabular}




\begin{tabular}{|c|c|c|}
\hline $\begin{array}{l}\text { Microcontact } \\
\text { Imprint }\end{array}$ & $\begin{array}{c}\text { B-2E/C for FITC } \\
\text { Streptavidin and DyLight } \\
\text { Streptavidin, } \\
\\
\text { G-2E/C For TRITC } \\
\text { Streptavidin }\end{array}$ & $\begin{array}{l}\text { Magnification: } 4 \mathrm{x} \\
\text { Numerical Aperture: } \\
\text { Working distance: } 0.20 \mathrm{~mm} \\
\text { Optical properties: } \\
\text { Optical Correction: planFlour } \\
\text { Coverglass thickness: } 1.2 \mathrm{~mm} \\
\text { Magnification: } 10 \mathrm{x} \\
\text { Numerical Aperture: } 0.25 \text { air } \\
\text { Working distance: } 1.2 \mathrm{~mm} \\
\text { Optical properties: } \\
\text { Optical Correction: none } \\
\text { Coverglass thickness: } 1.2 \mathrm{~mm}\end{array}$ \\
\hline $\begin{array}{c}\text { Actin Filaments } \\
\text { Observation }\end{array}$ & $\begin{array}{c}\text { G-2E/C Rhodamine } \\
\text { Phalloidin labeled Actin } \\
\text { Filaments }\end{array}$ & $\begin{array}{l}\text { Magnification: } 60 \mathrm{x} \\
\text { NA: } 1.35 \text { oil } \\
\text { Working distance: } 0.20 \mathrm{~mm} \\
\text { Optical properties: DIC H } \\
\text { Optical Correction: planFlour } \\
\text { Coverglass thickness :0.17mm } \\
\\
\text { Magnification: } 100 \mathrm{x} \\
\text { NA: } 1.35 \text { oil } \\
\text { Working distance: } 0.20 \mathrm{~mm} \\
\text { Optical properties: DIC H } \\
\text { Optical Correction: planFlour } \\
\text { Coverglass thickness: } 0.17 \mathrm{~mm}\end{array}$ \\
\hline
\end{tabular}

TableA.2 Type of Filters and Objectives used during experiments

\section{Atomic Force Microscopy (AFM)}

The development of new microscopy techniques, such as scanning probe microscopy (SPM), has allowed researchers to fully characterize surfaces at the atomic level. SPM has become useful in areas such physics, engineering, biology or chemistry and also has allowed significant progress in the field of nanotechnology. 
There are two types of imaging modes used in AFM imaging: contact mode and tapping mode (Figure A.2).

A

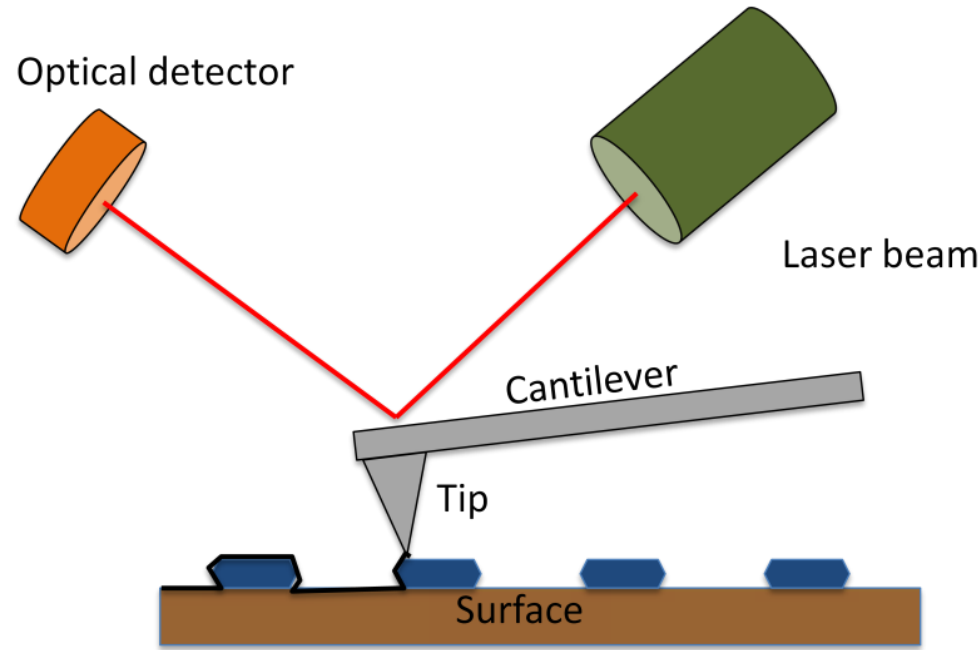

B

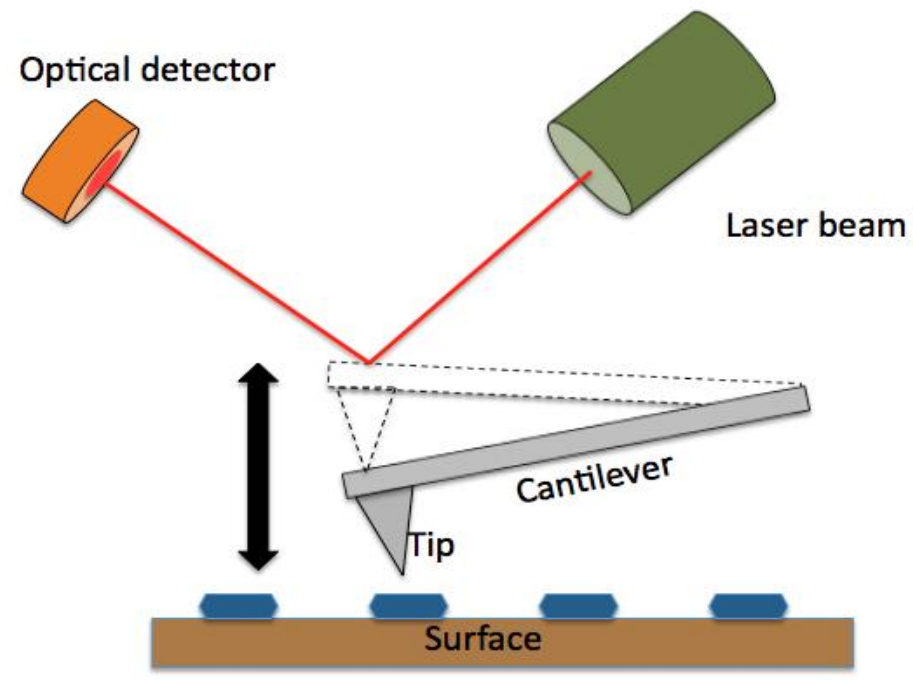

Figure A.2 Schematic of AFM imaging modalities. A)Contact mode B) tapping mode

Specific studies in this thesis utilized AFM imaging to characterize the deposited layer of APTES on glass substrates. The type of AFM system used was the Asylum 
Research MPF3D coupled to Igor pro 6.0.4 software to control the system and for image analysis. This AFM head is mounted on an isolation system, which functions to reduce noise that could affect the quality of the scanned images (Figure A.3).

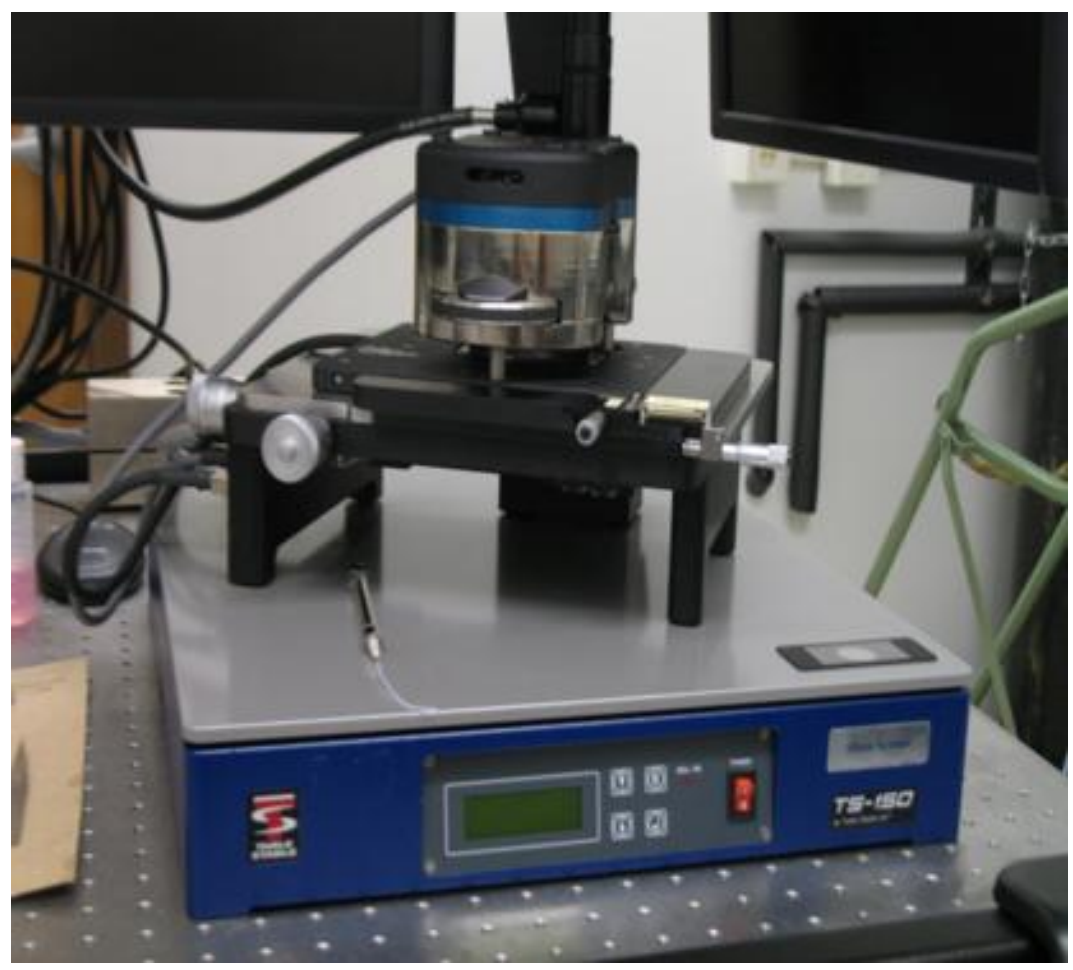

Figure A.3 MFP3D Asylum Research AFM on top of isolation system

\section{A.2 Digital Data Recording and Analysis}

This section presents the instruments and software used to acquire, record and process the fluorescent images and videos obtained from the fluorescent microscope and AFM systems. 


\section{A.2.1 CCD Camera and Computer Interface}

The fluorescent microscope was used to observe fluorescent specimens during experimentation (Figure A.4). For image acquisition, an Orca-EG digital camera model C4742-80-12AG Hamamatsu Photonics) was used. This camera is interfaced with the computer (PC Windows XP operating system) via IEEE 1894 protocol. The camera is capable to acquire images at a rate of 8.9 frames per seconds in a 1344 x1024 window.
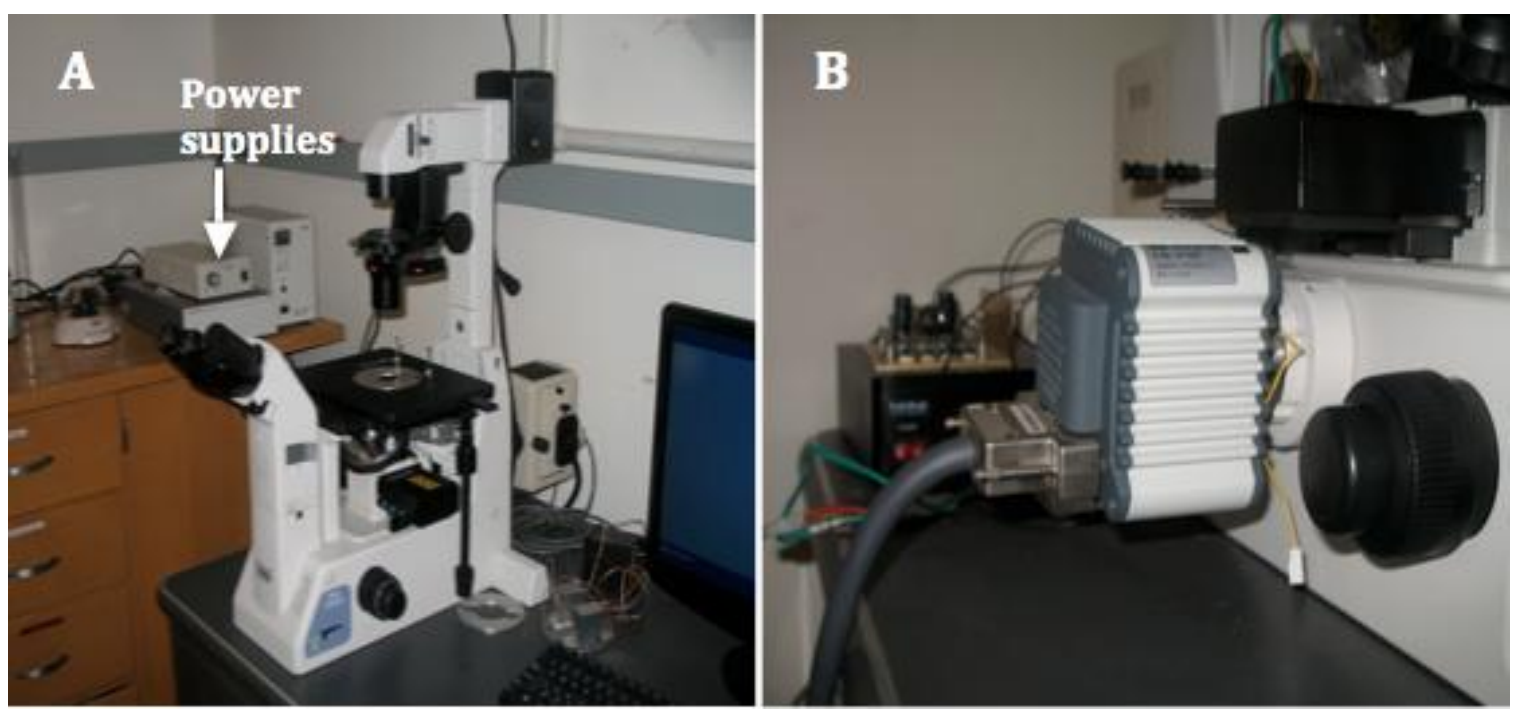

Figure A.4 Complete microscope system A) Fluorescent inverted microscope including the power supplies B) Orca digital camera

\section{A.2.2 Image Recording and Processing}

The acquired images and movies were recorded and processed by a package program that came with the microscope and CCD camera. "Wasabi" software allowed recording of images and movies and also allowed the computer command of the CCD camera parameters such as exposure time, windows size, and frame rate through the computer interface (Figure A.5). Also, the program allows users to perform typical image 
processing routines such as control of image contrast, tint images (when working with two different fluorescent molecules) as well as intensity calculation, among other operations.

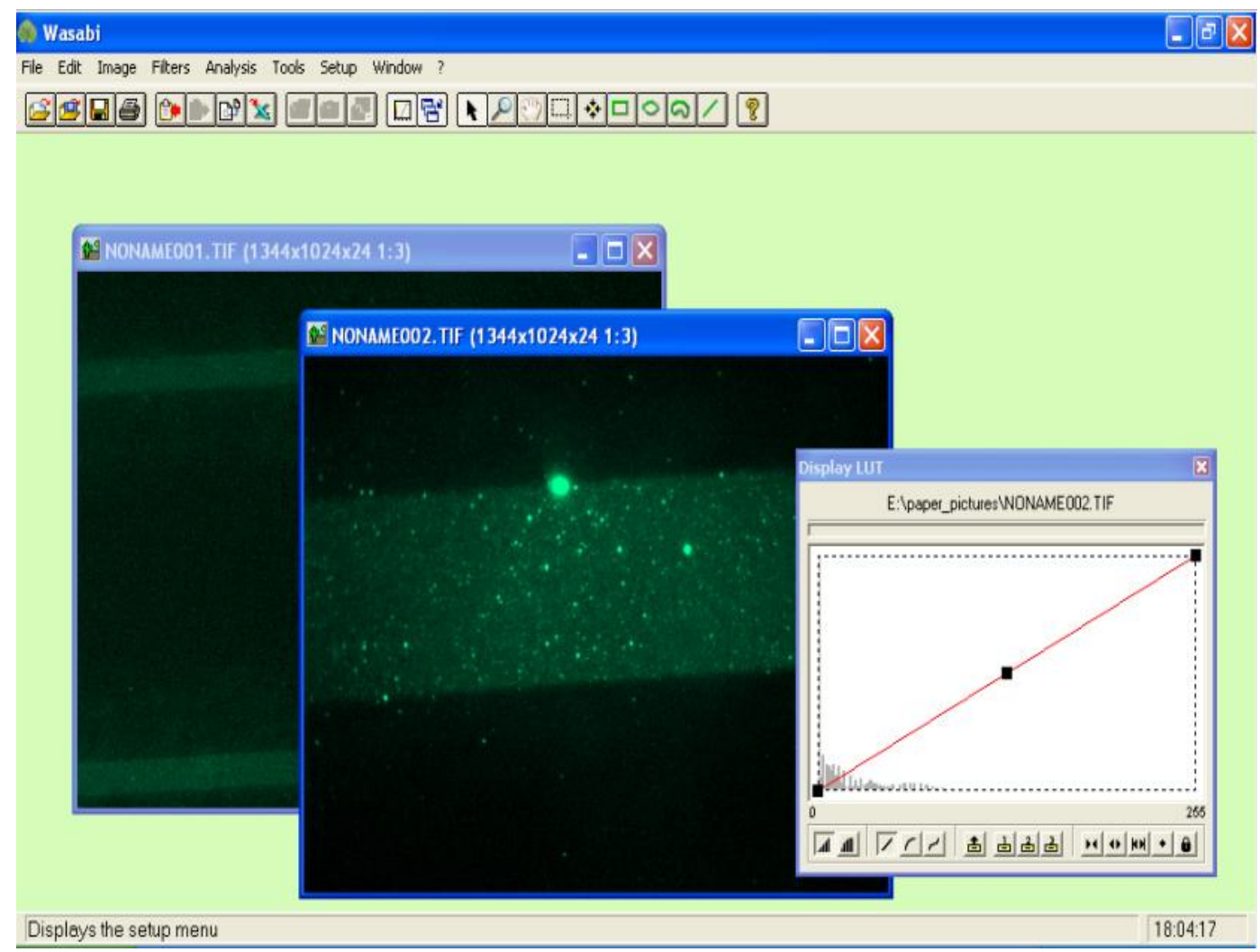

Figure A.5 Graphical user interface of Wasabi

This software can record and create AVI movies, but during the movie creation the frame rate is altered to 15 frames per second therefore a post processing video editor is required. This post processing can be done by using open source video processing software such as VirtualMod or Virtualdub, which can adjusts the frame per second rate to the original frame rate the movie had when recorded. Figure A.6 shows the graphical interface of the VirtualDub software used for video editing during experiments. 


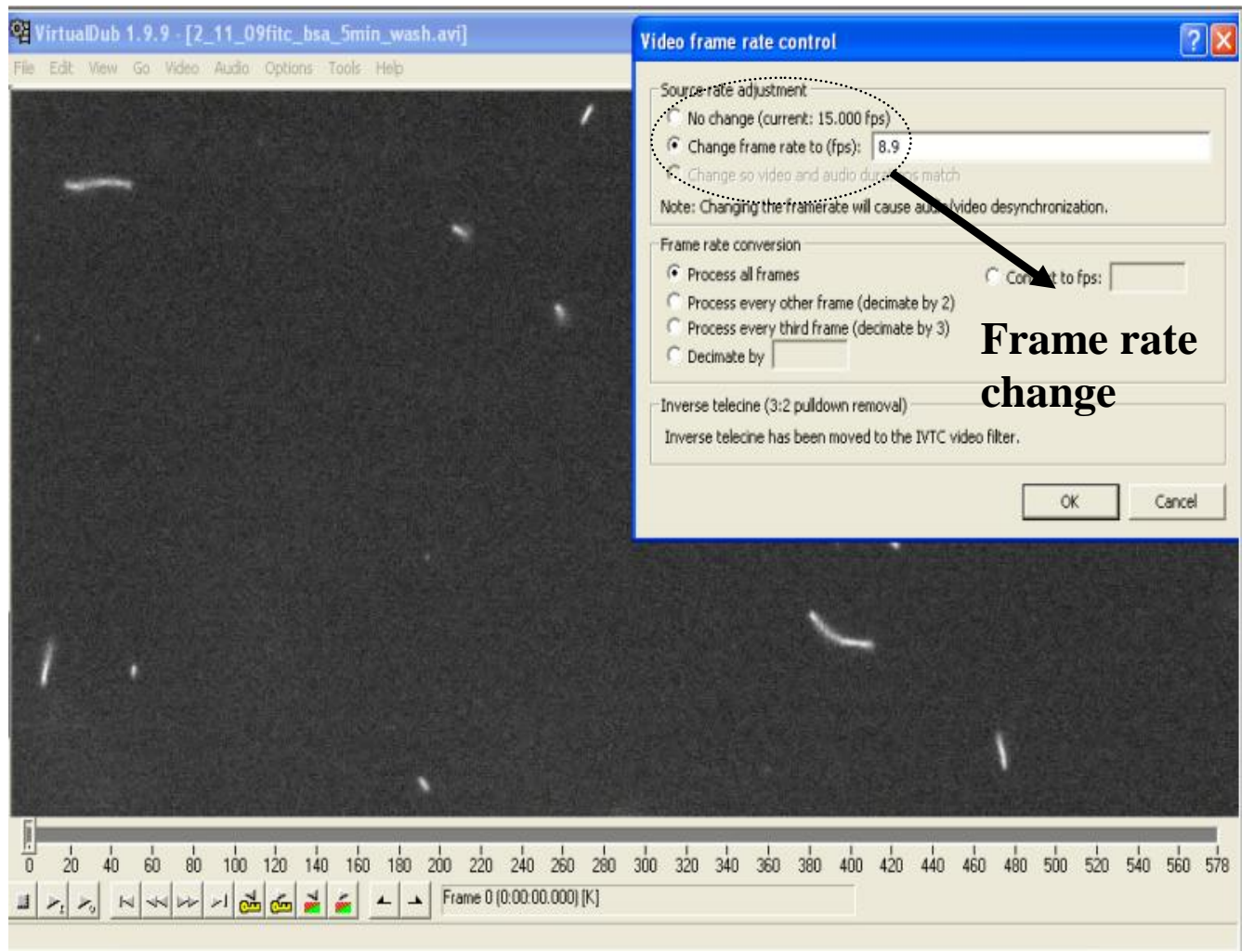

Figure A.6 Graphical user interface of VirtualDub video editor

For further analysis of the images obtained from fluorescent microscopy, an open source image processing software (Image J) was used. This powerful software allowed performing diverse operations on images such as counting of elements, algebraic operations such as addition or subtraction and determination of elements length and area as well as scaling of the images from pixels to units of length (mainly micrometers).

For the analysis and image processing of the AFM images, Igor pro 6.0.4 was used. This program not only allowed control of the AFM system parameters such as gain, set point voltage (amount of force applied to the tip), image modality, scan size, scan speed but was also capable of performing post processing of the image and calculation of the parameters such as surface roughness (Figure A.7). 


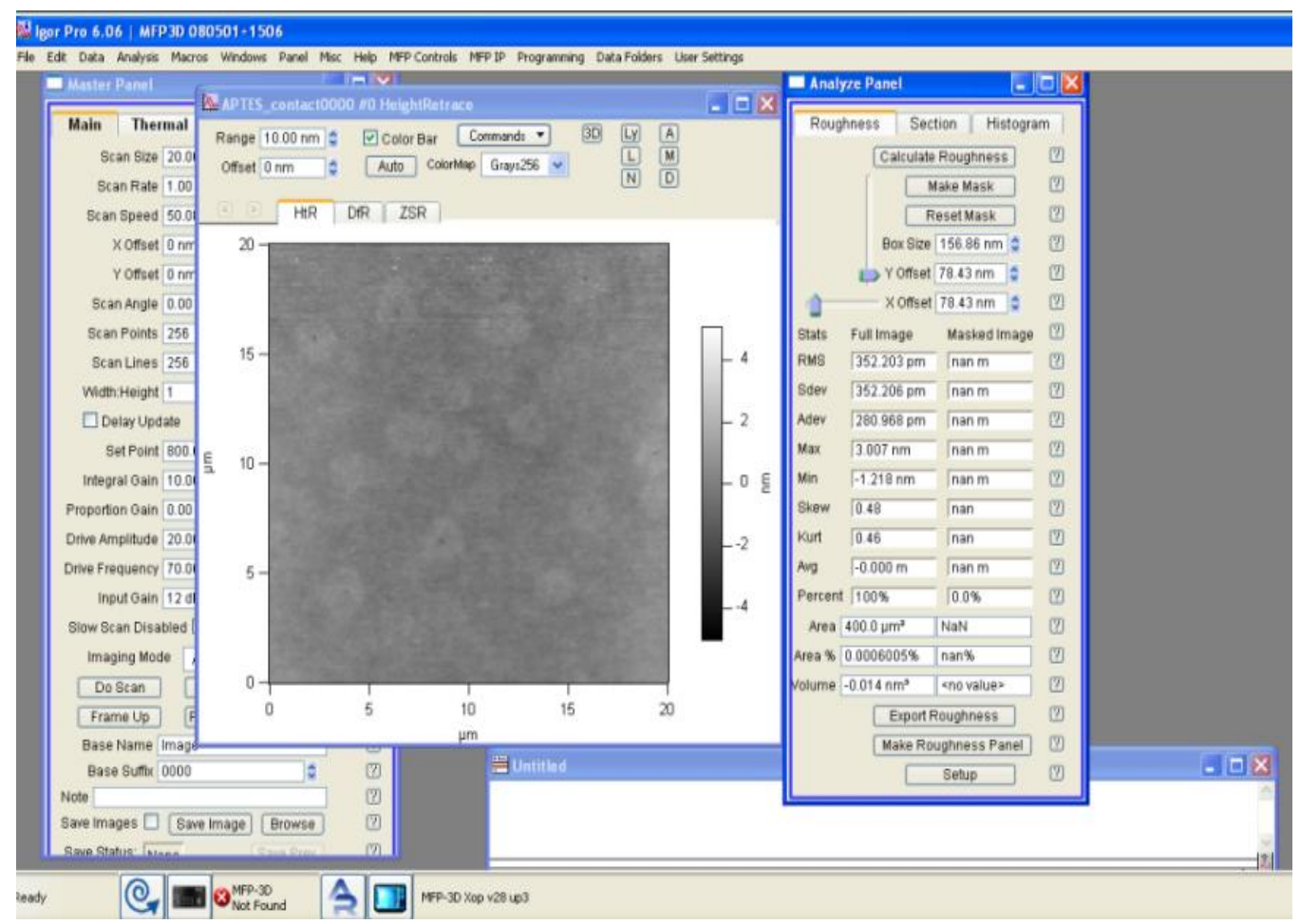

Figure A.7 Graphical user interface of Igor Pro 6.0.4, showing the analysis panel

\section{A.3 Microfabrication Instruments}

Most of the protein patterning experiments were conducted in the WVU nano clean room facility. The cleanroom is a 3300 square feet facility divided into three classes of areas: class 100, and two class 10,000. These names correspond to a classification according to the maximum number of particles per cubit feet allowed in the room. This facility is used mostly for microfabrication due to the specific conditions required to fabricate miniature devices.

Diverse microfabrication processes are executed there, such photolithograpy, etching, metallization or material growth. These processes form the basis of microdevice fabrication. The cleanroom areas were appropriate to work since the environment where 
biomolecules were exposed was significantly cleaner and because all instruments needed for the process were located there. An additional advantage was the ability to manipulate photosensitive materials, such as the photobiotin, in the cleanroom because illumination in some areas is by yellow light, which is ideal for working with photosensitive material.

\section{A.3.1 Ultraviolet Flood Exposure System}

The UV exposure OAI model (0130-040-03) consisted of three modules: power supply and intensity controller, a stand-alone light source and a shutter controller. The UV flood exposure provided the needed UV light at the specific $365 \mathrm{~nm}$ wavelength for processes such as the photolysis of the photobiotin and for the fabrication of silicon SU-8 master used for the fabrication of PDMS stamps required for the microcontact printing of proteins. The system is based on an ellipsoidal reflector and a set of lenses that focus the beam, producing an uniform illumination to an exposure area of approximately $133 \mathrm{~cm}^{2}$ (Figure A.8). The shutter controller allowed to control the time of exposure (values from 0.1-999 seconds) and the intensity controller helps to keep the intensity relatively constant throughout the lifetime of the lamp. The average intensity of the flood exposure is about $10 \mathrm{mw} / \mathrm{cm}^{2}$. 


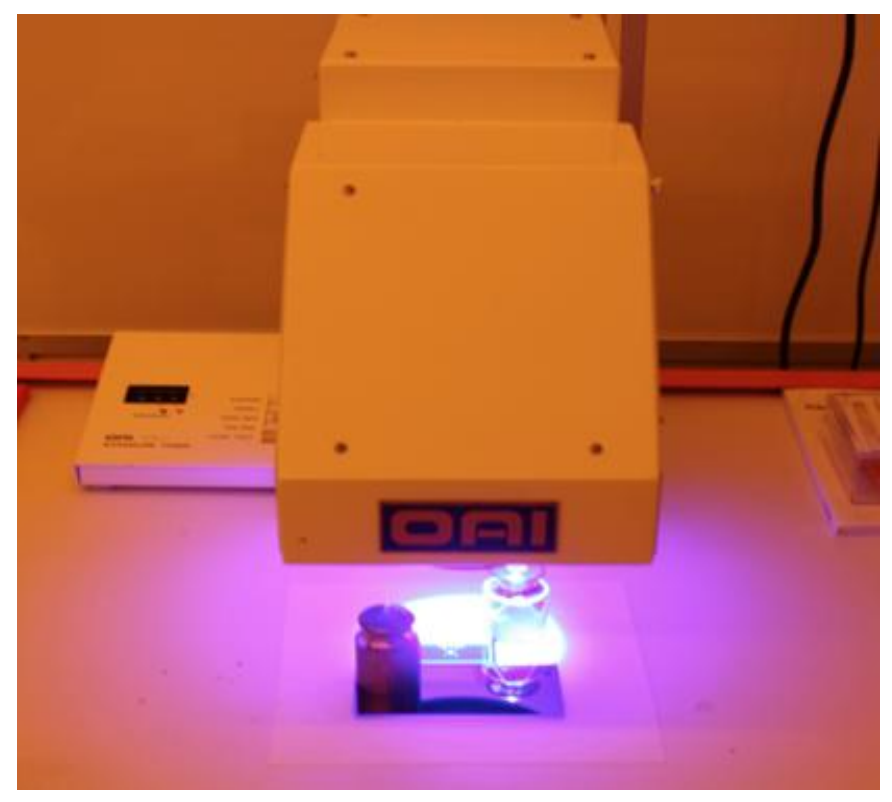

Figure A.8 Ultraviolet OAI 0130-040-03 flood exposure system

\section{A.3.2 Plasma Asher System}

The plasma cleaner was used to clean the surface of the PDMS stamps before incubation with the protein to improve the wettability of the surface.

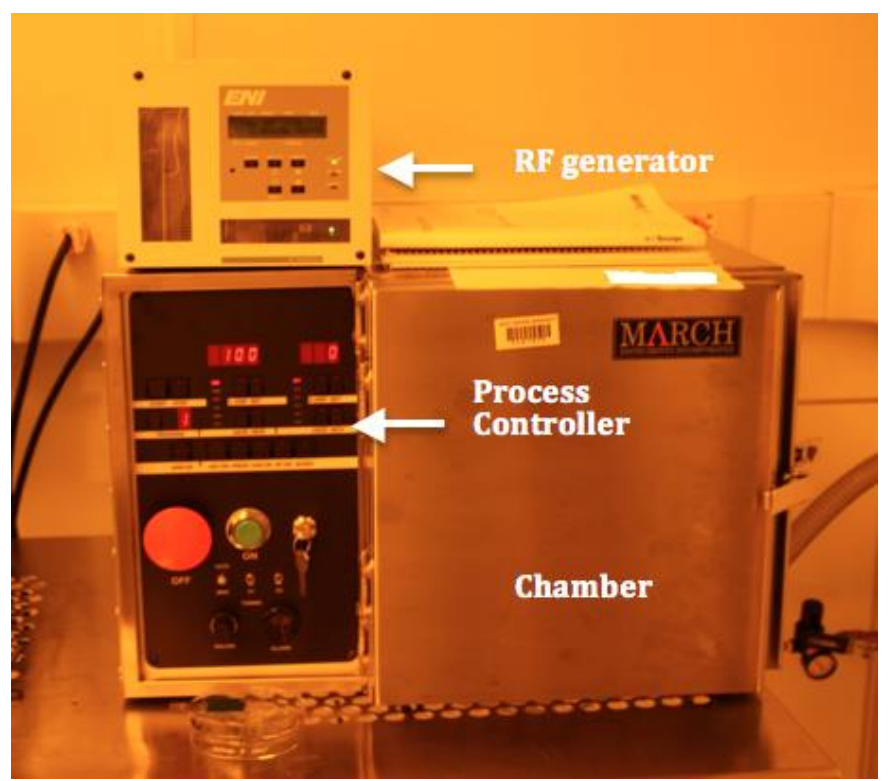

Figure A.9 Oxygen plasma cleaner system MARCH PX-250 
This instrument consists of three main modules (Figure A.9). The first module is the chamber where the reactions take place. The chamber dimension is around one cubit foot and has three trays where samples are placed. These trays can be swapped depending on the type of plasma operation to be used. The second module is the control module, and as the name suggests is used to set parameters of operation of the system. Parameters such as pressure, gas flow rate, exposition time, DC bias can be controlled depending on the process to be executed. The control module also has a programmable feature that allows users to save parameter values for a recurrent process. The third module is the radio frequency $(\mathrm{RF})$ generator, which consists of an oscillator that generates a signal at frequency $13.5 \mathrm{mHz}$, which is used to power and sustain the plasma during reactions.

This instrument is not only used for changing the wettability properties of the sample but also as an asher for total removal of materials or etching for selective removal. The purpose of the plasma asher cleaner was to change the surface properties of PDMS from hydrophobic (rejection of water) to hydrophilic (attraction of water) which contributes to the spreadibility of the protein on the surface of the PDMS during experiments. 


\section{APPENDIX B}

\section{B.1 Purification of Actin Filaments}

The actin proteins were supplied by Mr. Yongkuk Lee the other student in Dr Famouri's nanokinematics lab. The process of purification that Mr Lee used is described in this section as follows:

1. Mixing of Solutions of $1 \mathrm{M}$ of $\mathrm{NaHCO} 3$ and $3 \mathrm{M}$ of $\mathrm{KCl}$

2. Actin from rabbit muscle acetone powder is grounded 10minutes using mortar and pestle

3. The grounded powder is mixed with $5 \mathrm{ml}$ of $\mathrm{NaHCO} 3$ and stirred in ice for 10 minutes powder into $5 \mathrm{~mL} 1 \mathrm{M} \mathrm{NaHCO} 3$ then stir it well on ice for 10min. This step helps to separate G-Actin from the powder.

4. The solution is centrifuged at $4,000 \mathrm{rpm}$ for 10 minutes at $4{ }^{\circ} \mathrm{C}$

5. The supernatant is separated from the solution and placed in an empty conical tube and kept it on ice

6. The pellet is re-suspended in $5 \mathrm{~mL} \mathrm{NaHCO} 3$ and stirred in ice for another 10 minutes.

8. Repeat step 4 .

9. Repeat step 5

10. The supernatant is centrifuged twice, the first one is at $4000 \mathrm{rpm}$ and the second one is at 40000rpm, each of the centrifuge steps are done at $4^{\circ} \mathrm{C}$ for each minute each.

11. The supernatant is again separated and mixed in a ratio of 100:1 (v/v) with $3 \mathrm{M}$ of $\mathrm{KCL}$ to induce actin polymerization and incubate at $4^{\circ} \mathrm{C}$ for 12 hours.

12. After 12 hours Centrifuge the solution at 40,000rpm for 1.5 hours at $4^{\circ} \mathrm{C}$. 
13. Separate the supernatant and get the pellet (F-actin) and mix with M-buffer $(25 \mathrm{mM}$ $\mathrm{KCl}, 2 \mathrm{mM} \mathrm{MgCl} 2,0.2 \mathrm{mM} \mathrm{CaCl} 2$ and $25 \mathrm{mM}$ Imidazole at $\mathrm{pH} 7)$.

14. Dilute the original f-actin stock solution 4x, 10x 40x and 80x .

15. Determine the optical density of the diluted silutions using a spectrophotometer with a light wavelength of $290 \mathrm{~nm}$ and compare the obtained results with the reference value of $1 \mathrm{mg} / \mathrm{ml}$ of actin (which is 0.633 at the same light wavelength).

16. Make a final dilution of the solution of purified F-actin to a final concentration of $0.25 \mathrm{mg} / \mathrm{ml}$. 